\title{
Morphology and function of human spermatozoa
}

Citation for published version (APA):

Enginsu, M. E. (1994). Morphology and function of human spermatozoa. [Doctoral Thesis, Maastricht University]. Datawyse / Universitaire Pers Maastricht. https://doi.org/10.26481/dis.19940427me

Document status and date:

Published: 01/01/1994

DOI:

10.26481/dis.19940427me

Document Version:

Publisher's PDF, also known as Version of record

\section{Please check the document version of this publication:}

- A submitted manuscript is the version of the article upon submission and before peer-review. There can be important differences between the submitted version and the official published version of record.

People interested in the research are advised to contact the author for the final version of the publication, or visit the DOI to the publisher's website.

- The final author version and the galley proof are versions of the publication after peer review.

- The final published version features the final layout of the paper including the volume, issue and page numbers.

Link to publication

\footnotetext{
General rights rights.

- You may freely distribute the URL identifying the publication in the public portal. please follow below link for the End User Agreement:

www.umlib.nl/taverne-license

Take down policy

If you believe that this document breaches copyright please contact us at:

repository@maastrichtuniversity.nl

providing details and we will investigate your claim.
}

Copyright and moral rights for the publications made accessible in the public portal are retained by the authors and/or other copyright owners and it is a condition of accessing publications that users recognise and abide by the legal requirements associated with these

- Users may download and print one copy of any publication from the public portal for the purpose of private study or research.

- You may not further distribute the material or use it for any profit-making activity or commercial gain

If the publication is distributed under the terms of Article $25 \mathrm{fa}$ of the Dutch Copyright Act, indicated by the "Taverne" license above, 


\section{Morphology and function of human spermatozoa}


Cover Design: Peter Fransman, Engin Enginsu

CIP-DATA KONIKLIJKE BIBLIOTHEEK, DEN HAAG

Enginsu, Mehmet Engin

Morphology and function of human spermatozoa / Mehmet

Engin Enginsu. - Maastricht: Universitaire Pers

Maastricht. - III.

Thesis Maastricht. - With ref. - With summary in Dutch and Turkish.

ISBN 90-5278-133-8

Subject headings: sperm morphology / male infertility. 


\section{Morphology and function of human spermatozoa}

\section{PROEFSCHRIFT}

ter verkrijging van de graad van doctor

aan de Rijksuniversiteit Limburg te Maastricht, op gezag van de Rector Magnificus, Prof.dr H. Philipsen, volgens het besluit van het College van Dekanen, in het openbaar te verdedigen op woensdag, 27 april 1994 om 16.00 uur

door

Mehmet Engin Enginsu

geboren op 4 december 1962 te İzmir, Turkije 


\section{Promotores:}

Prof. Dr. J.L.H. Evers

Prof. Dr. J.P.M. Geraedts

Beoordelingscommissie:

Prof. Dr. R.A. Janknegt (voorzitter)

Prof. Dr. H.F.J.M. Crebolder

Prof. Dr. M.P. van Dieijen-Visser

Prof. Dr. S. Gordts (Université Catholic de Louvain, België)

Prof. Dr. R. Rolland (Katholieke Universiteit Nijmegen) 
This thesis is dedicated to my parents, Yüksel and Ayla, and to my sister Pinar.

For your constant support and love. 
$\begin{array}{lr}\text { Abbreviations } & 9\end{array}$

Chapter I

General Introduction 11

1. Introduction 11

2. Spermatogenesis 13

2.1. Spermatogonia development 13

2.2. Spermatocyte development 16

2.3. Spermiogenesis 16

3. Sperm Function 18

3.1. Maturation 18

3.2. Capacitation 19

3.3. Acrosome Reaction 20

3.4. Fertilization 22

4. Diagnosis of Sperm Fertilizing Ability 25

4.1. Basic Semen Analysis 25

4.2. Biochemical Tests 26

4.3. Immunological Tests 27

4.4. Functional Tests 28

5. Statistical Analysis 29

6. Aims of this study 30

Chapter II

Evaluation of human sperm morphology using strict criteria after Diff-Quik staning: correlation of morphology with fertilization in vitro

Chapter III

Comparison between the hypoosmotic swelling test and morphology evaluation using strict criteria in predicting in vitro fertilization (IVF)

Chapter IV

Male factor as a determinant of in vitro fertilization outcome 
Predictive value of morphologically normal sperm concentration in the medium for in vitro fertilization

\section{Chapter VI}

The effect of pentoxifylline on intracellular sperm cAMP concentration

Chapter VII

General Discussion

Summary / Samenvatting / Özet

Appendix 1

Appendix 2

Thank You

Curriculum Vitae

List of Publications 
AMF Anti Müllerian Factor

ART Assisted Reproductive Technologies

ASA Anti-sperm Antibody

ATP Adenosine triphosphate

CASA Computer Assisted Semen Analysis

cAMP Cyclic Adenosine

Monophosphate

CI Confidence Interval

CK Creatin Kinase

DAG Diacylgyserol

DHT Dihydro testosterone

DNA Deoxyribonucleic acid

ET Embryo Transfer

FITS Fluorescein isothiocyanate

FSH Follicle Stimulating

Hormone

GPO Gepasteuriseerde Plasmaeiwit Oplossing (human serum protein solution)

GTP Guanosine 5'-triphosphate

hCG Human chorionic gonadotrophin

hMG Human menopausal gonadotrophin

HOST Hypoosmotic Swelling Test

HTF Human Tubal Fluid

HZA Hemizona Assay

ICSI Intra Cytoplasmic Sperm

Injection

IMNS Inseminated

Morphologically Normal

Spermatozoa

IBT Immunobead-reaction Test

IVF In Vitro Fertilization

IVO Volume of IVF medium

LH Luteinizing Hormone

LR Likelihood Ratio
LUF Luteinized Unruptured Follicle

MAR Mixed Antiglobulin Reaction (test)

MEUSC Morphology Evaluation Using Strict Criteria

OA Observed Agreement

AC Agreement by Chance

PCT Post-coital Test

PF Pentoxifylline

PMSD Progressive Motile Sperm Density

POF Premature Ovarian Failure

ROC Receiver Operating

Characteristics

SCSU Sperm concentration of the swim up fraction

SPA Sperm Penetration Assay (also known as hamster oocyte/sperm penetration assay)

SPERM Subfertile patient evaluation regarding morphology

SRY Sex determining region of the $\mathrm{Y}$ chromosome

TDF Testis Determining Factor WHO World Health Organization ZFY Zinc finger gene protein on the $\mathrm{Y}$ chromosome 


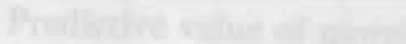

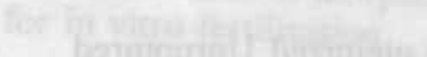

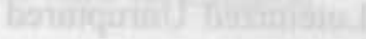

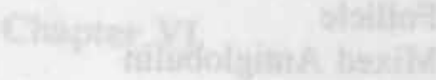

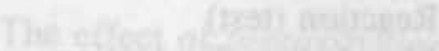

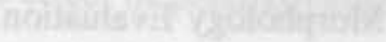

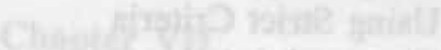

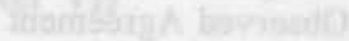

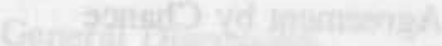

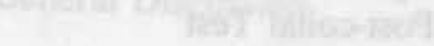

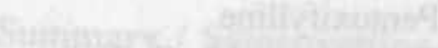

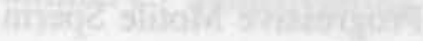

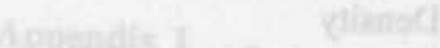

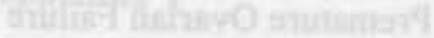

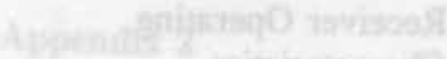

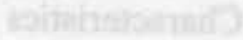

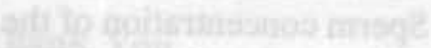

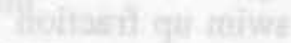

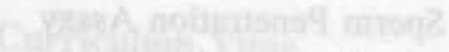

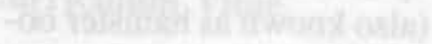

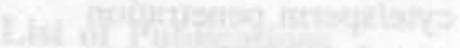
(17aly

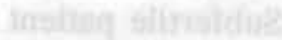

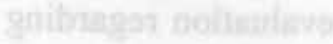

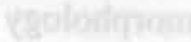

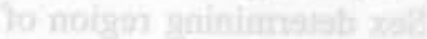

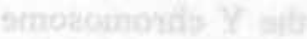

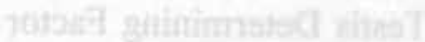

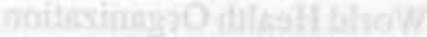

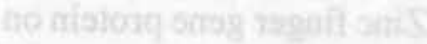

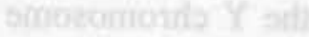

914:

MAN

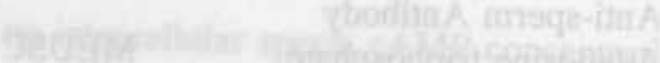

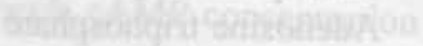

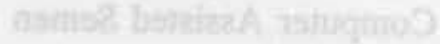
gizvina $A$ aniconobA shibio

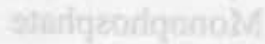
Lovionin sonabilino?

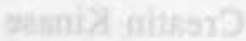
inseyglyaid sucadianat oxbufica

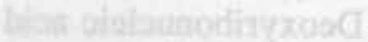
vatenthT ovadimu U20 A97?

M519Me

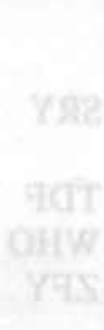

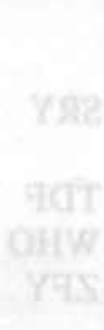

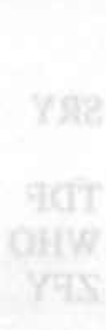

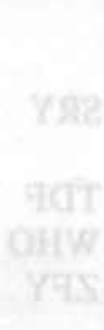
707 909

avisubonage basiaca sipolonitod yrisingate sfillo' anoumbia

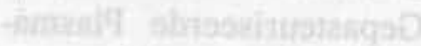

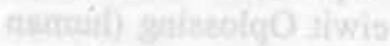

Gavilution wisiong mimot

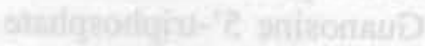

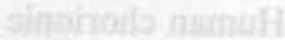
Ailinconobanog Lemeganam ngrnoli

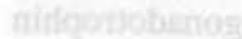

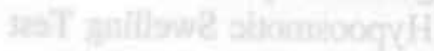

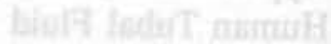
itacth puosimall Tgh 8 9TR APAOT GHAS 14:5870 DAC lififici AWICI 117) 2719 121492

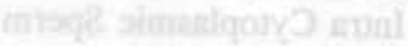
axymagialy

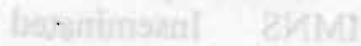
17098 126 970 1004

Divat

72013 77. H A.SH 1201

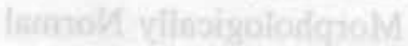

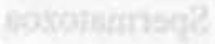

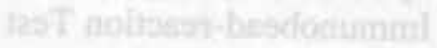

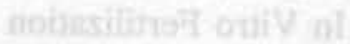
maibon 7V/1 Jo samulo?

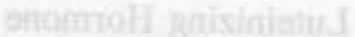
oidegr boondalsy.]
787 जुत 0VI 1月. 1 9.1 


\section{General Introduction}

\section{Introduction}

The World Health Organization (WHO) defines infertility as lack of conception after at least twelve months of unprotected intercourse. Primary male infertility means that the man has never impregnated a woman. In case of secondary male infertility the man has impregnated a woman before, irrespective of the outcome of the pregnancy, but is not being able to do so now (97).

In a study done by the WHO, 7273 couples were evaluated in respect to their cause of infertility (23).

Table I. Sex distribution of causes of infertility, WHO (97).

CAUSES OF INFERTILITY

Female factor only

Male factor only

Female and Male factors

No demonstrable cause
PERCENTAGE

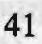

24

24

11

It can be seen from Table I that in $48 \%$ of the couples suffering from infertility, a male factor is involved. The causes of male infertility are also shown in Table II.

Some studies suggest that during recent decennia infertility is on the increase. Especially male fertility has been suggested to be declining. Two recent studies give more insight into this increase with respect to the male partner:

Ginsburg and Hardiman have studied the partners of women undergoing gonadotrophin stimulant therapy in two six year periods (1977-83 and 1983-89) (37). A significant decrease was detected in sperm density from 101 million per $\mathrm{ml}$ to 96 million per $\mathrm{ml}$. The proportion of men with low sperm density ( $<40$ million per $\mathrm{ml}$ ) increased significantly from $5.8 \%$ to $16.3 \%$, with decreased sperm motility $(<50 \%$ ) increased significantly from $20.7 \%$ to $34.4 \%$, and with decreased morphology ( $<50 \%$ normal forms) increased significantly, from $1 \%$ to $12.3 \%$ (37).

Secondly, a literature review from Carlsen and coworkers, has focused on the quality of semen during the period of 1939-1989 (16). The study included 61 papers and 14947 men. Their results show a significant decrease of the seminal volume from $3.4 \mathrm{ml}$ to $2.75 \mathrm{ml}$ and a significant decrease of the sperm density from 113 million per $\mathrm{ml}$ to 66 million per $\mathrm{ml}$. Since the evaluation of motility and morphology were not always available in the older publications, sperm density was used as an indicator for semen quality. They have also indicated 
that during the same period the incidence of some genitourinary abnormalities including testicular cancer, maldescent and hypospadias had increased. However, above mentioned study has been criticized by other authors $(12,38$, 93).

Table II. Distribution of diagnosis of male infertility (23).

\begin{tabular}{lrr}
\hline Diagnosis & Number of patients & Percentage \\
\hline No demonstrable cause & 3127 & 48.8 \\
Varicocele & 806 & 12.6 \\
Idiopathic oligozoospermia & 717 & 11.2 \\
Accessory gland infection & 441 & 6.9 \\
Idiopathic teratozoospermia & 376 & 5.9 \\
Idiopathic asthenozoospermia & 252 & 3.9 \\
Isolated seminal plasma abnormalities & 224 & 3.5 \\
Suspected immunological infertility & 193 & 3.0 \\
Congenital abnormalities & 106 & 1.7 \\
Systemic diseases & 91 & 1.4 \\
Sexual inadequacy & 81 & 1.3 \\
Obstructive azoospermia & 58 & 0.9 \\
Idiopathic necrozoospermia & 49 & 0.8 \\
Ejaculatory inadequacy & 42 & 0.7 \\
Hyperprolactinemia & 39 & 0.6 \\
Iatrogenic causes & 36 & 0.5 \\
Karyotype abnormalities & 31 & 0.1 \\
Partial obstruction & 6 & 0.1 \\
Retrograde ejaculation & 4 & 0.1 \\
Immotile cilia syndrome & 1 & $<0.1$ \\
Pituitary lesions & 1 & $<0.1$ \\
Gonadotrophin deficiency & 1 & $<0.1$ \\
\hline & &
\end{tabular}

Tummon and Mortimer (93) did draw attention to the methodological differences between the early and the late papers. Furthermore, they questioned the quality of the semen analysis performed more than 30 years ago, and concluded that the comparison between the historical and the contemporary methods may not be reliable since semen evaluation techniques have improved dramatically in the last decade allowing a more accurate evaluation than before. Brake and Krause (12) criticized Carlsen's study for the statistical methods used for the evaluation of the literature. They believe that linear regression analysis is improper to evaluate the data of Carlsen article. Therefore, the results should not be interpreted in such a way that the decline in the sperm quality has a linear character. Ginsburg and Hardiman (38) did also criticize the methodology of Carlsen's article, although they confirmed the trend in the declining sperm quality. Although Carlsen, and coworkers replied the criticisms (17), the reply contained no convincing evidence that their method of evaluation was correct. However, when both Carlsen and Ginsburg and Hardiman studies are evaluated, 
the declining semen quality which is in parallel with an increasing percentage of genitourinary abnormalities, should be considered seriously and further studies should be conducted to enlighten the cause of the decline.

The possible decline in semen quality has led to many speculations regarding the nature. During the last decade many potential risks have been studied: metals (lead, cadmium), synthetic hormones (environmental estrogens), chemicals (insecticides, pesticides, herbicides) and solvents (especially ethylene glycols). Studies in specific professional categories have been undertaken in fire fighters, welders, oil refinery workers, farmers and dry cleaning workers (87). The people who are exposed to the above mentioned substances or the people who are in the above mentioned professions are considered to be in the risk group for male subfertility. More detailed and specific information concerning the individual effects of these substances and occupations can be found in some other studies $(20,34,42,102)$. Briefly; chronic exposure to the above mentioned substances may reduce fertility and excessively hot environments may depress spermatogenesis (97), and since the germinal epithelium is one of the most proliferative tissues of our body it reacts with particular sensitivity to changes of the environment. The studies detecting, the possible toxic factor(s) and their role on human sperm quality, should therefore be encouraged.

After the introduction of Assisted Reproductive Technologies (ART) awareness of infertility as a treatable medical problem has increased. This has been accomplished by dramatic rise in the number of visits to infertility specialists. In Vitro Fertilization (IVF) was first designed to overcome female problems (88). Significant progress in male reproductive biology and our knowledge of human fertilization process have provided the basis for treatment of many male reproductive failure cases (105). More recently, the diagnostic properties of ART as a test to detect fertilization failure as have been appreciated (57). Also with improved methods of sperm preparations, it became apparent that ART could also be used for the treatment of subfertile men.

This introduction will further focus on the different stages of sperm maturation, sperm function and diagnosis of sperm function.

\section{Spermatogenesis}

\subsection{Spermatogonia Development}

Spermatogenesis is a process that serves to expand the number of germ cells and to permit their development into mature spermatozoa. This process takes place in the seminiferous tubule, a long tubular structure lined by a single layer of Sertoli cells.

Initially, the undifferentiated gonad consists of mesoderm that will, in the male eventually differentiate into the somatic elements of the testis. Differentiation of the bipotential gonad in the male direction is determined by the presence and 
expression of a DNA sequence on the Y chromosome. In humans this sequence is referred to as the Testis Determining Factor (TDF). The search for the TDF has been both exciting and frustrating (63). In the time period from 1959 till 1990 the localization of TDF was narrowed down from the whole Y chromosome to less than 250 bases encoding the conserved 80 -amino-acid sequence of the sex determining region of the Y (SRY)(Figure 1). Originally it was thought that TDF was the zinc finger gene protein (ZFY) which was isolated by Page and coworkers (75). However, ZFY fell out off favor as the "testis determining factor" after three XX men and one XX intersex who lacked ZFY were found. Furthermore, expression of the ZFY homologue in mice proved to be associated with the germ cells, which are believed to play no part in testis determination. Subsequently, the SRY gene was isolated on the human Y chromosome (85). In a recent review, Mittwoch gives more insight and detailed information on sex determination and concludes that SRY gene functions during the initial stages of testicular differentiation, whereas other $\mathrm{Y}$ chromosomal genes are required to maintain the germ cells and allow their proliferation and differentiation (68). The process leading to the development of the indifferent fetal gonads into testes or ovaries is described as "primary sex differentiation" whereas the development of the Müllerian and Wolffian duct systems is called "secondary sex differentiation" (31). In the male two hormones from the fetal testes are responsible for directing the appropriate differentiation of the Müllerian ducts and Wolffian ducts and urogenital sinus; Sertoli cells produce a protein called anti Müllerian factor (AMF) which causes the regression of Müllerian ducts (51). Leydig cells produce testosterone, which stimulates growth and differentiation of the urogenital sinus into the peripheral zone of the prostate while stimulating the Wolffian ducts to develop into the central zone of the prostate as well as the epididymis, vas deferens, and seminal vesicle $(15,64,98)$.

Leydig cells are the interstitial cells of the testis. They begin to differentiate from interstitial mesenchyme in the 8 th week of human embryonic development. Shortly thereafter the population becomes competent as steroid-secreting tissue, with embryonal peak production of testosterone in the 12th week. This has important effects such as; promoting differentiation of epididymis and vas deferens and stimulating differentiation of the accessory glands. Testosterone is converted to dihydro testosterone (DHT) with $5-\alpha$-reductase. This conversion is crucial for the differentiation of external genitalia since the patients with $5-\alpha$ reductase deficiency have normally virilized internal genitalia but predominantly female external genitalia (99).

During the 5 th month of development, there is rapid disappearance of recognizable Leydig cells, with testosterone reaching its lowest levels in embryonic life by the 6 th month. Leydig cells regain activity at the time of puberty when gametogenesis starts.

During spermatogenesis, relatively undifferentiated spermatogonia slowly evolve into highly specialized testicular spermatozoa. Several generations of cells may 
always be seen together in the seminiferous epithelium. The youngest generation, which consists of spermatogonia just beginning differentiation, is found at the periphery at the tubule, while the oldest generation, which consists of spermatids, lines the lumen.

Figure 1. The search of testis determining factor (TDF) in 31 years (63).

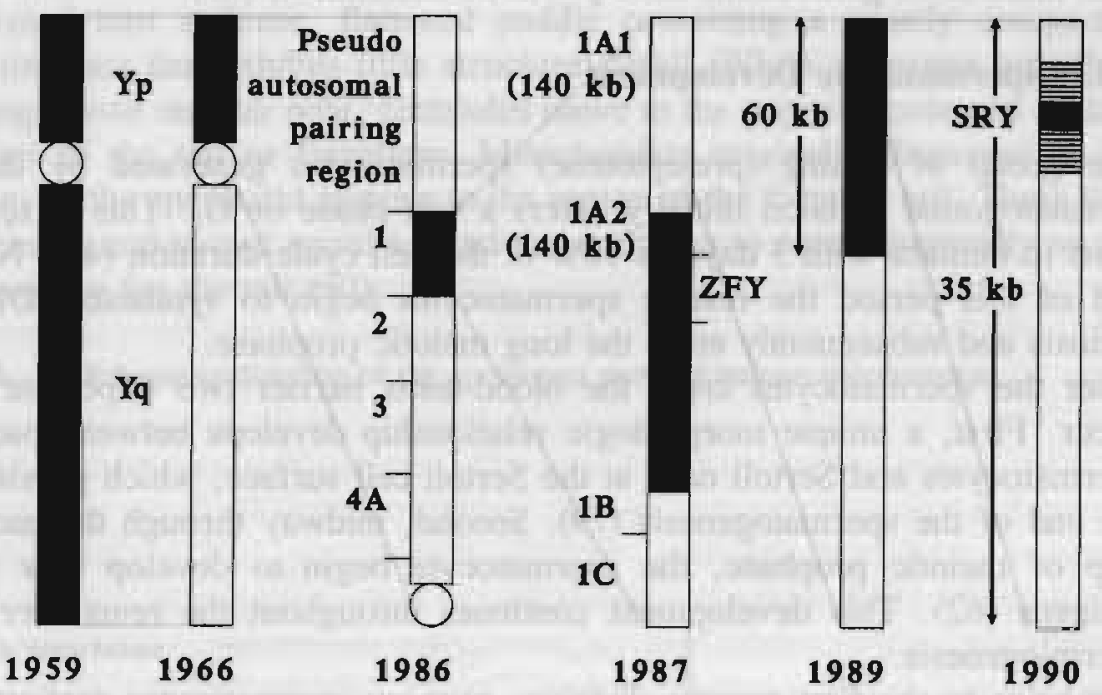

During spermatogenesis the actual number of stages of maturation and their boundaries vary from species to species. Separate stages have been based on the development of the acrosomal cap ( 14 have been described in the rat, 12 for the mouse and the monkey, and 6 for the human). The successive appearance of all stages over time constitutes the cycle of the seminiferous epithelium.

The process of spermatogenesis can be subdivided into four successive components, each of which has a set of unique characteristics and requirements:

1. Undifferentiated spermatogonial renewal and proliferation

2. Spermatogonial differentiation

3. Spermatocyte development, meiosis

4. Spermatid development, spermiogenesis

The first two stages are poorly defined in the human. However, much knowledge has been gathered from the studies of the rodent testis $(19,65)$.

In the human testis following spermatogonial types have been recognized; $\mathrm{A}_{\text {long, }}$, $A_{\text {dark }}, A_{\text {pale }}, B$. Additional types of $A$ cells have recently been recognized. TypeB spermatogonia are differentiated cells that ultimately produce resting 
(preleptotene) spermatocytes. All type A spermatogonia have been considered for a stem cell role. In a variety of testicular pathologies and experimental conditions, such as azoospermia, cryptorchidism, Klinefelter's syndrome, hypogonadotrophic hypogonadism, anti-androgen treatment, estrogen treatment and irradiation chemotherapy $A_{\text {pale, }}$, and not $A_{\text {dark }}$ spermatogonia persist. Recent studies suggest that morphologic changes are associated with the cell cycle and/or the presence of additional generations. In humans, either the various types of the spermatogonia have widely disparate life cycles or, more likely, the total number of generations have yet to be determined (89).

\subsection{Spermatocyte Development}

The group of resting (preleptotene) spermatocytes generated by the final spermatogonial division initially enters a rest phase or $G_{1}$. This is relatively short in humans with 3 days or $16 \%$ of the cell cycle duration (41). Near the end of this period the resting spermatocytes begin to synthesize DNA for meiosis and subsequently enter the long meiotic prophase.

After the spermatocytes cross the blood-testis barrier two important events occur. First, a unique morphologic relationship develops between pachytene spermatocytes and Sertoli cells at the Sertoli cell surface, which persists until the end of the spermatogenesis (79). Second, midway through the pachytene step of meiotic prophase, the spermatocyte begin to develop new surface antigens (62). This development continues throughout the remainder of the spermiogenesis.

Just prior to the first meiotic division, primary spermatocytes replicate their DNA. Each of the 46 chromosomes is visible as a double structure. After the first meiotic division, each daughter cell (secondary spermatocyte) contains onehalf the normal number of chromosomes. Since each chromosome is a double structure, the total amount of DNA in each daughter spermatocyte is equal to that of a normal somatic cell in the $G_{1}$ phase. During the second meiotic division, each double structured chromosome divides at the centromere longitudinally and each daughter cell (spermatid) receives 23 chromosomes. Since no DNA synthesis takes place before this division, each spermatid contains one-half the number of chromosomes, and one-half the amount of DNA of a normal somatic cell in the $G_{0}$ and $G_{1}$ phase (80).

\subsection{Spermiogenesis}

Spermiogenesis is the stage where the spermatids mature into spermatozoa. The most obvious event is the development of the acrosome. In the initial phase of the process, the Golgi apparatus generates material for the acrosome, which develops like a tight fitting cap, partially covering the head of the nucleus. The acrosome actually is a modified lysosomal bag containing a number of enzymes 
rich in glycoproteins (see below) which are instrumental in dissolving a path through the zona pellucida of the oocyte at the time of fertilization.

Early in spermiogenesis, while the acrosome is growing, the spermatid nucleus remains a metabolically active spherical body. The nucleus migrates to the periphery of the cell. Then the cap is fully formed and the acrosome becomes intimately invested by the overlying plasma membrane (Figure 2$)(18,33)$.

Completion of this migration signals the gradual cessation of nuclear metabolic activity, the onset of chromatin condensation, and the beginning of the nuclear elongation. At the completion of spermiogenesis, the nucleus has been transformed into a dense, flattened paddle containing a greatly compacted chromatin mass that exhibits little structural detail. While acrosome growth is occurring at one nuclear pole, centrioles move to the opposite pole and initiate formation of the tail or flagellum. Mitochondria originally dispersed at the periphery of the spermatid migrate to the region of the forming tail. There they line up in an end-to-end spiral around the midpiece to serve eventually as the energy source for the tail (50).

Figure 2. Schematic drawing of the acrosomal parts of human spermatozoa.

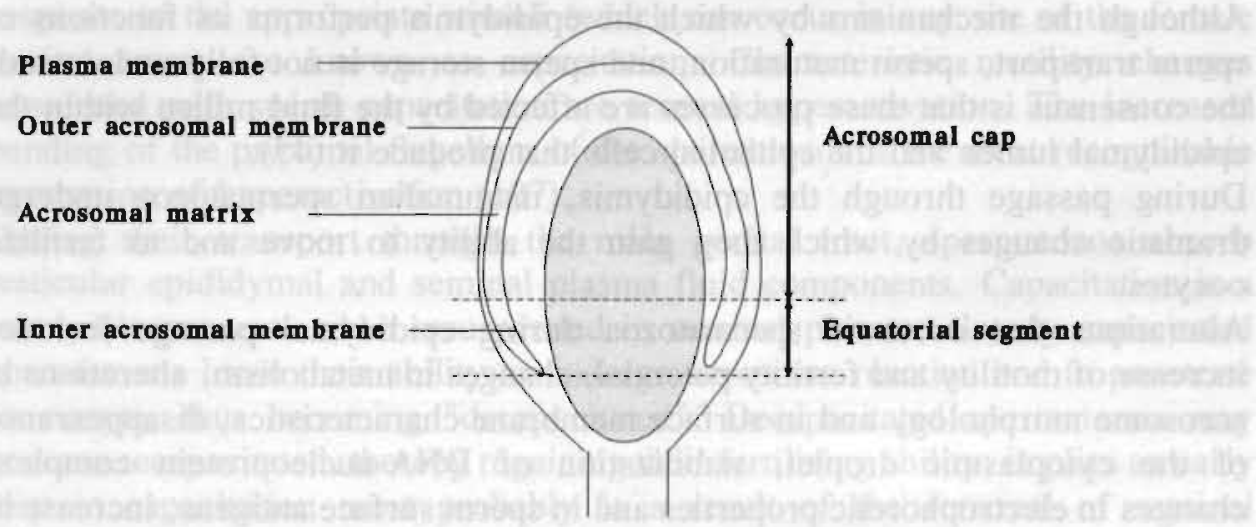

Extensive ectoplasmic events take place between the spermatids and the Sertoli cells over the region of the acrosomal cap as spermatids begin to elongate. These events are thought to be instrumental in the orderly migration of germ cells through the lumen and in the maintenance of intimate physical contact between germ cells and Sertoli cells. It appears that these specializations are pivotal in determining the actual time of sperm release. The released spermatozoa are neither motile nor able to fertilize; such properties are acquired during 
their passage through the epididymis.

During the spermiogenesis, most of the cytoplasm, including the intracellular bridges and remaining organelles, are shed as a "residual body" which is immediately phagocytized by Sertoli cells (72).

Especially in the terminal phase of spermiogenesis, there is extensive evidence for hormonal requirement. However, since there is no evidence of active hormone receptors on the spermatids themselves, hormonal requirements are likely fulfilled indirectly through the Sertoli cells, which are known targets for both FSH and testosterone (86).

\section{Sperm Function}

\subsection{Maturation}

The maturation of the spermatozoa continues in the epididymis.

The epididymis consists of the ductuli efferentes and the ductus epididymidis. Between 10 and 15 ductuli efferentes arise from the rete testis. These tubules come together to form an epididymal duct which vary between 3 to 4 meters in man. The epididymis is conventionally divided into three anatomic segments: the head (caput), body (corpus), and tail (cauda). The caput epididymidis is involved in sperm maturation and the cauda epididymidis is the area of sperm storage.

Although the mechanisms by which the epididymis performs its functions of sperm transport, sperm maturation, and sperm storage is not fully understood, the consensus is that these processes are affected by the fluid milieu within the epididymal lumen and the epithelial cells that produce it (45).

During passage through the epididymis, mammalian spermatozoa undergo dramatic changes by which they gain the ability to move and to fertilize oocytes.

Alterations that occur in spermatozoa during epididymal passage include: increase of motility and fertility potential, changes in metabolism, alterations in acrosome morphology and in surface membrane characteristics, disappearance of the cytoplasmic droplet, stabilization of DNA-nucleoprotein complex, changes in electrophoretic properties and in sperm surface antigens, increase in the number of disulfide bonds, alterations in surface-coating glycoproteins, increase of cAMP content. It is not possible to screen epididymal sperm maturation directly in man. Therefore spermatozoa collected from different parts of the epididymis were subjected to motility evaluation and sperm penetration assay (hamster ovum test). The results give strong evidence that human spermatozoa complete normal maturation in mid epididymis (104). The studies from Brooks and Cohen and coworkers $(13,21)$ indicate that motility and fertility of the epididymal spermatozoa are androgen-dependent processes.

Transport of the spermatozoa through the epididymis occurs partly by the 
muscle tissue surrounding the tubules and partly by the ciliated cells within tubules (49). The major storage site of the spermatozoa within the excurent duct system is the cauda epididymidis. Depending on the ejaculatory frequency of the individual, sperm storage time is in the range of 3-10 days (5). In men 2130 year of age, the stored sperm in each epididymis is approximately 200 million, with more than half of the spermatozoa present in the cauda epididymidis. In men around 45 years of age the number decreases to 150 million (about $50 \%$ in the cauda).

Silber and coworkers, studied the fertilizing capacity of human sperm, collected from epididymis in a series of $28 \mathrm{men}$, who were suffering from infertility due to the congenital absence of the vas deferens (84). Spermatozoa from different parts of epididymis were obtained by using microsurgical techniques. Evaluation of the fertilizing ability of these spermatozoa showed strong evidence that sperm do not require transit through the epididymis in order to fertilize an oocyte under in vitro conditions. Although, in a later study, they have stated this phenomenon also after evaluating the pregnancy outcome in such cases, the results in both studies do not rule out the possibility, that retrograde epididymal secretions may still support or be necessary for sperm maturation.

\subsection{Capacitation}

The modifications that are required for the acrosome reaction to occur in response to the appropriate stimuli (which can occur in any site of the female genital tract) are known as capacitation. Characteristic motility changes associated with sperm capacitation are termed hyperactivation. The increased bending of the proximal flagellum of the spermatozoa is the most recognizable expression of hyperactivation (107).

During their transport through the male genital tract, spermatozoa absorb testicular epididymal and seminal plasma fluid components. Capacitation is a reversible process: when resuspended in seminal plasma, already capacitated spermatozoa loose their ability to undergo acrosome reaction and to penetrate an oocyte, thus becoming "decapacitated". Decapacitated spermatozoa may become recapacitated, thereby regaining their fertilizing ability; implies an early step in capacitation most probably is a result of the removal of seminal inhibitory factors.

Among the many epididymal and seminal plasma factors which adhere to the spermatozoa, three are known to prevent fertilization in a reversible manner. First there is a group of high molecular weight glycoproteins. These glycoproteins differ in the mechanism by which they counteract the fertilizing capacity of spermatozoa. One prevents the acrosome reaction whereas another prevents zona penetration without an apparent effect on the acrosome reaction. Secondly, there is acrostatin, which inhibits both proacrosin and acrosin (two acrosomal enzymes which play an important role in acrosome reaction). Finally high 
molecular weight polyactosaminyl glycoside is found, which is a glycosyltransferase inhibitor. "Decapacitation factors" is the commonly term to summarize the above mentioned factors.

The cholesterol/phospholipid ratio of the human sperm plasma membrane is important for membrane stability. A decrease in cholesterol or an increase in phospholipid content causes destabilization of the membrane and an increase in membrane permeability (59).

Cholesterol loss from the membranes usually occurs via carrier proteins or enzymes which can be found in the female genital secretions $(28,59)$. These are also essential components of the media used for in vitro capacitation and fertilization.

In the testis, the activity of the cholesteryl ester hydrolases is regulated by proteinkinase A. This hydrolase has been implicated in controlling the sperm membrane cholesterol level (8). Therefore, sperm proteinkinase A may function to regulate sperm membrane cholesterol in ejaculated spermatozoa as well.

Capacitation has also been shown to be dependent on the composition of the environment. Certain levels of free calcium, sodium and potassium, and energy substrates are required for capacitation to occur (36).

The time it takes to complete capacitation can be estimated by incubating spermatozoa in artificial media. Minimum in vitro capacitation time for human spermatozoa has been reported to vary between 4 to 6 hours (77).

\subsection{Acrosome Reaction}

The acrosome is a membrane-bound, cap-like structure covering the anterior part of the sperm head (7). The acrosome can be divided into three parts: the outer acrosomal membrane, the acrosomal matrix, and the inner acrosomal membrane. The inner and outer acrosomal membrane join at the equatorial segment. The entire spermatozoon including the acrosome, is surrounded by a plasma membrane (Figure 2).

The acrosome reaction, consists of a series of alterations of the acrosome leading to its eventual complete loss (Figure 3). Although capacitation is a prerequisite for the acrosome reaction, there is no clear demarcation between the two reactions. Capacitation is reversible and involves changes at the biochemical level; the acrosome reaction is irreversible and is morphologically visible as loss of the acrosome. The composition of the outer acrosomal membrane and the plasma membrane can easily be altered, since these membranes are relatively unstable. In the moribund and/or dead spermatozoa degenerative acrosomal modifications occur. Bedford proposed to call these "false acrosome reaction" as opposed to the true acrosome reaction which can only occur in the viable spermatozoa (10).

The acrosome contains many different hydrolytic enzymes (Table III) (103). Acrosin which is found as proacrosin (the inactive form of acrosin) and 
hyaluronidase are among the most extensively studied and best characterized enzymes. The female genital tract possesses a mechanism to prevent the activation and release of these enzymes until the spermatozoon reaches the zona pellucida which is the target. Initiation of the acrosome reaction is most likely triggered by the receptors on the plasma membrane. A zona glycoprotein ZP3, induces acrosome reaction. However, before reaching to the target site, spermatozoa exposed to other factors e.g. progesterone, prostaglandins, sterol sulphatase, glycoaminoglycans, which are present in the follicular fluid and the cumulus cell secretions (such as hyaluronidase), can also induce acrosome reaction.

Ion transports also govern acrosome reaction and are primarily controlled by enzymes and channel proteins. The influx of $\mathrm{Ca}^{+2}$ can modulate the acrosome reaction, but it is not the initiating factor. Furthermore, stimulation of GTP binding proteins (G-proteins) (6) or proteinkinase A (30) induces acrosome reaction in the absence of $\mathrm{Ca}^{+2}$ from the medium. Different ATPases are associated with the plasma membrane and the outer acrosomal membrane, so that the ionic composition of the cytosolic and intra acrosomal spaces may vary. Briefly $\mathrm{Na}^{+}-\mathrm{K}^{+}$ATPase and $\mathrm{Ca}^{2+}$ ATPase pump $\mathrm{Na}^{+}$and $\mathrm{Ca}^{2+}$ out of the cell respectively, and $\mathrm{Mg}^{2+}$ ATPase may maintain high intracellular $\mathrm{H}^{+}$levels.

During acrosome reaction these pumps are either overwhelmed or inhibited: $\mathrm{Na}^{+}$and $\mathrm{Ca}^{2+}$ influx and $\mathrm{H}^{+}$efflux occur. The change of $\mathrm{pH}$ may cause activation of proacrosin to acrosin.

At least two second messenger systems are operative in the acrosome reaction $(29,30)$. First, adenylate cyclase is activated and formation of cAMP results. cAMP activates proteinkinase A and causes protein phosphorylation. Secondly, formation of diacylglycerol (DAG) and inositol triphosphate results from the activation of a phospholipase $\mathrm{C}$ which hydrolyses phosphatidylinositol biphosphate $\left(\mathrm{PIP}_{2}\right)$. Both of these pathways play an important role in acrosome reaction.

Table III. Enzymes of acrosomal origin.

$\begin{array}{ll}\alpha \text {-L-Fucosidase } & \text { Acid proteinase } \\ \text { Acrosin } & \text { Aryamidase } \\ \text { Arylsulphatase } & \text { Cathepsin-D } \\ \text { B-Galactosidase } & \text { B-Glucronidase } \\ \text { B-N-Acetylglucosaminidase } & \text { Esterase } \\ \text { B-N-Acetylhexosaminidase } & \text { Collagenase } \\ \text { Hyaluronidase } & \text { Neuroaminidase } \\ \text { Ornithine decarboxylase } & \text { Peptidyl peptidase } \\ \text { Phosphatase } & \text { Phospholipase } \\ \text { Phospholipase-C } & \end{array}$

Nagae and coworkers have proposed the following morphological sequence for the acrosome reaction (71): 
1 The anterior part of the acrosomal cap swells due to decondensation of the acrosomal matrix.

2 The outer acrosomal membrane-alone, or with the plasma membraneinvaginates to form vesicles.

3 Fusion occurs between the plasma membrane and the outer acrosomal membrane. Fusion in the acrosomal cap is rarely seen, but fusion is consistently seen at the anterior part end of the equatorial segment.

4 The plasma membrane and the outer acrosomal membrane of the acrosomal cap region disappear, leaving many vesicles on or near the inner acrosomal membrane.

5 These vesicles disperse, leaving the spermatozoon surrounded by the single, denuded inner acrosomal membrane.

Figure 3. Schematic drawing of acrosome reaction in human spermatozoa (71).
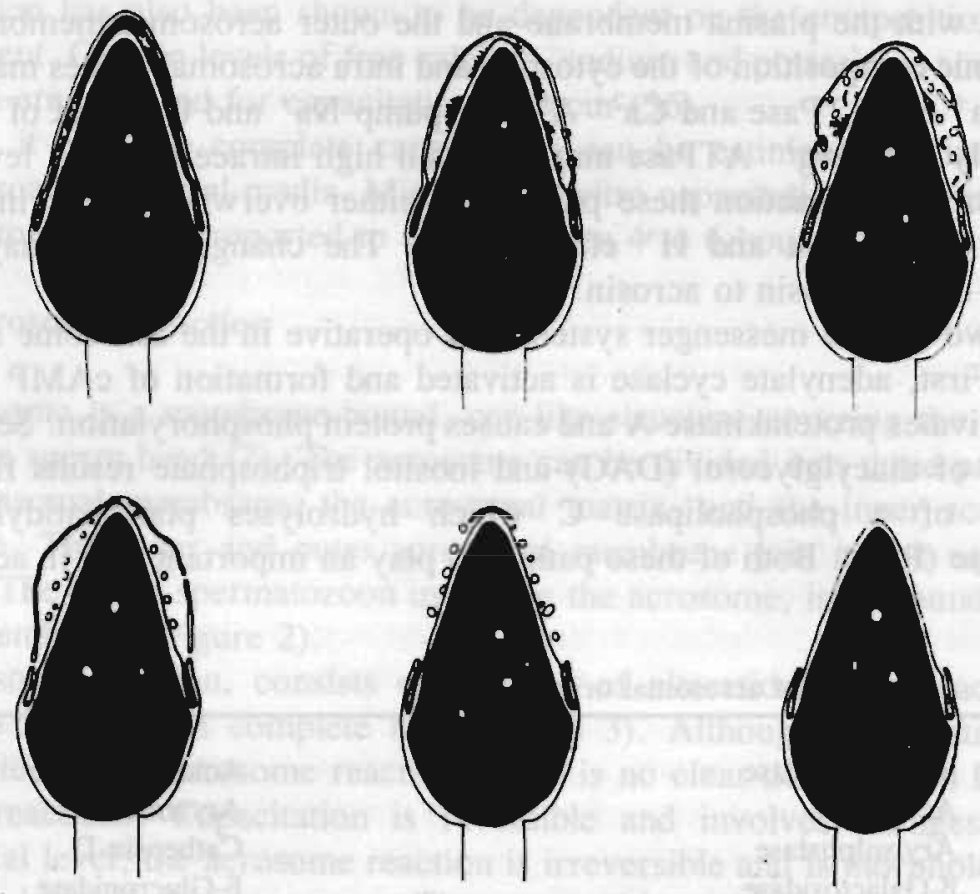

\subsection{Fertilization}

The fertilization begins with the penetration of the sperm through cumulus oophorus. Studies have shown that only capacitated spermatozoa that retained their acrosomal caps are able to enter the cumulus (103). Hyaluronidase plays an important role on cumulus penetration. Talbot, and, Zao and coworkers 
$(92,108)$ ventured the opinion that the surface of hamster spermatozoa carries hyaluronidase, which, in part, originates from the male genital tract. This surface hyaluronidase, but not the acrosomal hyaluronidase, aids in sperm passage through the cumulus. However, the possibility that acrosomal enzymes escape through the outer and plasma membranes prior to their penetration and that these enzymes other than hyaluronidase take part in cumulus lysins should not be ignored $(32,71,90)$.

After passing the cumulus spermatozoa attach firmly to the zona pellucida prior to its penetration. This strong binding was first demonstrated by Gwatkin and Williams (40). It consists of the interaction between zona and sperm plasma membrane receptors. The zona glycoprotein ZP3, is the primary zona receptor which binds spermatozoa prior to the acrosome reaction, ZP2 is the secondary sperm receptor which binds to the spermatozoa after the acrosome reaction (94). The acrosome reacted spermatozoon, in the act of passing through the zona, loses its acrosomal components except for the equatorial segment and the inner acrosomal membrane. Therefore, these two components allow the acrosomal enzymes to be in direct contact with the zona pellucida. However, sperm tail movement is an essential additional mechanical factor and important of passing through the zona. Two hypotheses have been put forward (103). The mechanical hypothesis explains sperm penetration as a predominantly mechanical activity. The only function of the acrosomal enzymes are to expose the inner acrosomal membrane. Movement of the sperm tail forces the spermatozoon through the zona mechanically. The enzymatic hypothesis states every step of the passage to be enzyme dependent. Surface and acrosomal hyaluronidase are responsible for passing the cumulus. Acrosomal hyaluronidase digests the hyaluronic acid in the outer region of the zona. Acrosin does not dissolve the zona per se, but hydrolyses specific zona glycoproteins to soften the zona. The enzymes on the inner acrosomal membrane cleave zona molecules and the spermatozoon is pushed through the zona by mechanical force. Most probably, spermatozoa use both mechanical and enzymatical means to pass through the zona (For review see 103)(Figure 4).

After passing the zona pellucida, the sperm head crosses the perivitelline space and becomes attached to the vitellus and is gradually incorporated into the vitellus (103). The first site of the sperm to fuse with the oocyte plasma membrane is the sperm plasma membrane above the equatorial segment (11). Next the sperm plasma membrane becomes part of the oocyte plasma membrane (Figure 4). Upon fusion with the spermatozoon, the oocyte becomes activated metabolically. The most easily recognizable signs of this activation are the exocytosis of the cortical granules and the resumption of the meiosis. Following the cortical granule activation, the oocytes undergoes the so called "zona reaction" which changes the penetrability characteristics of the zona proteins and prevent entry of additional spermatozoa (100). 
Figure 4. Schematic drawing of human metaphase I oocyte, and fertilization.

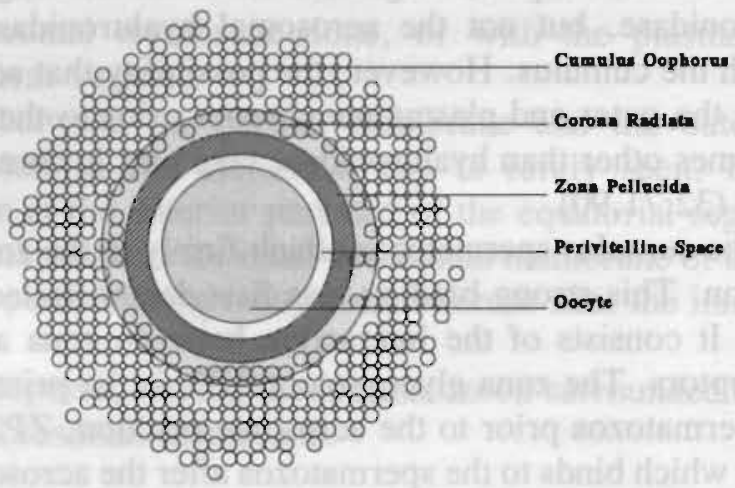

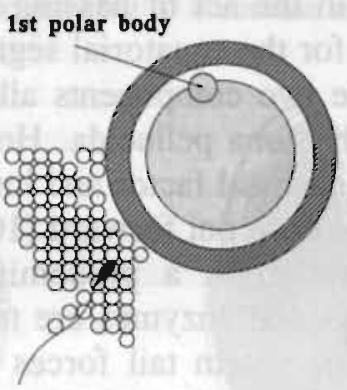

Sperm penetration through cumulus oophorus

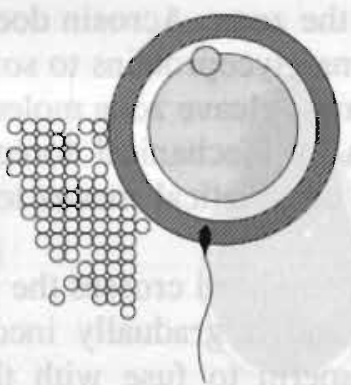

Sperm zona penetration

1st and 2nd polar body

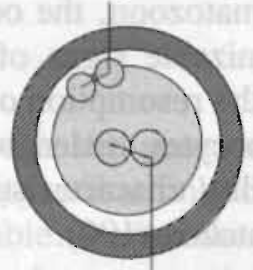

Male and female pronucleus

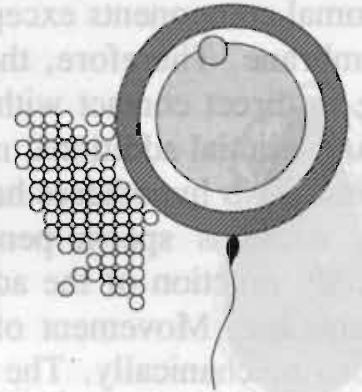

Sperm zona binding

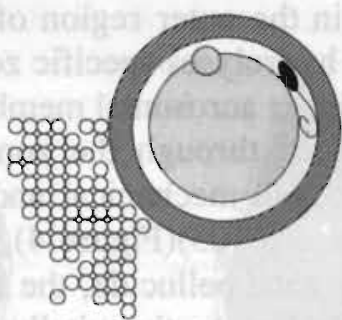

Sperm fusion with the oolemma 
Before fertilization the oocyte nucleus, was arrested at the metaphase of the second meiosis. After finishing this reduction division, the oocyte contains a haploid nucleus which transforms into the maternal pronucleus. Meanwhile, the sperm nucleus decondenses and transforms into the paternal pronucleus. The fully developed paternal and maternal pronuclei come into close contact and their nuclear envelopes disintegrate. Their chromosomes mingle. This mingling can be considered as the end of fertilization and the beginning of embryonic development (103).

\section{Diagnosis of Sperm Fertilizing Ability}

The high prevalence of male factor (48\%) (97) among infertile couples necessitates a complete andrological evaluation of the male partner of the couples who are consulting for infertility. In this section the diagnostic methods for sperm function will be discussed.

In addition to the conventional semen analysis, numerous tests have been developed in the last decade to evaluate the fertilizing potential of human sperm.

\subsection{Basic Semen Analysis}

Routine evaluation of male fertility starts with the basic semen analysis. The outlines of this investigation have been well defined by the WHO (96).

The macroscopic examination of the ejaculate includes the appearance, volume, liquefaction time, viscosity (consistency) and the $\mathrm{pH}$. However, isolated disturbances of above mentioned characteristics are rare (66).

The microscopic examination of the ejaculate is the cornerstone of the laboratory assessment. It should include the evaluation of density, motility, and morphology.

Sperm Density: Sperm density may be determined either after dilution by using a hemocytometer, or without dilution by using a Makler counting chamber. The latter has the advantage of being easy to handle and allowing more accurate counting since the depth of the chamber enables free horizontal movement of spermatozoa in one focal plane (61).

Motility: The WHO has defined four categories of motion characteristics of the spermatozoa: a- Rapid and linear progressive motility, b- Slow or sluggish linear or non-linear motility, c- non-progressive motility and d- immotility (96). Further information on motility can be found in Chapter IV.

Morphology: Sperm morphology has been taken into consideration as an effective index of male fertility since 1951 (60). However, the importance of 
morphology has gained momentum after the description of morphologically normal spermatozoa using strict criteria by Kruger and coworkers (52). The definition of strict criteria can be found in Chapter II. The role and the predictive value of morphology is the essential theme of this thesis and therefore will be discussed in detail from chapters II to V. In Appendix 2 the morphology evaluation using strict criteria (MEUSC) is summarized.

CASA: The need for a standard approach for semen analysis brought the computer assisted semen analysis (CASA) in use.

Many infertility centers use CASA for objective measurements of especially sperm density and motion parameters. Furthermore, additional motion characteristics such as velocity, linearity and lateral head displacement can not be substantiated with the conventional microscopic techniques. The WHO also included CASA into its latest manual for the examination of human semen (96). However, the systems suffer from practical and/or device deficiencies, making their operation often tedious and time consuming (25). More recently a computer program has been developed by Kruger and coworkers to evaluate the morphology of the human spermatozoa by CASA as well (54).

\subsection{Biochemical Tests}

Creatin Kinase: Creatin kinase is a key enzyme in the synthesis and transport of energy for spermatozoa. Huszar and coworkers have recently defined creatin kinase (CK) as a cellular marker of sperm quality (46). Higher sperm CK concentrations are related to a defect in sperm cytoplasmic extrusions, a key feature of spermatogenesis in the Sertoli cells. Furthermore, when oligospermic specimens were examined, those samples that subsequently proved to be fertile at IVF could be identified on the basis of their CK activity. Therefore, CK measurement was suggested to be used for differential diagnosis of the oligospermic samples (46).

Acrosomal enzyme activity: Since, acrosin and hyaluronidase are important enzymes for acrosome reaction and fertilization, it is not surprising that several techniques have been developed to evaluate the activity of these proteins.

The use of gelatinated slides to determine the proteolytic potential of spermatozoa has been described both for acrosin $(81,95)$ and for hyaluronidase activity (44). With this technique the halo formation around the sperm head indicates activity. Both the measurement of the diameters of the halos and the percentage of halo formation have been evaluated. However, the relationship between these tests and the IVF results has not been studied. Therefore, it is hard to speculate on the usefulness of gelatine slide tests in respect to fertilization in vitro.

The activity of acrosomal enzymes can also be measured by spectrophotometric determination, which was described by Goodpasture coworkers (39). Schill has 
recently reviewed the literature on this technique (82) and concluded that the predictability with respect to an individual patient is still limited because of the wide overlap in the range of the acrosin levels observed by all groups. Therefore, this technique should be considered in selected cases of teratozoospermia, particularly to confirm the diagnosis of globozoospermia (a complete absence of acrosomal structures due to a genetic defect that can be observed in about $0.1 \%$ of all andrological patients).

An alternative approach to evaluate the activity of enzymes involved in the acrosome reaction, is the assessment of the percentage of acrosome reacted spermatozoa in the sample. Although electron microscopy is the most effective method in this respect, it is impractical for routine analysis (58). Therefore, other techniques such as triple stain (91), monoclonal antibodies to acrosomal contents (101) chlortetracycline fluorescence and fluorescein isothiocyanate (FITC) labeled lectins such as Pisum sativum agglutinin (27) and peanut agglutinin $(56,70)$ have been developed. Among these, pisum sativum agglutinin has found a widespread use mainly because of the ease of performance.

With the help of polyclonal antibodies not only acrosin but also other sperm proteins can be evaluated. In a recent study from Senn and coworkers (83) acrosin, hyaluronidase, actin, dynein and tubulin have been evaluated using polyclonal antibodies. IVF couples and fertile donors were studied and the results were carefully evaluated by using ROC curves. The percentage of spermatozoa which reacted with the antibodies against acrosin and tubulin were the best indicators in respect to the IVF outcome. Therefore, this technique is suggested to be used as an additional and reliable tool in the IVF clinics.

\subsection{Immunological Tests}

At the onset of spermatogenesis, antigens appear on the sperm surface. However, the structural integrity of the epithelial linings of both male and female reproductive tracts provide a barrier to the penetration of sperm antigens into the systemic blood and lymphatic circulations (4). In men, injury or infection of the testis or epididymis and vasectomy may break down the barrier and evoke an anti sperm antibody (ASA) response.

Anti Sperm Antibodies: The most commonly used techniques for ASA detection in the fertility laboratories are mixed antiglobulin reaction (MAR test) and immunobead reaction (IBT test). For both of the techniques, $10 \%$ of tested spermatozoa with adherent particles is considered the normal lower limit by the WHO (96). The superiority of the MAR test lies in its practicality, and the requirement for a smaller volume (10 microliters for MAR against 0.5 to $2 \mathrm{ml}$ for IBT)(26). ASA detection is an important tool especially when there is no detectable cause for infertility. 
Sperm Cervical Mucus Interaction: The assessment can be made both in vivo (Post-coital Test (PCT)) and in vitro (Slide test, Kurzrok-Miller test, Kremer test).

Post-coital test was first described by Sims more than 100 years ago (47). Nevertheless, the debate over both the practicality and the sensitivity of the test remains (3). For reasons of convenience, in interpreting PCT's, and the need for standardization of sperm cervical mucus interaction testing many laboratories use an in vitro test. The Kremer test is the most commonly used (96). To prevent the probability of a false negative result, bovine cervical mucus, or human donor mucus and donor semen can be used as controls to further evaluate the sperm. Details of both in vivo and in vitro techniques may be found in the WHO laboratory manual (96).

Since cervical mucus serves to select sperm which have progressive motility (69) and normal morphology (78), it is important to evaluate these parameters initially.

\subsection{Functional Tests}

Hypoosmotic Swelling Test: The hypoosmotic swelling test (HOST) was introduced by Jeyendran and coworkers (48). It was designed to evaluate the functional integrity of the sperm membrane. The simplicity of the test allows the fertility clinics to include this test in their routine semen analysis protocol. Evaluation of the test and the correlation with in vitro fertilization outcome will be discussed further in the Chapter III of this thesis.

Sperm Penetration Assay: The zona-free hamster oocyte/sperm penetration assay (SPA) has often been used as a prognostic test to assess male fertility in vitro and also to quantify the improvement of sperm function after therapy (74). SPA was first introduced by Yanagimachi and coworkers (104) and measures the ability of the sperm to undergo capacitation, acrosome reaction, fusion with the oolemma and decondensation in the ooplasm (9). Although SPA methodologies varies from one laboratory to another, there is agreement in the literature that induction of capacitation and acrosome reaction prior to the test helps to decrease the false negative results and enhances the predictive value of the test $(1,2,55,106)$. The use of the hamster oocytes makes the test rather cumbersome and costly. In a study by Kruger and coworkers the correlation between SPA results and the conventional semen parameters in respect of the IVF outcome was studied. Their results show that morphology evaluation using strict criteria is an excellent predictor of SPA results and of human fertilization in vitro (53).

Hemizona Assay: The importance of spermatozoa binding to the zona pellucida and zona pellucida penetration has been previously discussed. Due to species specificity, human spermatozoa will bind firmly to no other zona pellucida 
except the human. Therefore, human spermatozoa binding can only be detected with the use of human oocytes. Burkman and coworkers have developed the hemizona assay (HZA) to test this binding (14). In the HZA, human oocytes are bisected by surgical manipulation, thus allowing for an internally controlled comparison of sperm binding (from patients versus fertile controls) to matching zona surfaces. Furthermore, zona penetration can also be evaluated with (HZA) (74).

The advantages of HZA are that: a- the two halves of hemizona are functionally and qualitatively equal, accordingly allowing a controlled comparison between the tested sperm and the donor; b- the limited number of limited available oocytes is amplified because an internally controlled test can be performed in a single oocyte; and c- because the oocytes are split microsurgically, even fresh oocytes can not lead to inadvertent fertilization and preembryo formation (35, 73,74).

\section{Statistical Analysis}

Introduction of new diagnostic tests also brought problems such as questioning the usefulness and the quality of the new tests. Both the usefulness and the quality of the diagnostic tests can be judged more reliably when there is a single accepted standard for the comparison of the test results (22). Metz has described the Receiver Operating Characteristic (ROC) curve, which can overcome many problems in medical decision making by describing the possible relationships between the probabilities of the various types of correct and incorrect decisions (67).

ROC curves display the implications of using different cut-off points to distinguish between diseased and non-diseased states. In the context of this thesis (Chapters II to V), we have used these terms to detect a fertile patient. Therefore, patients who were classified as true positive, in fact were those who had good test results (above cut-off point) and were able to fertilize their partners oocytes. True negative on the other hand were those with the poor test results (below cut-off point) and who failed to fertilize their partners oocytes. In the field of infertility, fertility and infertility are the commonly used definitions in place of non-diseased and diseased respectively. However, it should be kept in mind that since fertilization rates are not applicable to in vivo conditions, achieved pregnancies are used for the definition of fertile and infertile couples in in vivo conditions. For in vitro conditions, the occurrence of fertilization differentiates the fertile couple from the infertile couple. The necessity of using ROC curves for semen analysis has been emphasized both in this thesis (Chapters II to V) and in other studies $(24,43,76,22)$.

The reader can find a description of the methodology of ROC curves in Appendix 1. 


\section{Aims of This Study}

The evaluation of the role of human sperm morphology for the prediction of in vitro fertilization (IVF) outcomes was the starting point of this thesis. The aims of this study were;

1 To study the effect of normal morphology percentage, evaluated both with WHO criteria and with strict criteria, in IVF population (Chapter II).

2 A comparison of the conventional WHO criteria for morphology evaluation with the new strict criteria (MEUSC) for the IVF population (Chapters II to IV).

3 An evaluation of the predictive value of hypoosmotic swelling test (HOST) on the IVF outcomes and a comparison with the MEUSC (Chapter III).

4 An evaluation of the diagnostic value of semen analysis in IVF population (Chapter VI).

5 Morphology evaluation of the samples prepared for IVF and the prediction of IVF outcome (Chapter V).

6 An evaluation of sperm cyclic adenosinemonophosphate (cAMP) concentration both in pentoxifylline treated and in control groups (Chapter VI).

\section{References}

1. Aitken RJ, Wang YF, Liu J, Best F, Richardson DW (1983): The influence of medium composition, osmolarity and albumin content on the acrosome reaction and the fertilizing capacity of human spermatozoa: development of an improved zona-free hamster egg penetration test. Int J Androl, 6: 180-193.

2. Aitken RJ, Ross A, Hargreave T, Richardson DW (1984): Analysis of human sperm function following exposure to the ionophore A23187. Comparison of normospermic and oligospermic men. J Androl, 5: 321-329.

3. Alexander NJ (1990): Tests of spermatozoa function: Cervical mucus penetration. In: Human Spermatozoa in Assisted Reproduction. Acosta AA, Swanson RJ et al. (eds). Baltimore, Williams \& Wilkins; pg 106.

4. Alexander NJ, Ackerman SB, Windt ML (1990): Evaluation and preparation of spermatozoa for in vitro fertilization. In: Human Spermatozoa in Assisted Reproduction. Acosta AA, Swanson RJ et al. (eds). Baltimore, Williams \& Wilkins; pg 208.

5. Amann RP, Johnson L, Thompson DL (1976): Daily spermatozoal production, epididymal spermatozoal reserves and transit time of spermatozoa through the epididymis of the rhesus monkey. Biol Reprod, 15: 586.

6. Anderson RA, Feathergill KA, Zaneveld LJD (1991): Evidence for modulation of the human sperm acrosome reaction by G-proteins. J Androl, (Suppl): 43.

7. Austin CR, Bishop MWH (1958): Some features of the acrosome and perforatorium in mammalian spermatozoa. Proc R Soc Lond (Biol.), 149: 234.

8. Bailey ML, Grogan WM (1986): Protein kinase-mediated activation of temperaturelabile and temperature-stable cholesteryl ester hydrolases in the rat testis. J Biol 
Chem, 261: 7717-7722.

9. Barros C, Jedlicki A, Vigil P (1988): The gamete membrane fusion test to assay' the fertilizing ability of human spermatozoa. Hum Reprod, 3: 637-644.

10. Bedford JM (1970): Sperm capacitation and fertilization in mammals. Biol Reprod, 2(Suppl): 128.

11. Bedford JM, Cooper GW (1978): Membrane fusion events in fertilization of vertebrate eggs. In: Membrane Surface Reviews (Membrane Fusion). Vol 5. Poste $\mathrm{G}$ and Nicolson GL (eds). Amsterdam, North Holland; pg 65.

12. Brake A, Krause W (1992): Decreasing quality of semen. BMJ, 305: 1498.

13. Brooks DE (1981): Metabolic activity in the epididymis and its regulations by androgens. Physiol Rev, 61: 515-555.

14. Burkman LJ, Coddington CC, Franken DR, Kruger TF, Rosenwaks Z, Hogen GD (1988): The hemizona assay (HZA): development of a diagnostic test for the binding of human spermatozoa to the human hemizona pellucida to predict fertilization potential. Fertil Steril, 49: 688-697.

15. Burns RK (1961): Role of hormones in the differentiation of sex. In: Sex and Internal Secretions, ed 2, vol 1. Young WC (ed). Baltimore, Williams and Wilkins; pg 76.

16. Carlsen E, Giwercman A, Keiding N, Skakkebaek NE (1992): Evidence for decreasing quality of semen during past 50 years. BMJ, 305: 609-613.

17. Carlsen E, Giwercman A, Skakkebaek NE, Keiding N (1993): Decreasing quality of semen. BMJ, 306: 461.

18. Clermont Y, Leblond CP (1955): Spermiogenesis of man, monkey, ram, and other mammals as shown by the 'periodic acid Shiff' technique. Am J Anat, 96: 29.

19. Clermont $Y$ (1972): Kinetics of spermatogenesis in mammals: Seminiferous epithelium cycle and spermatogonial renewal. Physiol Rev, 52: 198.

20. Cocimano V (1991): Occupational factors and andrological problems. Arch Ital Urol Nefrol Androl, 63: 387-389.

21. Cohen J, Ooms MP, Vreeburg JT (1981): Reduction of the fertilizing capacity of epididymal spermatozoa by 5 alpha-steroid reductase inhibitors. Experentia, 37: 1031-1032.

22. Collins JA (1989): Male Infertility: The interpretation of the diagnostic assessment. In: The Year Book of Infertility. Mishell DR, Paulsen CA, Lobo RA (eds), Yearbook Medical Publishers inc.; pg 45.

23. Comhaire FH, Kretzer de DM, Farley TMM, Rowe PJ (1987): Towards more objectivity in diagnosis and management of male fertility; Results of a World Health Organization multicentre study. Int J Androl, 7(Suppl):1.

24. Comhaire FH, Vermeulen L, Hinting A, Schoonjans F (1988): Accuracy of sperm characteristics in predicting the in vitro fertilizing capacity of semen. J In Vitro Fertil Embryo Transf, 5: 326-331.

25. Comhaire FH, Huysse S, Hinting A, Vermulen L, Schoonjans F (1992): Objective semen analysis: has the target been reached? Hum Reprod, 7: 237-241.

26. Comhaire FH (1992): An approach to the management of male infertility. Bailliers Clin Endocrin Metab, 6: 435-450.

27. Cross NL, Morales P, Overstreet JW (1986): Two simple methods for detecting human sperm acrosome reactions. J Androl, 7: 28.

28. Davis BK, Byrne R, Bedigan K (1980): Studies on the mechanism of capacitation: albumin mediated changes in plasma membrane lipids during in vitro incubation of rat cells. Proc Nat Acad Sci, USA 77: 1546.

29. De Jonge CJ, Han HL, Mack SR, Zaneveld LJ (1991): Effect of phorbol diesthers, synthetic diacylglycerols and protein kinase $C$ inhibitor on the human sperm 
acrosome reaction. J Androl, 12: 62-70.

30. De Jonge CJ, Han HL, Mack SR, Zaneveld LJ (1991): Modulation of the human sperm acrosome reaction by effectors of the adenylate cyclase/cyclic AMP secondmessenger pathway. J Exp Zool, 258: 113-125.

31. Eicher EM, Washburn LL (1986): Genetic control of primary sex determination in mice. Ann Rev Genet, 20: 327-360.

32. Farooqui AA, Srivastava PN (1979): Isolation characterization and the role of rabbit testicular arylsulfatase. Biochem J. 181: 331.

33. Fawcett DW (1975): The mammalian spermatozoa. Dev Biol, 44: 394.

34. Feichtinger W (1991): Environmental factors and fertility. Hum Reprod, 6: 11701175.

35. Franken DR, Oehninger S, Burkman LJ, Coddington CC, Kruger TF, Rosenwaks Z, Acosta AA, Hodgen GD (1989): The hemizona assay (HZA): a predictor of human sperm fertilizing potential in in vitro fertilization (IVF) treatment. J In Vitro Fert Embryo Transf, 6: 44.

36. Fraser LR (1992): Requirements for successful mammalian sperm capacitation and fertilization. Arch Pathol Lab Med, 116: 345-350.

37. Ginsburg J, Hardiman P (1991): Ovulation induction with human menopausal gonadotrophins- a changing scene. Gyn Endocrinol, 5: 57-78.

38. Ginsburg J, Hardiman P (1992): Decreasing quality of semen. BMJ, 305: 1229.

39. Goodpasture JC, Polakoski KL, Zaneveld LJD (1980): Acrosin, proacrosin and acrosin inhibitor of human spermatozoa: extraction, quantitation and stability. $\mathrm{J}$ Androl, 1: 16.

40. Gwatkin RBL, Williams DT (1977): Receptor activity of the hamster and mouse solubilized zona pellucidae before and after the zona reaction. J Reprod Fertil, 49: 55.

41. Haas JGG, Cines DB, Schrieber AD (1980): Immunological infertility: Identification of patents with antisperm antibody. N Eng J Med, 303: 724.

42. Hertzberg VS, Lemasters GK, Hansen K, Zenick HM (1991): Statistical issues in risk assessment of reproductive outcomes with chemical mixtures. Environ Health Perspect, 90: 171-175.

43. Hinting A, Comhaire F, Vermeulen L, Dhont M, Vermeulen A, Vandekerckhove D (1990): Value of sperm characteristics and the result of in-vitro fertilization for predicting the outcome of assisted reproduction. Int J Androl, 13: 59-66.

44. Hirayama T, Hasegawa T, Hiroi M (1989): The measurement of hyaluronidase activity in human spermatozoa by substrate slide assay and its clinical application. Fertil Steril, 51: 330-334.

45. Howards SS, Johnson A, Jessee S (1975): Micropuncture and microanalytic studies of the rat testis and epididymis. Fertil Steril, 26: 13.

46. Huszar G, Vigue L, Corrales M (1990): Sperm creatine kinase activity in fertile and infertile oligospermic men. J Androl, 11: 40-46.

47. Jequier A, Crich J (1986): Semen analysis, a practical guide. Jequier A, Crich J (eds). England, Blacwell Scientific Publications; pg 122.

48. Jeyendran RS, Ven van der HH, Perez Pelaez M, Crabo BG, Zaneveld LJ (1984): Development of an assay to assess the functional integrity of the human sperm membrane and its relationship to other semen characteristics. J Reprod Fertil, 70: 219.

49. Johnson AL, Howards SS (1976): Intratubuler hydrostatic pressure in testis and in epididymis before and after long-term vasectomy in the guinea pig. Biol Reprod, 14: 371 . 
50. Jones WR (1980): Immunological infertility-Fact or fiction. Fertil Steril, 33: 577.

51. Josso N, Picard JY, Tran D (1979): The anti Müellerian hormone. In: : Morphogenesis and Malformation of the Genital System, The National Foundation, Birth Defects; Original Article Series, vol XIII. Balandau RJ, Bergsma D(eds). New York, Alan R Liss; p 59.

52. Kruger TF, Menkveld R, Stander FS, Lombard CJ, Van der Merwe JP, Van Zyl JA, Smith KA (1986): Sperm morphologic features as a prognostic factor in in vitro fertilization. Fertil Steril, 46: 1118-1123.

53. Kruger TF, Swanson RJ, Hamilton M, Simmons KF, Acosta AA, Matta JF, Oehniger S, Morshedi M (1988): Abnormal sperm morphology and other semen parameters related to the outcome of the hamster oocyte human sperm penetration assay. Int J Androl, 11: 107-113.

54. Kruger TF, DuToit TC, Franken DR, Oehninger S, Menkveld R, Lombard CJ, Acosta-AA (1993): A new computerized method of reading sperm morphology (strict criteria) is a s efficient as technician reading. Fertil Steril, 59: 202-209.

55. Lamb DJ, Johnson AR, Bassham BR, Lipschultz LI (1989): How the sperm penetration assay diagnoses fertility. Contemp Obstet Gyn, 33:108.

56. Lee MA, Trucco GS, Bechtol KB, Wummer N, Kopf GS, Blasco L (1987): Capacitation and acrosome reactions in human spermatozoa monitored by a chlortetcycline fluorescence assay. Fertil Steril, 48: 649-658.

57. Liu DY, Du Plessis YP, Nayudu PL, Johnston WI, Baker HW (1988): The use of in vitro fertilization to evaluate putative tests of human sperm function. Fertil Steril, $49: 272-277$.

58. Liu DY, Baker HW (1992): Tests of human sperm function and fertilization in vitro. Fertil Steril, 58: 465-483.

59. Mack SR, Everingham J, Zaneveld LJ (1986): Isolation and partial characterization of the plasma membrane from human spermatozoa. J Exp Zoo, 240: 127-136.

60. Macleod J, Gold RZ (1951): The male factor in fertility and infertility IV. Sperm morphology in fertile and infertile marriage. Fertil Steril, 2: 394.

61. Makler A (1978): A new chamber for rapid sperm count and motility estimation. Fertil Steril 30: 313.

62. Mancini RE (1976): Immunological aspects of testicular function. In: Monograms on Endocrinology, vol 9. New-York, Springer-Verlag; $p 1$.

63. McLaren A (1990): Sex determination. What makes a man a man? Nature, 346: 216217.

64. McNeal JE (1975): Developmental and comparative anatomy of the prostate. In: Benign Prostatic Hyperplasia. Grayhack JT, Wilson JD, Scherbenske MJ (eds). US Government Printing Office: p 1.

65. Means AR, Fakunding JL, Huckins C (1976): Follicle stimulating hormone, the Sertoli cell and spermatogenesis. Res Prog Horm Res, 32: 477.

66. Menkveld R, Kruger TF (1990): Basic Semen Analysis. In: Human Spermatozoa in Assisted Reproduction. Acosta AA, Swanson RJ et al. (eds). Baltimore, Williams \& Wilkins; pg 68.

67. Metz CE (1978): Basic principles of ROC analysis. Semin Nuc Med, 8: 283.

68. Mittwoch U (1992): Sex determination and sex reversal: genotype, phenotype, dogma and semantics. Hum Genet, 89: 467-479.

69. Mortimer D, Pandya IJ, Sawers RS (1986): Relationship between human sperm motility characteristics and sperm penetration into human cervical mucus in vitro. J Reprod Fertil, 78: 93-102.

70. Mortimer D, Curtis EF, Miller RG (1987): Specific labelling by peanut agglutinin 
of the outer acrosome membrane of the human spermatozoon. J Reprod Fertil, 81: 127-135.

71. Nagae T, Yanagimachi R, Srivastava PN, Yanagimachi H (1986): Acrosome reaction in human spermatozoa. Fertil Steril, 45: 701-707.

72. Narbaitz R, Tolnai G, Jolly E (1968): Ultrastructural studies on testicular biopsies from eighteen cases of hypospermatogenesis. Fertil Steril, 30: 679.

73. Oehninger S, Coddington CC, Scott R, Franken DR, Burkman LJ, Acosta AA, Hodgen GD (1989): Hemizona assay: assessment of sperm dysfunction and prediction of IVF outcome. Fertil Steril, 51: 665-670.

74. Oehninger S (1992): Diagnostic significance of sperm-zona pellucida interaction. Reprod Med Rew, 1: 57.

75. Page DC, Mosher R, Simpson EM, Fisher EMC, Mardon G, Polack J, McGillivray B, De-la-Chapelle A, Brown LG (1987): The sex-determining region of the human Y chromosome encodes a finger protein. Cell, 51: 1091-1104.

76. Peng HQ, Collins JA, Wilson EH, Wrixon W (1987): Receiver operating characteristics curves for semen analysis variables: methods for evaluating diagnostic tests of male gamete function. Gamete Res, 17: 229-236.

77. Perreault SD, Rogers BJ (1982): Capacitation pattern of human spermatozoa. Fertil Steril, 38: 258-260.

78. Pretorius E, Franken DR, De-Wet J, Grobler S (1984): Sperm selection capacity of cervical mucus. Arch Androl, 12: 5-7.

79. Russell LD, Clermont Y (1977): Degeneration of germ cells in normal, hypophysectomized and hormone-treated hypophysectomized rats. Anat Rec, 187: 347.

80. Sadler TW (1985): Gametogenesis. In: Langman's Medical Embryology, ed 5. Baltimore, Williams \& Wilkins; pg 20.

81. Schill WB, Feifel M, Fritz H, Hammerstein J (1981): Inhibitors of acrosomal proteinase as infertility agents a problem of acrosomal membrane permeability. Int $\mathbf{J}$ And, 4: 25.

82. Schill WB (1991): Some disturbances of acrosomal development function in human spermatozoa. Hum Reprod, 6: 969-978.

83. Senn A, Germond M, de Grandi P (1992): Immunofluorescence study of actin, acrosin, dynein, tubulin and hyaluronidase and their impact on in-vitro fertilization. Hum Reprod, 7: 841-849.

84. Silber SJ, Ord T, Balmaceda J, Patrizio P, Asch RH (1990): Congenital absence of the vas deferens. The fertilizing capacity of human epididymal sperm. N Engl J Med, 323: 1788-1792.

85. Sinclair AH, Berta P, Palmer MS, Hawkins JR, Griffiths BL, Smith MJ, Foster JW, Frischauf AM, Lovell Badge R, Goodfellow PN (1990): A gene from the human sex-determining region encodes a protein with homology to a cryopreserved DNAbinding motif. Nature, 346: 240-244.

86. Soderstrom RO, Andersson LC (1981): Identification of the autoantigen-expressing cells in rat testis. Exp Mol Pathol, 35: 332-337.

87. Steeno OP (1993): Occupational influences in male infertility. In: Abstract book of Andrology in Nineties. Ombelet W, Vandeput $\mathrm{H}$ et al. (eds), Genk.

88. Steptoe PC, Edwards RG, Purdy J (1980): Clinical aspects of pregnancies established with cleaving embryos grown in vitro. Br J Obs Gyn, 87: 757.

89. Suzuki F, Nagano T (1986): Microvasculature of the human testis and excurrent duct system: Resin-casting and scanning-electron microscopic studies. Cell Tissue Res, 243: 79-89.

90. Szollosi D. Huter RHF (1978): The nature and the occurrence of the acrosome 
reaction in spermatozoa of the domestic pig. Susscrofa J Annat 127: 33.

91. Talbot P, Chacon RS (1980): A triple stain technique for scoring acrosome reaction of human sperm. J Exp Zoo, 215: 201.

92. Talbot P (1985): Sperm penetration through oocyte investments in mammals. Am J Anat, 174: 331 .

93. Tummon I, Mortimer D (1992): Decreasing quality of semen. BMJ 305: 1228.

94. Wassarman PM (1988): Fertilization in mammals. Sci Am, 259: 78-84.

95. Welker B, Bernstein GS, Diedrich K, Nakamura RM, Krebs D (1988): Acrosomal proteinase activity of human spermatozoa and relation of results to semen quality. Hum Reprod, 3(Supp 2): 75.

96. WHO laboratory manual for the examination of human semen and sperm cervical mucus interactions (1992). Cambridge, Cambridge University Press, 3rd edn.

97. WHO manual for the standardized investigation and diagnosis of the infertile couple (1993). Rowe PJ, Hargreave TB, Mellows HJ, Comhaire FH (eds), Cambridge, Cambridge University Press.

98. Wilson JD (1978): Sexual Differentiation. Annu Rev Physiol, 40: 279.

99. Wilson JD, GeorgeFW, Griffin JE (1981): The hormonal control of sexual development. Science, 211: 1278.

100. Wolf DP (1981): The mammalian egg's block to polyspermy. In: Fertilization and embryonic development in vitro. L Mastroianni and B.J. Biggers (eds). New York, Plenum Press; pg 183.

101. Wolf DP, Bold J, Byrd W, Bechtol KB (1985): Acrosome status evaluation in human ejaculated sperm with monoclonal antibodies. Biol Reprod, 32: 1157-1162.

102. Working PK (1988): Male reproductive toxicology: comparison of the human to animal models. Environ Health Perspect, 77: 37-44.

103. Yanagimachi R (1988): Mammalian Fertilization. In: The Physiology of Reproduction. Knobil E and Neil J et al. (eds). New York, Raven Press; pg 135.

104. Yanagimachi R, Yanagimachi H, Rogers BJ (1976): The use of zona-free animal ova as a test system for the assessment of the fertilizing capacity of human spermatozoa. Biol Reprod, 15: 471.

105. Yates CA, De-Kretser DM (1987): Male-factor infertility and in vitro fertilization. J In Vitro Fert Embryo Transf, 4: 141-147.

106. Yee-B, Cummings-LM (1988): Modification of the sperm penetration assay using human follicular fluid to minimize false negative results. Fertil Steril 50: 123-128.

107. Zaneveld LJD, de Jonge CJ, Anderson RA, Mack SR (1991): Human sperm capacitation and acrosome reaction. Hum Reprod, 6: 1265-1274.

108. Zao PZR, Meizel S, Talbot P (1985): Release of hyaluronidase an beta-N-acetylhexoaminidase during in vitro incubation of hamster sperm. J Exp Zoo, 234: 63-74. 


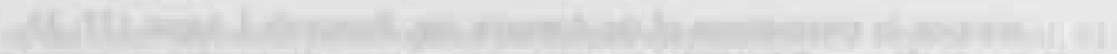

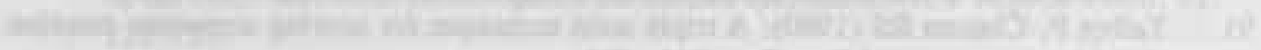

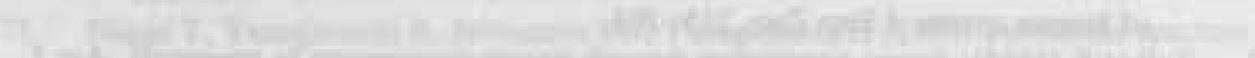
Iath

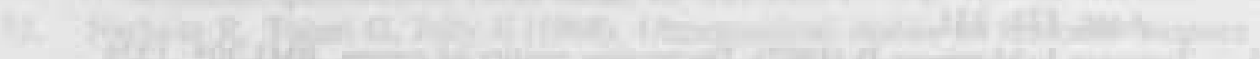

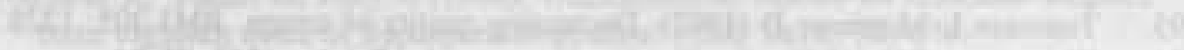

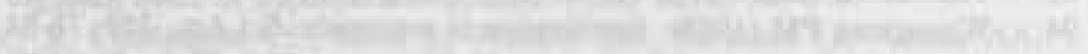

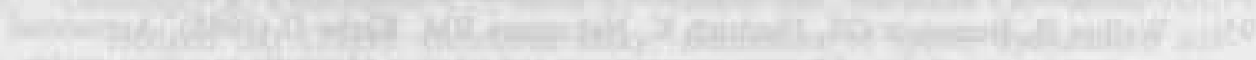

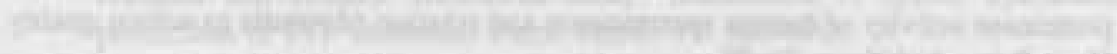

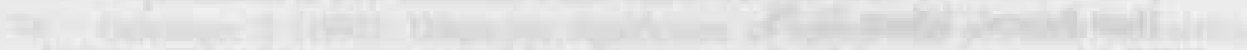

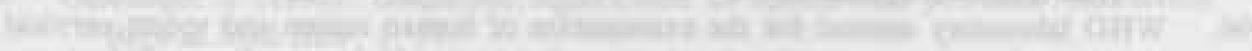

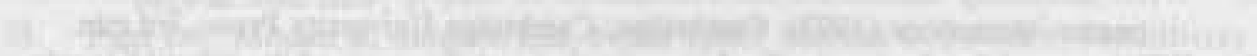

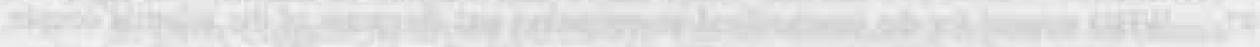

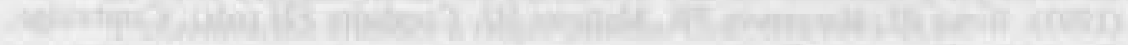

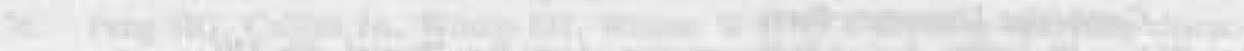

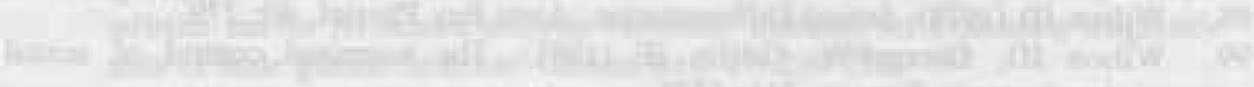

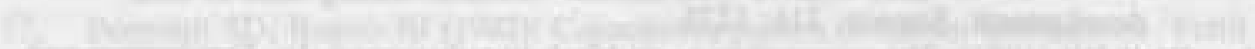

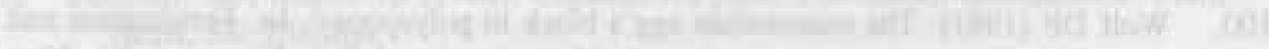

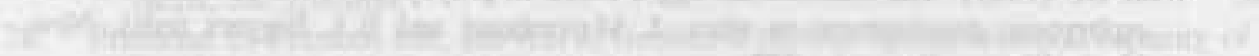

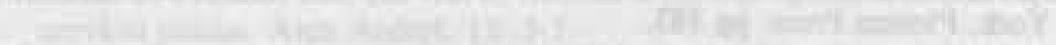

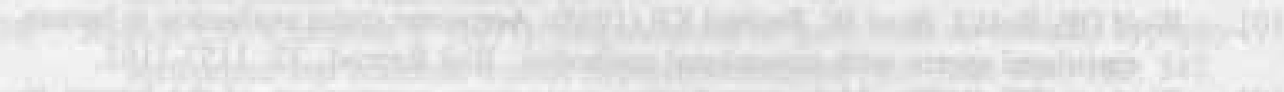

Wh the 1.

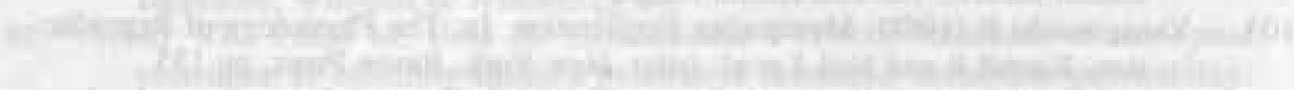

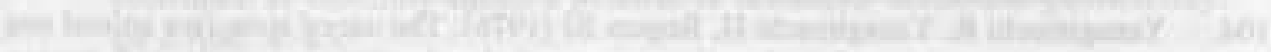

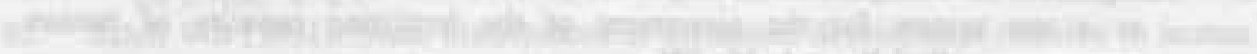
-

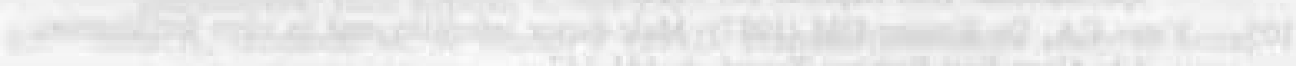

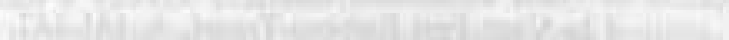




\title{
Evaluation of human sperm morphology using strict criteria after Diff-Quik staining: correlation of morphology with fertilization in vitro
}

\author{
M.E. Enginsu, J.C.M. Dumoulin, M.H.E.C. Pieters, M. Bras,
}

J.L.H. Evers, J.P.M. Geraedts. Human Reproduction 6, 854-858, 1991.

New, very strict criteria were used after Diff-Quik staining for evaluating sperm morphology. The results of morphology scoring were correlated with the fertilization rate in vitro. Semen samples from 64 men participating in an in vitro fertilization program were used for this study. All men had to have a sperm concentration of $\geq 20$ million/ml and a progressive motility of $>30 \%$. The morphology evaluation using strict criteria was performed on the same aliquot of semen as that used for in vitro insemination. If strict criteria showed that normal morphology was $\leq 4 \%$, the fertilization rate per oocyte was $23 \%$. If normal morphology was $\geq 11 \%, 77 \%$ fertilization occurred. For proportions of normal morphology between 4 and $11 \%$, the fertilization rate per oocyte was $59 \%(P<0.000001)$. Among all these morphology groups, classical semen parameters, such as the mean volume, the mean concentration and the mean motility, did not differ significantly except for the morphology evaluation using WHO criteria. The correlation with fertilization was better for morphology evaluation using strict criteria than for WHO. In conclusion, the method of evaluating sperm morphology based on very strict criteria allows for a more accurate prediction of the chance of fertilization in vitro. Further studies should be done to establish the most appropriate cut-off points for severely impaired, intermediate and high fertilization rates.

\section{Introduction}

A systematic study of human gamete interaction in vitro has become feasible since the introduction of in vitro fertilization (IVF) techniques. Results obtained by these techniques have brought into question the predictive value of conventional semen analysis (Davis et al., 1979; Albertsen et al., 1983; Rogers et al., 1983). However, sperm parameters have a significant influence on fertilization rates. Various sperm parameters and different sperm evaluation tests are advocated in the literature; laboratories are trying to develop their own standards to predict fertilization and pregnancy results.

The present study is based on the evaluation of sperm morphology using strict criteria after Diff-Quik staining. In spite of recent advances in clinical andrology, simple evaluation of the proportion of normal to abnormal sperma- 
tozoa in human semen is still subject to much debate in the diagnosis of male infertility (Kruger et al., 1986). One problem relates to the use of sperm morphology as a tool in evaluating the semen sample. A new method using strict criteria to evaluate sperm morphology has been reported to correlate well with fertilization rates in vitro (Kruger et al., 1987a,b; 1988a,b). Compared with other staining techniques for evaluation of sperm morphology, a recently prepared mix of reagents called Diff-Quik stain has been shown to give the best results in visualizing individual spermatozoa (Kruger et al., 1987b).

This investigation was undertaken to determine the predictive value of the combination of both, evaluation and staining methods with respect to prediction of fertilization in vitro in couples in whom the male partner had normal sperm density and progressive motility.

\section{Materials and Methods}

\section{Patients}

A total of 64 IVF couples was included in this study. The couples had one of the following indications: tubal pathology (30), unexplained infertility (23), endometriosis (6). Five more couples were included, diagnosed as male subfertility based on their previous semen analysis but having normal values on the day of oocyte collection. To allow for studying the contribution of morphology per se and to minimize interference with low sperm density and/or motility, all sperm samples used in this study had to have a sperm concentration of $\geq 20$ million $/ \mathrm{ml}$ and progressive motility of $30 \%$ in the semen analysis.

\section{In vitro fertilization}

Multiple follicular development was induced using a mild stimulation protocol with clomiphene citrate (CC) $50 \mathrm{mg}$ daily for 5 days starting at day 3 of the menstrual cycle and human menopausal gonadotropin (HMG), 150 units i.m. daily starting at day 6 . The growth of the follicles was followed ultrasonographically on day 3 , day 8 and daily from day 10 . As additional parameters, the serum estradiol $\left(E_{2}\right)$ and luteinizing hormone (LH) levels were measured daily from day 8 onwards. Human chorionic gonadotropin (HCG; 10.000 units) was given intramuscularly on the evening of the day the mean diameter of the dominant follicle reached $16 \mathrm{~mm}$, provided that no spontaneous $\mathrm{LH}$ surge was detected. Oocyte recovery was performed by vaginal ultrasound-guided aspiration 34 hours after HCG injection.

Whittingham's T6 medium (Quinn et al., 1984) supplemented with $10 \%$ inactivated maternal serum was used both for the culture of oocytes and embryos and for washing and incubation of the spermatozoa. 
Oocytes were cultured in an atmosphere of $5 \% \mathrm{CO}_{2}$ in air, which resulted in a $\mathrm{pH}$ of the culture medium of 7.4 after equilibration. The osmolality of this medium was adjusted to $285 \mathrm{mOsm} / \mathrm{kg}$. Semen was produced approximately two hours after the ovum retrieval. Immediately after liquefaction of the semen, liquefaction and viscosity were evaluated, the volume of the semen measured and the sperm concentration, percentage motility and agglutination evaluated, using Makler counting chamber. For each sample, a suspension of motile spermatozoa was prepared by a swim-up technique (Mahadevan and Baker, 1984) and incubated for another $2 \mathrm{~h}$. At $5 \mathrm{~h}$ after oocyte recovery, $\approx 50.000$ progressive motile spermatozoa were added to each oocyte. After incubation for 18-20 hours, the oocytes were checked for the presence of pronuclei as evidence of fertilization. The fertilized oocytes were transferred to fresh medium and cultured for another 24 hours. At $42-44$ hours after insemination, up to 4 embryos with the best morphological aspect were transferred to the uterus of the patient; any supernumerary oocytes were frozen.

\section{Semen Handling}

Conventional methods were used to evaluate the volume, sperm density, percentage of motility and morphology of the spermatozoa according to the guidelines of The World Health Organization (1987).

From each semen sample which was used for insemination, two semen smears were prepared. Prior to use, the slides used for semen smears were thoroughly cleaned with $100 \%$ methanol. In order to obtain as thin a smear as possible only $5 \mu \mathrm{l}$ of semen were used. The slides were air-dried for $\approx 3 \mathrm{~min}$, fixed for 15 $\mathrm{s}$ in Diff-Quik fixative $(1.8 \mathrm{mg} / \mathrm{L}$ triarylmethane in methyl alcohol) prior to staining with Diff-Quik solution 1 ( $1 \mathrm{~g} / \mathrm{L}$ xanthene in sodium azide-preserved buffer) for $10 \mathrm{~s}$ and with Diff-Quik solution $2(0.625 \mathrm{~g} / \mathrm{L}$ azure $\mathrm{A}$ and 0.625 $\mathrm{g} / \mathrm{L}$ methylene blue in buffer) for $5 \mathrm{~s}$. All Diff-Quik solutions were obtained from Dade Diagnostics, Miami, FL, USA. Between the fixing step and each of the staining steps, the excess solutions were drained from the slides by placing the slides vertically on absorbent paper. Morphology was evaluated, after staining the smears with Diff-Quik stain, as outlined in detail by Kruger et al. (1988a). Morphology evaluation according to the guidelines of the WHO were done in the same slides. At least 200 spermatozoa per patient were evaluated at a magnification of $x 1000$.

\section{Statistics}

The relationships between the percentages of spermatozoa with normal morphology and the fertilization rates were examined using a Chi-square test in a $2 \times 2$ table. The Chi-square test was used to test the null hypothesis that the relative frequencies of fertilizations in the different classes did not differ 
from the relative frequencies of fertilizations in the total group. The same test was also used to estimate whether significant differences existed between various cut-off points. The fertilization rate per oocyte below and above each individual cut-off point was calculated.

The relationships between the total volume of the semen sample, the density, and the percentage of motile spermatozoa were evaluated in different normal sperm morphology groups by using two-tailed Student's t-test.

The relationship between individual test results and fertilization rates was tested by Spearman's rank correlation coefficient. Receiver Operating Characteristic (ROC) curves were used for the assessment of the effectiveness of the morphology evaluation using strict criteria, as compared to morphology evaluation according to the WHO criteria, to predict fertilization.

Cohen's Kappa statistics were calculated to assess the agreement between each diagnostic test and the eventual fertilization, after correction for agreement by chance. Likelihood ratios were calculated to express the likelihood of fertilization over the likelihood of non-fertilization, given normal test results $(\mathrm{LR}+)$ or abnormal test results (LR-).

\section{Results}

The results of the 64 semen samples which were used for insemination in vitro were divided into two groups on the basis of their percentage normal sperm morphology, as assessed by the strict criteria of morphology and WHO criteria respectively. The fertilization rates were evaluated for each individual cut-off point. The ROC curve of the evaluation using strict criteria enclosed (below and to the right) a larger area than the WHO ROC curve, and therefore the most accurate. This was confirmed by calculating Cohen's Kappa, which was 0.439 for the best cut-off point of the strict criteria ROC curve (viz. 4\%), as compared to 0.365 for the best cut-off point of the WHO ROC curve (viz. $16 \%)$. After evaluating every possible cut-off point by Chi square analysis, the fertilization rates below and above $11 \%$ normal morphology according to strict criteria showed the highest significant difference $(\mathrm{P}<0.000001)$.

Severe impairment of fertilization rates was found in the group where the normal morphology according to strict criteria was $4 \%$ and below. The fertilization rate in the group with $4 \%$ normal morphology by strict criteria was $23 \%$ in 11 oocyte collections. Therefore, three groups were compared on the basis of their fertilization rates per oocyte, namely those of low $(0-4 \%)$, intermediate (5-10\%) and high (11-18\%) proportions of normal morphology. No abnormalities were noted with respect to the liquefaction, viscosity and agglutination of all the 64 semen samples. The total mean volume of the semen sample, mean sperm concentration and mean percentage of motile spermatozoa were not significantly different between the three groups of low, intermediate and high 
normal morphology according to strict criteria. However, morphology evaluation applying WHO criteria gave significant differences between these three groups $(\mathrm{P}<0.005)($ Table $\mathrm{I})$. The fertilization rate was $23 \%$ in the low group (11 oocyte collections) and 59\% in the intermediate group (15 oocyte collections), and this difference was significant $(\mathrm{P}<0.000001)$ (Table I). The fertilization rate in the intermediate group differed significantly from the $77 \%$ fertilization rate in the group with high percentage normal morphology (38 oocyte collections $)(\mathrm{P}<0.000001)($ Table I).

Table I. Comparison between different groups with low, intermediate and high percentages of normal morphology according to strict criteria.

\section{Low Intermediate High}

Normal morphology (\%)

using strict criteria

$0-4$

$3.3(0.6)$

$(n=11)$
$5-10$

$7.9(1.8)$

$(n=15)$
11-18

$14.2 \quad(1.7)$

$(n=38)$

Fertilization rate

(\%) (per oocyte)

Volume (ml)

$23^{a, b}$

$59^{a, c}$

$77^{\text {b.c }}$

Concentration (million/ml)

Motility

$4.1(2.0)$

$3.8(1.8)$

$4.3 \quad(2.2)$

$57.8(40.6)$

92.5(61.5)

$98.6(59.2)$

$47.3(13.8)$

$52.3(11.2)$

$55.8(13.4)$

Morphology (WHO)

$14.5(7.5)^{\text {d.e }}$

$26.5(9.5)^{e}$

$35.7(9.8)$ d,f

a,b,c, and d, P $<0.000001$; e and f, P $<0.005$

Table II. Comparison of two different techniques of sperm morphology evaluation on the basis of correlation with fertility in vitro (Spearman correlation coefficient, Cohen's kappa and likelihood ratios).

\section{Technique}

n

$\mathbf{r}$

P

kappa

LR+ LR -

Morphology evaluation

with WHO criteria

64

0.282

$<0.01$

0.365

2.2

0.17

Morphology evaluation

using strict criteria

$64 \quad 0.555$

$<0.000005$

0.439

4.4

0.15

$\mathrm{r}=$ Spearman Correlation Coefficient; kappa $=$ Cohen's kappa $(<0.400=$ poor agreement; $0.400-0.750=$ good agreement); $L R+=$ Likelihood of fertilization, given normal test result (2-5 = fair agreement); LR - = Likelihood of fertilization, given abnormal test result (0.10$0.20=$ good agreement)

The fertilization rates also differed significantly between the low and high 
groups $(\mathrm{P}<0.000001)$ as did morphology evaluation according to WHO criteria (Table I). The group with $>11 \%$ normal morphology according to strict criteria could not be divided into separate subgroups in relation to fertilization rates. Morphology evaluation according to strict criteria correlated better with the fertilization rate in vitro $(r=0.555$ and $P<0.000005)$ than did conventional

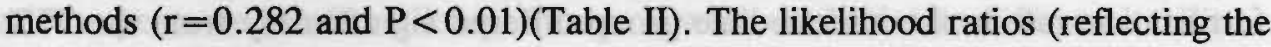
likelihood of fertilization over the likelihood of non-fertilization) for normal test results $(L R+)$ were 4.4 for the best cut-off point of strict criteria (4\%) and 2.2 for the best cut-off point of the WHO criteria (16\%). The related figures for abnormal test results (LR-) were 0.15 and 0.17 for the strict criteria and WHO respectively. The calculation of Cohen's Kappa was in favor of the strict criteria.

\section{Discussion}

A good correlation between normal morphology and fertilizing ability has been reported by many investigators (e.g. Rogers et al., 1983; Acosta et al., 1988; Chan et al., 1988; Kruger et al., 1988b; Scott et al., 1989; Dunphy et al., 1989; Turner et al., 1989; Hinting et al., 1990). Others were unable to find any such correlation (Aitken et al., 1982). Not all of the above-mentioned groups used the same evaluation or staining technique. Thus, the results of this study, especially the morphology evaluation using strict criteria, are not directly comparable with those obtained using other techniques.

The main difference between studies of morphology evaluation lies in the application of suitable criteria for evaluation. When morphology was evaluated according to the guidelines of WHO, there was a correlation between normal morphology percentages and fertilization in vitro $(r=0.282)$, though the correlation was worse than using strict criteria $(r=0.555)$. However, the mean percentages of spermatozoa with normal morphology are $<50 \%$ suggested by the WHO (1987)(Table I). Liu et al. (1988, 1989a,b) also found a good correlation between the percentages of normal morphology and fertilization in vitro. Their studies also give lower percentages than was suggested by the WHO. In two of their studies the patients were divided into two groups on the basis of their fertilization outcomes (Liu et al., 1988, 1989b). In the first study (Liu et al., 1988), the two groups were $\leq 50 \%$ and $>50 \%$ fertilization rate, and the mean number of normal morphology percentages using conventional methods were $32 \pm 2.9$ and $46 \pm 1.5$ respectively. In the second study the two groups were $0 \%$ and $>0 \%$ fertilization rate, with mean percentages with normal morphology of $20 \%$ and $36 \%$ respectively. These findings and ours indicate that the percentages of normal morphology which are suggested by WHO are rather high, at least for IVF patients.

Papanicolaou staining is widely used in evaluating the sperm morphology. 
Slides stained with Papanicolaou and Diff-Quik have almost the same quality, enabling the morphological status of spermatozoa to be evaluated in detail. However, the latter has advantages in having a clear background of the stained smears. Furthermore, Diff-Quik staining is a time saving procedure compared to other staining techniques. It makes use of quality controlled commercially prepared reagents and is easy to handle (Kruger et al., 1987b). A recently published study (Menkveld et al., 1990) elaborated on the importance of the strict criteria used for morphology evaluation.

Regardless of the staining technique used, the main correlation between the outcome of the fertilization in vitro and morphology of the spermatozoa, is due to the application of strict criteria for sperm morphology evaluation.

Normal sperm morphology, as evaluated by strict criteria, is a valuable tool for the prediction of the outcome of human IVF. Acosta et al. (1988) showed highly significant differences between the groups with $<14 \%$ and $>14 \%$ normal morphology with a fertilization rate of $49.5 \%$ and $88.3 \%$, respectively. They also showed that $<4 \%$ normal forms gave an extremely low fertilization rate of $7.6 \%$. Kruger et al. $(1986,1987 a, 1988 a, b)$ showed a good correlation between the fertilization rates in vitro and the percentages of normal morphological features in several studies. However, they also considered $>14 \%$ normal morphology as a cut-off point for good and $4 \%$ for poor fertilization patterns. These figures were confirmed later (Coetzee et al., 1989), for fertilization in vitro. In the present study, ROC curves showed that evaluation of sperm morphology according to strict criteria is more effective in predicting fertilization than evaluation according to WHO criteria. This was confirmed by the higher kappa statistic of 0.439 , indicating good agreement, compared to the WHO criteria kappa of 0.365 , which shows poor agreement between test result and fertilization. The higher LR + and the lower LR - of the strict criteria, as compared to the WHO criteria, qualify the morphology evaluation using strict criteria as the superior diagnostic test.

In our study, there was no further significant difference in fertilization rates among subgroups of normal morphology above $11 \%$. No matter how one uses the strict criteria or the published guidelines of evaluation techniques for different semen parameters, a standardization procedure for every new protocol still plays an important role in semen sample evaluation. This led us to establish our own cut-off point rather than taking a previously published value. All possible cut-off points were evaluated according to the accompanying fertilization rates. In our study, severe impairment was found with $23 \%$ fertilization rate in the group of patients who have a normal morphology of $4 \%$ or below. Therefore, in our hands, $4 \%$ is a suitable cut-off point for identifying IVF couples who are at risk of highly impaired fertilization rates.

It is our opinion that the difference between our cut-off points and those previously published are due to inter-laboratory differences. Since the use of strict criteria is very important for prediction of fertilization, the definition of 
normal spermatozoa should be standardized. We showed that evaluation according to strict criteria after Diff-Quik staining is the appropriate technique for morphology evaluation. Although it is hard to define normal spermatozoa, the most appropriate definition would be the one which was recently published by Menkveld et al. (1990), excluding all the borderline forms. Their results show that the evaluation of normal morphology requires even stricter criteria. This may be an additional explanation for our different cut-off points for low, intermediate and high fertilization rates.

From a clinician's point of view it is important to be able to decide which couples should be accepted for an IVF program, as it is a costly therapeutic procedure and both physically and psychologically stressful to patients. It is therefore important to classify the patients as subfertile and fertile. For the time being, there is no test available to draw a definite line between subfertile and infertile patients.

In conclusion, the method of morphology evaluation using strict criteria combined with Diff-Quik staining is very valuable for the prediction of the chance of fertilization in the individual couples. The differences between this study and others emphasize the necessity for every laboratory to establish its own standard cut-off points for predicting fertilization rates.

\section{Acknowledgements}

We thank Mrs. Anne Jansen for her secretarial assistance, and Mrs. Marij Bergers for technical assistance.

\section{References}

Acosta AA, Oehninger S, Morshedi M, Swanson RJ, Scott R, Irianni F (1988): Assisted reproduction in the diagnosis and treatment of the male factor. Obstet Gynecol Survey, 44: 1-18.

Aitken RJ, Best FSM, Richardson DW, Djahanbakhch O, Mortimer D, Tempelton AA, Lees MM (1982): An analysis of sperm function in cases of unexplained infertility: conventional criteria, movement characteristics, and fertilizing capacity. Fertil Steril, 38: 212221.

Albertsen PC, Chang TSK, Vindivich D, Robinson JC, Smyth JW (1983): A critical method of evaluating tests for male infertility. J Urol, 130: 467-475.

Chan SYW, Wang C, Chan STH, Ho PC, So WWK, Chan YF, Ma HK (1988): Predictive value of sperm morphology and movement characteristics in the outcome of in vitro fertilization of human oocytes. J IVF ET, 6: 142-148.

Coetzee K, Kruger TF, Menkveld R, Swanson RJ, Lombard CJ and Acosta,AA (1989): Usefulness of sperm penetration assay in fertility predictions. Arch Androl, 23: 207-212.

Davis G, Jouannet P, Martin-Boyce A, Spira A, Schwartz D (1979): Sperm counts in fertile and infertile men. Fertil Steril, 31: 453-455.

Dunphy BC. Neal ML, Cooke ED (1989): The clinical value of conventional semen analysis. Fertil Steril, 51: 324-329.

Hinting A, Comhaire F, Vermeulen L, Dhont M, Vermeulen A, Vandekerckhove D (1990): Value of sperm characteristics and the results of in-vitro fertilization for predicting the outcome of assisted reproduction. Int J Androl, 13: 59-66. 
Kruger TF, Menkveld R, Stander FSH, Lomberd CJ, Van der Merwe JP, Van Zyl JA, Smith K (1986): Sperm morphologic features as a prognostic factor in in vitro fertilization. Fertil Steril, 46: 1118-1123.

Kruger TF, Acosta AA, Simmons KF, Swanson RJ, Matta JF, Veeck LL, Morshedi M, Brugo S (1987a): New method of evaluating sperm morphology with predictive value for human in vitro fertilization. Urology, 30: 248-251.

Kruger TF, Ackerman SB, Simmons KF, Swanson RJ, Brugo SS, Acosta AA (1987b): A quick, reliable staining technique for human sperm morphology. Arch Androl, 18: 275277.

Kruger TF, Acosta AA, Simmons KF, Swanson JR, Matta JF, Oehniger S (1988a): Predictive value of abnormal sperm morphology in in vitro fertilization. Fertil Steril, 49: 112117.

Kruger TF, Swanson RJ, Hamilton M, Simmons KF, Acosta AA, Matta JF, Oehniger S, Morshedi M (1988b): Abnormal sperm morphology and other semen parameters related to the outcome of the hamster oocyte human sperm penetration assay. Int $\mathbf{J}$ Androl, 11: 107-113.

Liu DY, Du-Plessis YPD, Nayudu PL, Johnston WIH, Baker HWG (1988): The use of in vitro fertilization to evaluate putative tests of human sperm function. Fertil Steril, 49: 272-277.

Liu DY, Clarke GN, Lopata A, Johnston WIJ, Baker HWG (1989a): A sperm-zona pellucida binding test and in vitro fertilization. Fertil Steril, 52: 281-287.

Liu DY, Lopata A, Johnston WIH, Baker HWG (1989b): Human sperm-zona-pellucida binding, sperm characteristics and in vitro fertilization. Human Reprod, 4: 696-701.

Mahadevan M, Baker G (1984): Assessment and preparation of semen for in vitro fertilization; in Wood C, Trounson A (ed): Clinical In Vitro Fertilization. Berlin, Springer-Verlag, 1984, 83-97.

Menkveld R, Stander FSH, Kotze TJW, Kruger TF, Van Zyl JA (1990): The evaluation of morphological characteristics of human spermatozoa according to stricter criteria. Human Reprod, 5: 586-592.

Quinn P, Warnes GM, Kerin JF, Kirby C (1984): Culture factors in relation to the success of human in vitro fertilization and embryo transfer. Fertil Steril, 41: 202-209.

Rogers BJ, Bentwood BJ, Van Campen H, Helmbrecht G, Sodendahl D, Hale RW (1983): Sperm morphology assessment as an indicator of human fertilizing capacity. J Androl, 4: 119-125.

Scott RT Jr, Oehninger SC, Menkveld R, Veeck LL, Acosta AA (1989): Critical assessment of sperm morphology before and after double wash swim-up preparation for in vitro fertilization. Arch Androl, 23: 125-129.

Turner TW, Schrader SM, Peréz-Pelaez M, Karuhn RF, Van der Ven HH, Jeyendran RS (1989): Morphometric and volumetric comparisons of human spermatozoa. Arch Androl, 23: 201-206.

WHO, Laboratory Manual for the Examination of Human Semen and Sperm-Cervical Mucus Interactions (1987): Cambridge University Press, Cambridge 2nd edn, pp.9-11. 


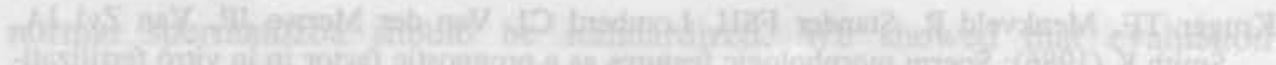

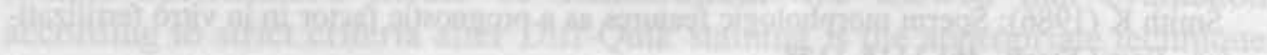

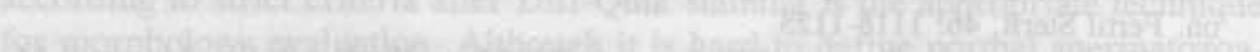

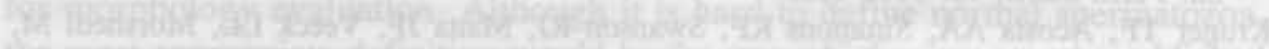

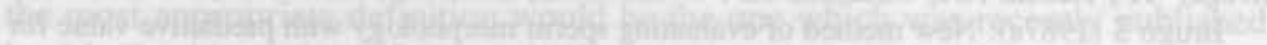

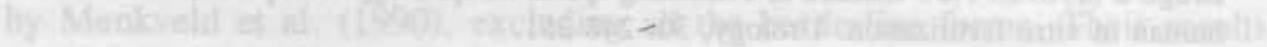

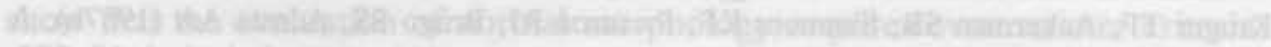

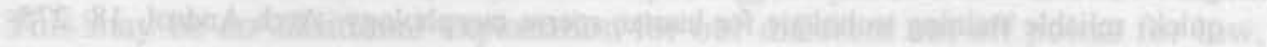

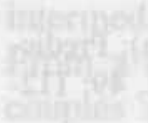

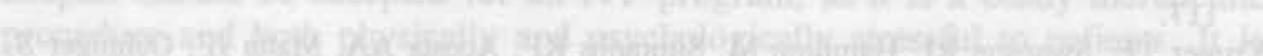

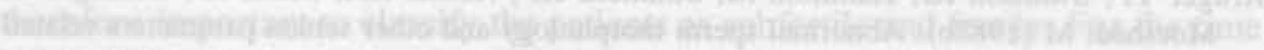

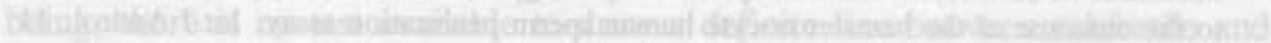
infortile mathats

(1) 11- no)

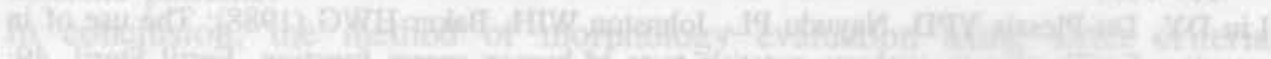

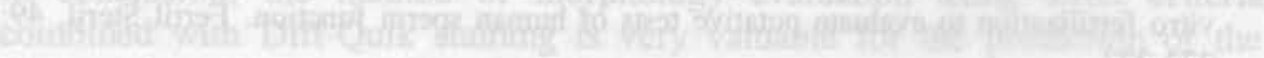

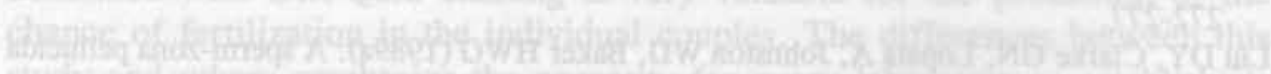

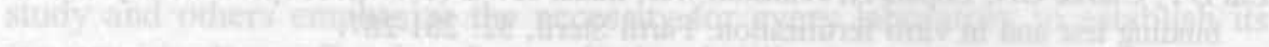

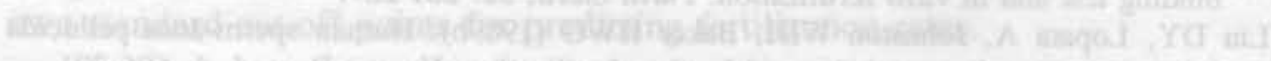

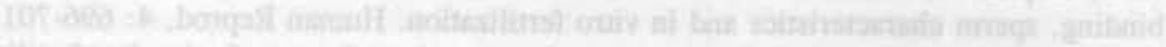

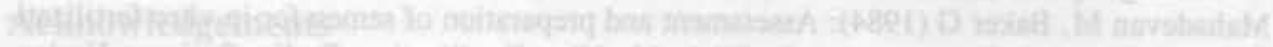

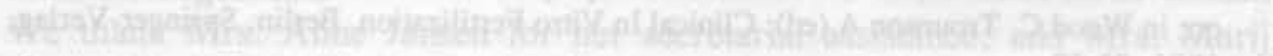

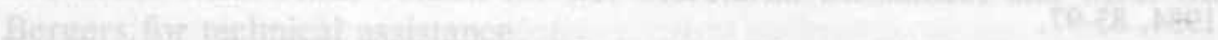

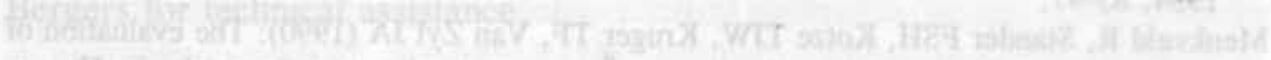

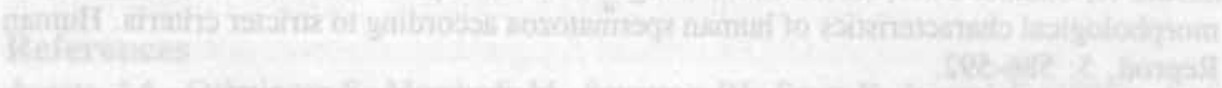

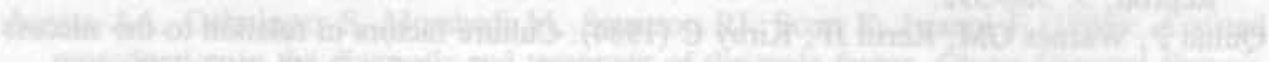

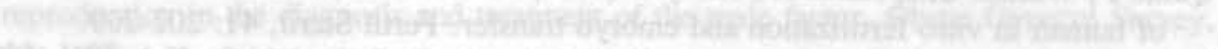

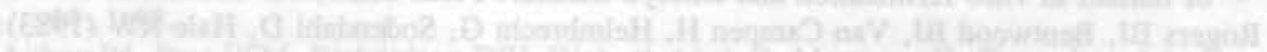

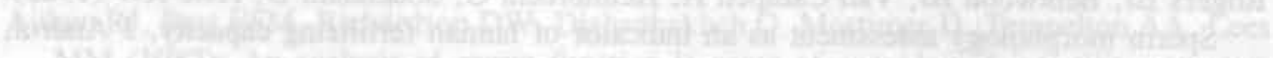

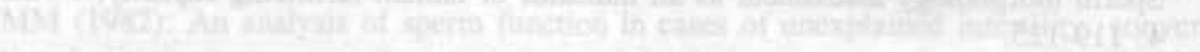

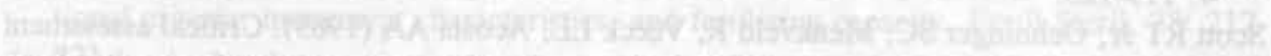

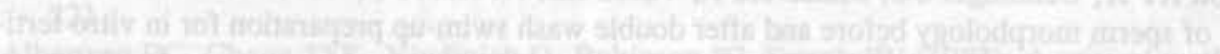

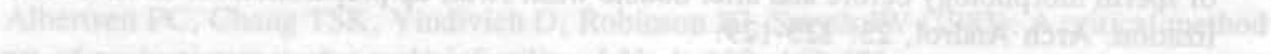

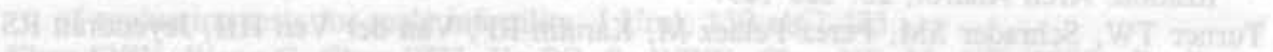

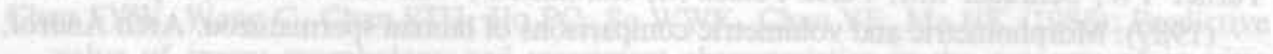

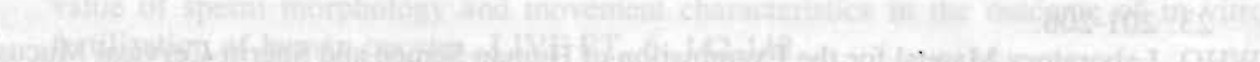

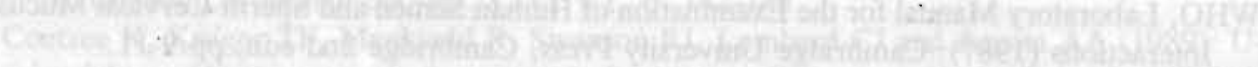

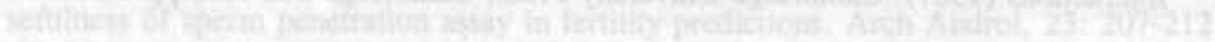

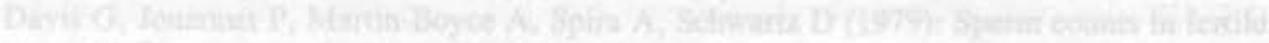

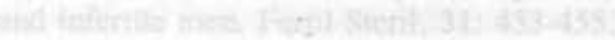

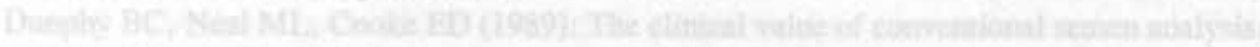

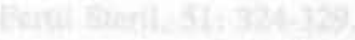

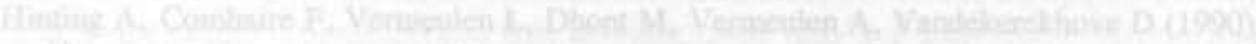

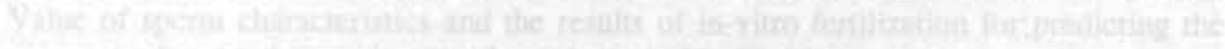

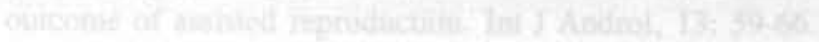




\title{
Comparison between the hypoosmotic swelling test and morphology evaluation using strict criteria in predicting in vitro fertilization (IVF)
}

\author{
M.E. Enginsu, J.C.M. Dumoulin, M.H.E.C. Pieters, M. Bergers, \\ J.L.H. Evers, J.P.M. Geraedts. Journal of Assisted Reproduction \\ and Genetics 9, 259-264, 1992.
}

Background: The role of male factor with respect to sperm morphology, progressive motility, and density is studied under in vitro conditions.

Methods: The semen samples of 67 males participating in an in vitro fertilization program were evaluated by the conventional WHO criteria of spermatogram, by morphology evaluation using strict criteria (MEUSC) and by the hypoosmotic swelling test (HOST). All sperm tests were performed in the original semen sample as delivered on the day of IVF, before further sperm treatment. The correlations between these parameters and the fertilization outcome were evaluated and their predictive values were calculated.

Results: When the patients were divided into two groups, namely fertile (fertilization rate per oocyte $>0 \%$ ) and infertile (fertilization rate per oocyte $=0 \%$ ), only mean sperm density and morphology were significantly different between the groups $(P<0.05)$. The correlation with fertilization rate in vitro was in favor of MEUSC.

Conclusions: Our results show that the HOST is inferior to MEUSC and conventional WHO sperm analysis in predicting fertilization in vitro.

\section{Introduction}

No reliable diagnostic test to predict the fertilization rate of human oocytes in vitro is available yet. Jeyendran et al. (1) developed the Hypo Osmotic Swelling Test (HOST), which was designed to evaluate the functional integrity of the sperm membrane. Because of its simplicity, the HOST has been particularly appealing and therefore has been studied in many IVF programs. Many groups claimed the HOST to be a good predictor of human IVF (2-9). Others have found only a weak correlation or no correlation between HOST results and IVF (10-15).

The percentage of morphologically normal spermatozoa is an important tool for IVF prediction: Liu et al.(16) and Chan et al.(17), using conventional WHO criteria for evaluation, showed a good correlation between sperm morphology and fertilization rate in vitro. Morphology evaluation using strict criteria was introduced by Kruger et al.(18). Subsequently the definition of normal 
spermatozoa was refined by Menkveld et al.(19), excluding all the borderline forms. In a recent study we compared morphology evaluation using strict criteria (MEUSC) with that of the using the conventional WHO criteria, and MEUSC appeared to have the best predictive value with respect to IVF (20). The present study was aimed at evaluating sperm swelling in response to hypoosmotic conditions and its correlation with human IVF. Moreover, HOST results were compared with the results of the semen analysis according to conventional WHO criteria and to MEUSC.

\section{Materials and Methods}

\section{Patients}

A total of 67 IVF couples was included in this study. The couples were admitted into the IVF program for one of the following indications: tubal pathology (33), unexplained infertility (21), endometriosis (7), male subfertility (3), immunological infertility (2), polycystic ovary syndrome (1).

\section{In vitro fertilization}

Multiple follicular development was induced using a mild stimulation protocol with clomiphene citrate (CC, Clomid: Gist-Brocades B.V., Leiderdorp, The Netherlands) $50 \mathrm{mg}$ daily for 5 days starting on day 3 of the menstrual cycle and human menopausal gonadotropin (hMG; Humegon; Organon, Oss, The Netherlands) 150 units im daily starting on day 6 . The growth of the follicles was followed ultrasonographically on day 3 and day 8 and daily from day 10 onward. As additional parameters, serum estradiol $\left(\mathrm{E}_{2}\right)$ and luteinizing hormone (LH) were measured daily from day 8 onward. Human chorionic gonadotropin (hCG, Pregnyl; Organon) 10.000 units was given im on the evening of the day on which the mean diameter of the dominant follicle reached $16 \mathrm{~mm}$, provided that no spontaneous LH surge was detected. Oocyte recovery was performed by vaginal ultrasound-guided aspiration $34 \mathrm{~h}$ after hCG injection.

Whittingam's T6 medium (21) supplemented with $10 \%$ inactivated maternal serum was used both for the culture of oocytes and embryos and for washing and incubation of the spermatozoa. Oocytes were cultured in an atmosphere of $5 \% \mathrm{CO}_{2}$ in air, which resulted in a $\mathrm{pH}$ of the culture medium of 7.4 after equilibration. The osmolality of this medium was adjusted to $285 \mathrm{mOsm} / \mathrm{kg}$. Semen was produced approximately $2 \mathrm{~h}$ after the ovum pick-up. An aliquot of this semen sample was used for further analysis by conventional methods, by MEUSC and by HOST. Of the remainder, a suspension of motile spermatozoa was prepared by a swim-up technique (22) and incubated for another $2 \mathrm{~h}$. At $5 \mathrm{~h}$ after oocyte recovery, approximately 50.000 progressive motile spermatozoa 
were added to each oocyte. After incubation for $18-20 \mathrm{~h}$, the oocytes were checked for the presence of pronuclei as proof of fertilization. The fertilized oocytes were transferred to fresh medium and cultured for another $24 \mathrm{~h}$. At 42 $44 \mathrm{~h}$ after insemination, up to 4 embryos with the best morphological aspect were transferred to the uterus of the patient; any supernumerary oocytes were froze (23).

\section{Semen Handling}

Conventional methods were used to evaluate the volume, sperm density, percentage of motility (slow, sluggish, linear, nonlinear movement or movement of the spermatozoa hampered by an obvious, clearly visible morphologic defect), progressivity (rapid and linear progressive motility), and morphology of the spermatozoa according to the guidelines of the World Health Organization (24). A Makler counting chamber was used for concentration and motility evaluation.

The HOST was performed by adding $0.1 \mathrm{ml}$ of whole semen which was produced on the day of ovum pick up and used for insemination, to $1 \mathrm{ml}$ of the hypoosmotic solution, which was prepared by dissolving $7.35 \mathrm{~g}$ of sodium citrate and $13.51 \mathrm{~g}$ fructose in $1 \mathrm{~L}$ distilled water. After the mixture was incubated for 30 minutes at $37^{\circ} \mathrm{C}$, the swelling of the sperm tail was evaluated according to the guidelines of Jeyendran et al. (1). The percentage of sperm that showed the typical tail configuration indicative of swelling was determined under the phase contrast microscope ( $40 \times 10$ magnification).

For the morphology evaluation using strict criteria two smears from each semen sample were prepared. Prior to use, the slides used for semen smears were thoroughly cleaned with $100 \%$ methanol. In order to obtain as thin a smear as possible only $5 \mu \mathrm{l}$ of semen was used. The slides were air-dried for approximately $3 \mathrm{~min}$, then fixed for $15 \mathrm{sec}$ in Diff-Quik fixative $(1.8 \mathrm{mg} / \mathrm{L}$ triarylmethane in methyl alcohol) prior to staining with Diff-Quik solution $1(1 \mathrm{~g} / \mathrm{L}$ xanthene in sodium azide-preserved buffer) for $10 \mathrm{sec}$ and with Diff-Quik solution $2(0.625 \mathrm{~g} / \mathrm{L}$ azure $\mathrm{A}$ and $0.625 \mathrm{~g} / \mathrm{L}$ methylene blue in buffer) for 5 sec. Diff-Quik solutions were obtained from Dade Diagnostics, Miami, USA. In between the fixing step and each of the staining steps, the excess solutions were drained from the slides by placing them vertically on bibulous paper. The morphology was evaluated as outlined in detail by Kruger et al. (25). At least 200 spermatozoa/per patient were evaluated at a magnification of $10 \times 100$.

\section{Statistics}

Patients were divided into two groups according to whether or not fertilization had occurred. The differences between the groups in the semen parametersvolume, sperm density, motility, morphology and hypoosmotic swelling of the 
spermatozoa-and the number of oocytes were studied using Student's t-test.

Correlations between individual test results and fertilization rates were evaluated by calculating Pearson's rank correlation coefficient.

Receiver Operating Characteristic (ROC) curves as described by Metz (26) were constructed for assessment of the effectiveness of each individual semen parameter in predicting fertilization.

Cohen's Kappa statistics (27) were calculated to assess the agreement between each diagnostic test and fertilization, after correction for agreement by chance for the best cut-off point.

Likelihood ratios (28) were calculated to express the likelihood of fertilization over the likelihood of non-fertilization, given normal LR + or abnormal LR - test results.

Figure 1. ROC curves for hypoosmotic swelling test and morphology evaluation using strict criteria

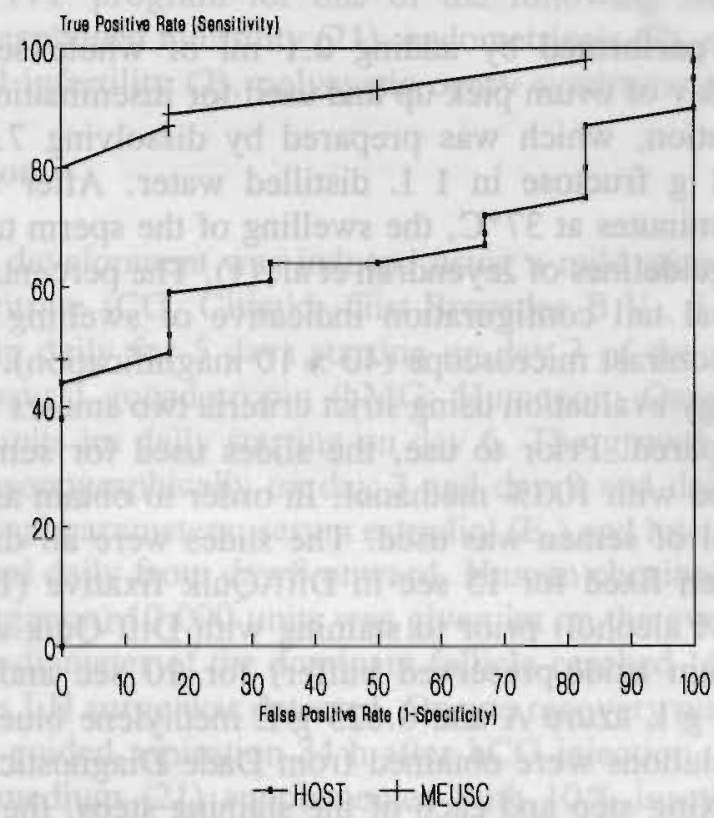

\section{Results}

No abnormalities were noted with respect to liquefaction, viscosity and agglutination of the 67 semen samples which were used for insemination in vitro.

The results of the 67 semen samples were divided into two groups on the basis of the fertilization outcome, i.e., fertile (fertilization rate $>0 \%$ per oocyte) and 
infertile (fertilization rate $=0 \%$ per oocyte). The mean numbers and standard deviations of the oocytes in the fertile group $(10.66 \pm 5.76)$ and in the infertile group $(8.67 \pm 6.02)$ were evaluated using Student's t-test to determine whether these two groups were comparable with one another. There was no significant difference between the two groups $(P=0.424$, Table I).

Table I. Comparison of the Numbers of Oocyte in Both Groups.

\begin{tabular}{lccc}
\hline & \multicolumn{3}{c}{ Fertilization Rate } \\
\cline { 2 - 3 } & $0 \%$ & $>0 \%$ & $P$ \\
\hline Mean number \pm SD & $8.67 \pm 6.02$ & $10.66 \pm 5.76$ & 0.424 \\
Range & $3-19$ & $2-24$ & \\
\hline
\end{tabular}

The means of volume, motility, progressive motility, and HOST did not show any significant difference, whereas sperm density, conventional morphology evaluation and MEUSC did ( $\mathrm{P}<0.05$; Table II).

The correlations between the various diagnostic tests for semen analysis and the fertilization rate in vitro were calculated. MEUSC appeared to have the highest correlation coefficient $(0.688)$; the HOST showed a much lower correlation $(0.334)$. Semen volume and sperm motility were not significantly correlated with IVF (Table II).

The best cut-off points for HOST $(60 \%)$ and the other parameters were calculated using ROC curves. The ROC curve of the MEUSC enclosed (below and to the right) a larger area than the ROC curves of any other semen parameters and, therefore, appeared to be the most accurate in predicting IVF (Figure I). This was confirmed by Cohen's kappa, which was 0.498 for the best cut-off point of the MEUSC ROC curve (4\%). HOST gave only poor agreement with a kappa value of 0.151 at the optimal cut-off point of $60 \%$ (Table III). The likelihood ratios (reflecting the likelihood of fertilization over the nonfertilization) for normal test results were 5.24 for results above the best cut-off point of MEUSC and 3.47 for values above the best cut-off point of the HOST respectively. The related figures for abnormal test results below the respective cut-off points were 0.13 and 0.49 for the MEUSC and HOST respectively, also in favor of MEUSC (Table III).

\section{Discussion}

With the introduction of human IVF, more direct comparisons can be made regarding the prediction of male infertility with laboratory tests. The percentage of normal human sperm morphology using both conventional and strict criteria for evaluation has been demonstrated to be a good predictor of IVF $(9,16$ - 
19,25 ). Comparing the two evaluation techniques, the superiority of the MEUSC is clear (20).

Table II. Comparison of the Semen Parameters (Mean \pm SD) of Both Groups.

\begin{tabular}{|c|c|c|c|}
\hline \multirow{2}{*}{ Parameter } & \multicolumn{2}{|c|}{ Fertilization Rate } & \multirow[b]{2}{*}{$\mathbf{P}$} \\
\hline & $\begin{array}{c}0 \% \\
(\mathrm{n}=6)\end{array}$ & $\begin{array}{c}>0 \% \\
(\mathrm{n}=61)\end{array}$ & \\
\hline Volu & $4.07 \pm 2.01$ & $4.50 \pm 2.18$ & NS \\
\hline sity (million/ml) & $33.33 \pm 26.39$ & $87.44 \pm 64.95$ & 0.05 \\
\hline Mo & $46.67 \pm 14.02$ & $54.34 \pm 15.26$ & NS \\
\hline Progressive motility (\%) & $22.17 \pm 7.28$ & $31.16 \pm 11.66$ & NS \\
\hline HOST $(\%)$ & $55.33 \pm 5.99$ & $60.33 \pm 13.08$ & NS \\
\hline Morphology (Con & $14.33 \pm 6.06$ & $30.51 \pm 11.36$ & 0.001 \\
\hline MEUSC (\%) & $3.67 \pm 1.37$ & $11.49 \pm 4.07$ & 0.0001 \\
\hline
\end{tabular}

Table III. Correlation of Semen Parameters With Fertilization Rate in Vitro ${ }^{a}$

\begin{tabular}{|c|c|c|c|c|c|c|}
\hline Parameter & $\mathbf{r}$ & $\mathbf{P}$ & Cut-off & kappa & $\mathbf{L R}+$ & LR - \\
\hline Volume (ml) & -0.04 & NS & 2.8 & 0.046 & 1.12 & 0.76 \\
\hline Sperm density (million/ml) & 0.365 & 0.01 & 13 & 0.448 & 0.90 & 0.11 \\
\hline Motility (\%) & 0.243 & NS & 40 & 0.118 & 1.48 & 0.52 \\
\hline Progressive motility (\%) & 0.368 & 0.01 & 40 & 0.268 & 1.74 & 0.26 \\
\hline HOST & 0.334 & 0.01 & 60 & 0.151 & 3.47 & 0.49 \\
\hline Morphology (conventional)(\% & 0.525 & 0.001 & 11 & 0.448 & 1.90 & 0.10 \\
\hline MEUSC & 0.688 & 0.001 & 4 & 0.498 & 5.24 & 0.13 \\
\hline
\end{tabular}

"r: Pearson's Correlation Coefficient; kappa: Cohen's kappa $(<0.400$, poor agreement: $0.400-0.750$, good agreement); LR $+:$ Likelihood of fertilization, given normal test result $(<2$, inoperative;2-5, fair agreement; 5-10, good agreement); LR-: Likelihood of fertilization, given abnormaltest result $(0.1-0.2$, good agreement; $0.2-0.5$, fair agreement; $>0.5$, inoperative).

Comparing likelihood ratios, the LR + of the HOST is better than that of the sperm density and morphology evaluation using conventional criteria. This indicates that there is a good agreement between the occurrence of IVF and the test results above the cut-off point. Our results show that $60 \%$ swelling is the most appropriate cut-off point for the HOST in pretreatment spermatozoa, which is in agreement with the other studies $(1,2)$. However, the kappa value demonstrated that most of the agreement was due to chance $(0.151)$. The correlation between sperm swelling in response to hypoosmotic conditions and IVF $(r=0.334)$ in this study, is much lower than that reported by Van der 
Ven et al.(2)( $r=0.56)$.

Chan et al. (17) demonstrated that, when evaluating standard semen parameters, only the percentage normal sperm morphology using conventional methods for evaluation was correlated with the fertilization rate of human oocytes and the HOST did not provide any additional predictive information.

Avery et al. (14) stated that the percentage of sperm showing the hypo-osmotic swelling response was not increased when the test was performed using 'swimup' preparations, but there was a significant increase in the mean motility, suggesting that there was no relationship between sperm motility and swelling response. They also demonstrated that the hypoosmotic sperm swelling test does not contribute to the prediction of the fertilizing capacity of human sperm in vitro.

Liu et al. (16) also could not find any relationship between the HOST results and the IVF of human oocytes, in both initial and prepared semen using the swim-up technique. They found good correlations between sperm morphology using WHO criteria, in both initial and inseminated semen, and IVF. There was also a good correlation between both groups, and the sperm morphology was significantly improved by the swim-up procedure.

Calderon et al. (29) studied human sperm morphology using strict criteria for evaluation technique on the prepared semen using Percoll gradients and concluded that this is a good method for preparing sperm for IVF samples by improving sperm motility and morphology.

Daya et al. (3) studied the HOST in the prepared semen by using the swim-up technique as a tool to predict the embryo cleavage. Also using the ROC curve analysis for evaluation, their results showed that the HOST results on sperm after swim-up strongly relate to the IVF. When the HOST results were corrected for the sperm density, the relationship was even closer. Their likelihood ratio of cleavage for normal test for the HOST alone was 1.8, which appears to be inoperative. A fair agreement (2.1) could be detected only when the HOST results were corrected for sperm density. Our results show a better agreement for HOST results in the initial semen sample but still they are in the fair agreement range (3.47). However, they are inferior to the MEUSC results, which is in the good agreement range (5.24). The percentage of sperm motility $(3,14,16,29)$, morphology $(16,29)$ and swelling response under hypoosmotic conditions increases when sperm preparation techniques (3), such as swim-up or Percoll gradients, are applied. This may lead one to use the actual insemination portion for prediction of IVF. Nevertheless, it is also important to be able to predict the IVF outcome on routine semen samples since this is the basic material for male fertility evaluation. Although there might be a better correlation between the quality of the actual insemination portion and IVF, our results show that, even without preparation techniques, the prediction is in the clinically acceptable range.

The number of subjects evaluated in this study, with six patients in the group 
with no fertilization, is indeed. However, this is due to the fact that the best comparison could only be made on the basis of absence of fertilization. The fact that the mean numbers of oocytes were not significantly different shows that actually these two groups were comparable with respect to the female contributions. Furthermore, the correlation coefficients back up the fact that, in all fertilization rates (from $0 \%$ to $100 \%$ ) regardless of the technique, percentage of normal morphology $(\mathrm{P}<0.001)$ is superior to that of HOST $(\mathrm{P}<0.01)$.

We therefore conclude that the HOST of individual native semen samples has no practical value in predicting eventual fertilization of human oocytes by prepared sperm cells in vitro.

\section{References}

1. Jeyendran RS, Van der Ven HH, Perez-Pelaez M, Crabo BG, Zaneveld LJ (1984): Development of an assay to assess the functional integrity of the human sperm membrane and its relationship to other semen characteristics. J Reprod Fertil, 70: 219-228.

2. Van der Ven HH, Jeyendran RS, Al-Hasani S, Perez-Pelaez M, Diedrich K, Zaneveld JD (1986): Correlation between human sperm swelling in hypoosmotic medium (Hypoosmotic swelling test) and in vitro fertilization. J Androl, 7: 190196.

3. Daya S, Gunby J, Kohut J (1989): Semen predictors of in vitro fertilization and embryo cleavage. Am J Obstet Gynecol, 161: 1284-1289.

4. Riedel HH, Hübner F, Ensslen SC, Bienek KW, Grillo M (1989): Minimal andrological requirements for in-vitro fertilization. Hum Reprod, 4 (Suppl): 73-77.

5. Shanis BS, Check JH, Bollendorf A (1989): Interpretation and misinterpretation of semen parameters. Arch Androl, 23: 213-227.

6. Check JH, Nowroozi K, Wu CH, Bollendorf A (1988): Correlation of semen analysis and hypoosmotic swelling test with subsequent pregnancies. Arch Androl, 20: 257-260.

7. Check JH, Epsyein R, Nowroozi K, Shanis BS, Wu CH, Bollendorf A (1989): The hypoosmotic swelling test as a useful adjunct to the semen analysis to predict fertility potential. Fertil Steril, 52: 159-161.

8. Mordel N, Mor-Yosef S, Margalioth E, Shemesh A, Samueloff ASA, Schenker JG (1989): The human sperm hypoosmotic swelling test: its practical application and suggestion for improvement. Int J Fertil. 5: 355-358.

9. Coetzee K, Kruger TF, Menkveld R, Lombard CJ, Swanson RJ (1989): Hypoosmotic swelling test in the prediction of male infertility. Arch Androl, 23: 131-138.

10. Barratt CLR, Osborn JC, Harrison PE, Monks N, Dunphy BC, Lenton EA, Cooke ID (1989): The hypo-osmotic swelling test and the sperm mucus penetration test in determining fertilization of the human oocyte. Hum Reprod, 4: 430-434.

11. Sjoblom P. Coccia E (1989): On the diagnostic value of the hypoosmotic swelling test in an in vitro fertilization (IVF) program. J IVF ET, 6: 41-43.

12. Chan SYW, Edgar JF, Chan MMC. Tsoi W, Wang C, Tang LCG, Tang GWK, Ho PC (1985): The relationship between the human sperm hypoosmotic swelling test, routine semen analysis, and the human sperm zona-free hamster ovum penetration assay. Fertil Steril, 44: 668-672.

13. Chan SYW, Wang C, Ng M. So WWK, Ho PC (1988): Multivariate discriminant 
analysis of the relationship between the hypo-osmotic swelling test and in-vitro fertilizing capacity of human sperm. Int J Androl, 11: 369-378.

14. Avery S, Bolton VN, Mason BA (1990): An evaluation of the hypoosmotic swelling test as a predictor of fertilizing capacity in vitro. Int $\mathrm{J}$ Androl, 13: 93-99.

15. Spittaler PJ, Tyler JPP (1985): Further evaluation of a simple test for determining the integrity of spermatozoal membranes. Clin Reprod Fertil, 30: 187-196.

16. Liu DY, Du-Plessis YPD, Nayudu PL, Johnston WIH, Baker HWG (1988): The use of in vitro fertilization to evaluate putative tests of human sperm function. Fertil Steril, 49: 272-277.

17. Chan SYW, Wang C, Chan STH, Ho PC (1990): Differential evaluation of human sperm hypoosmotic swelling test and its relationship with the outcome of in-vitro fertilization of human oocytes. Hum Reprod, 5: 84-88.

18. Kruger TF, Menkveld R, Stander FSH, Lomberd CJ, Van der Merwe JP, Van Zyl JA ,Smith K (1986): Sperm morphological features as a prognostic factor in in vitro fertilization. Fertil Steril, 46: 1118-1123.

19. Menkveld R, Stander FSH, Kotze TJW, Kruger TF, Van Zyl JA (1990): The evaluation of morphological characteristics of human spermatozoa according to stricter criteria. Hum Reprod, 5: 586-592.

20. Enginsu ME, Dumoulin JCM, Pieters MHEC, Bras M, Evers JLH, Geraedts JPM (1991): Evaluation of human sperm morphology using strict criteria after DiffQuik staining: Correlation of morphology with fertilization in vitro. Hum Reprod, 6: 854-858.

21. Quinn P, Warnes GM, Kerin JF, Kirby,C (1984): Culture factors in relation to the success of human in vitro fertilization and embryo transfer. Fertil Steril, 41: 202209.

22. Mahadevan M, Baker G (1984): Assessment and preparation of semen for in vitro fertilization; in Wood C, Trounson A (ed): Clinical In Vitro Fertilization. Berlin, Springer-Verlag, 83-97.

23. Dumoulin JCM, Evers JLH, Offermans JPM, Bras M, Pieters MHEC, Geraedts JPM (1990): Human in vitro fertilization using spermatozoa capacitated in hyperosmotic media. Gyn Obs Inv, 30: 165-168.

24. WHO, Laboratory Manual for the Examination of Human Semen and Sperm-Cervical Mucus Interactions(1987): Cambridge University Press, Cambridge 2nd edn, pp.911.

25. Kruger TF, Acosta AA, Simmons KF, Swanson JR, Matta JF, Oehniger S (1988): Predictive value of abnormal sperm morphology in in vitro fertilization. Fertil Steril, 49: 112-117.

26. Metz C (1978): Basic principles of ROC analysis. Semin Nucl Med, 8: 283-298.

27. Fleis JL (1981): Statistical Methods for Rates and Proportions. Toronto, John Wiley \& Sons , 56 .

28. Collins JA (1987): Diagnostic assessment of the infertile male partner. Curr Probl Obstet Gynecol Infertil, 10: 175-224.

29. Calderon G, Veiga A, Torello MJ, Vidal E, Martinez F, Barri PN (1991): Do Percoll gradients improve sperm morphology. Hum Reprod, 6(Suppl): 39. 


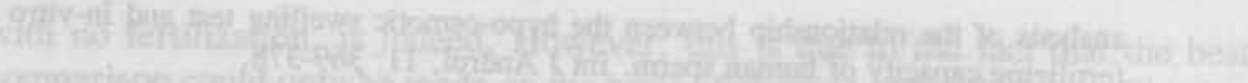

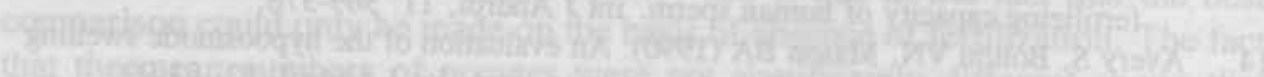

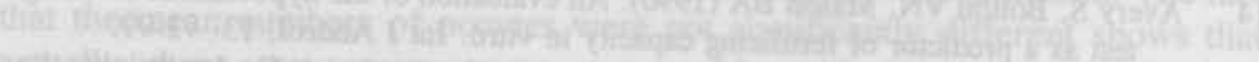

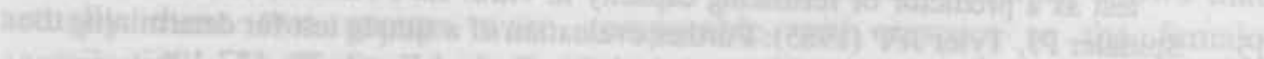

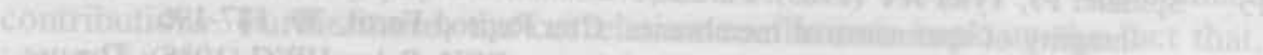

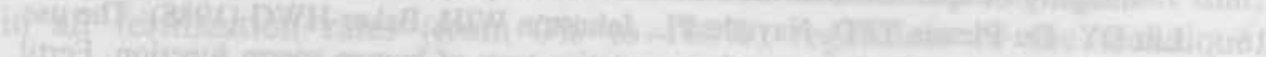

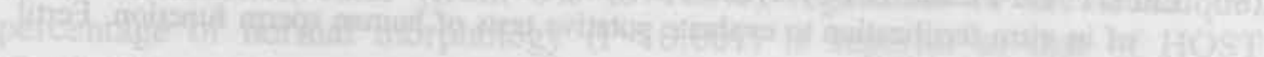

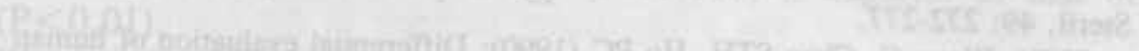

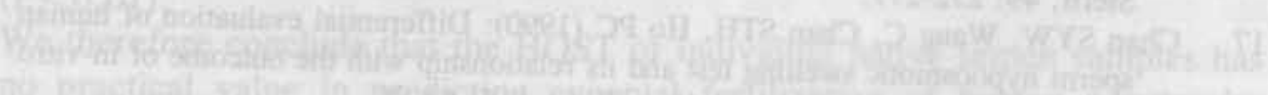

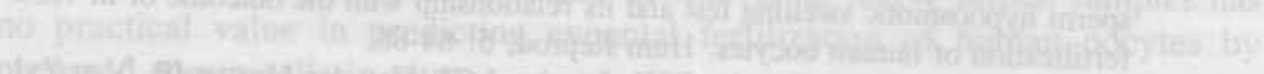

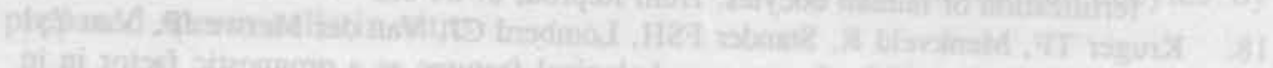

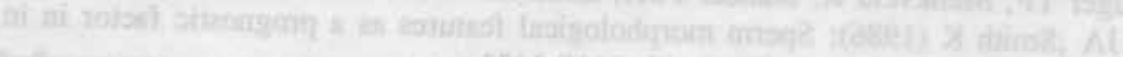

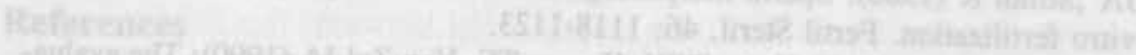

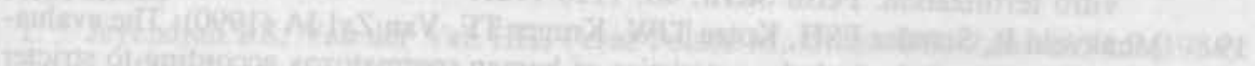

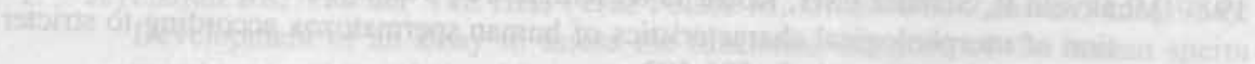

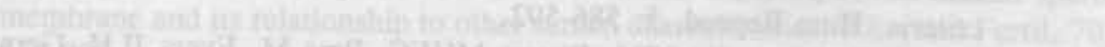

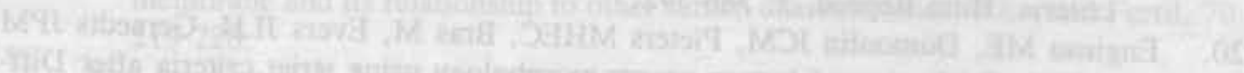

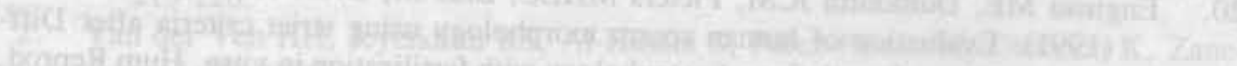

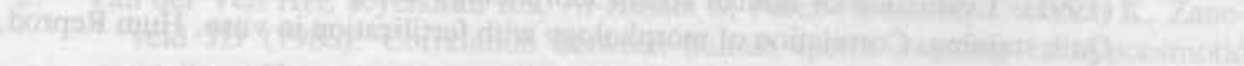

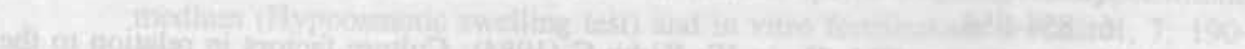

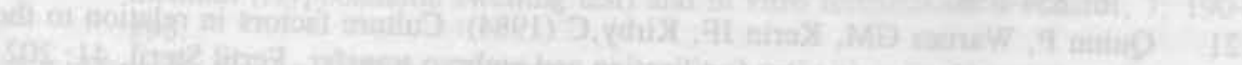
405.

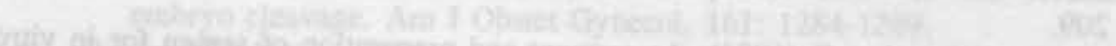

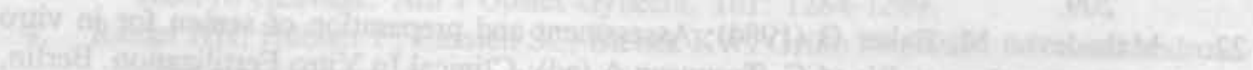

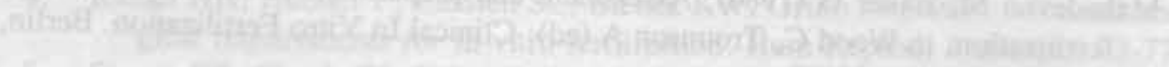

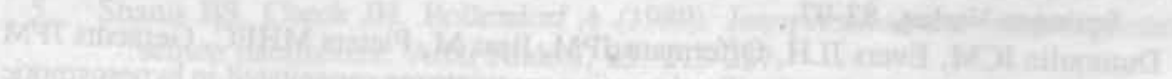
Thing

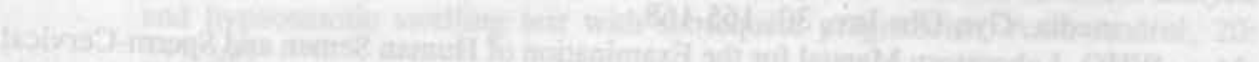

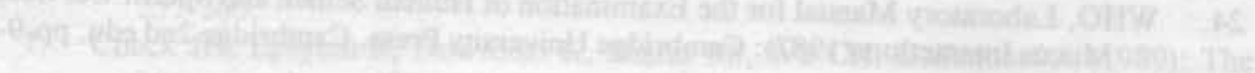

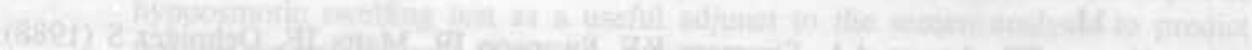
thar

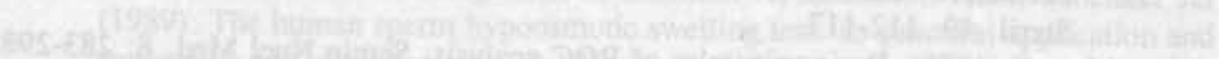

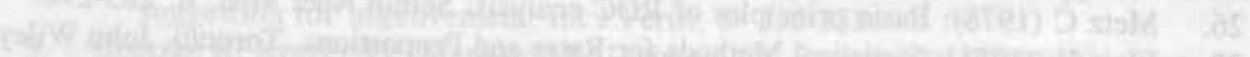
-

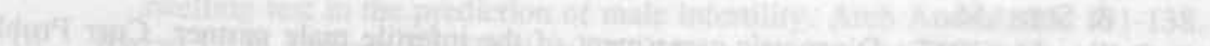
-

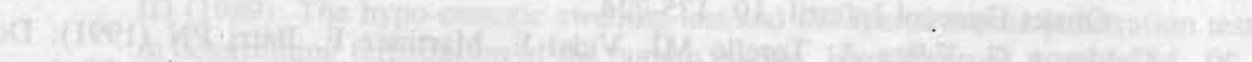

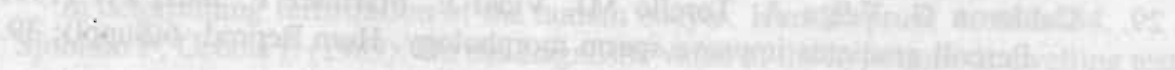

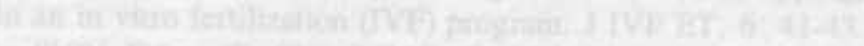

Q⿻十

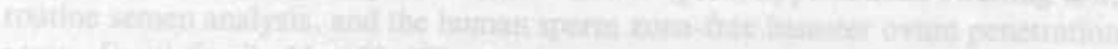

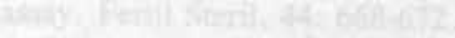

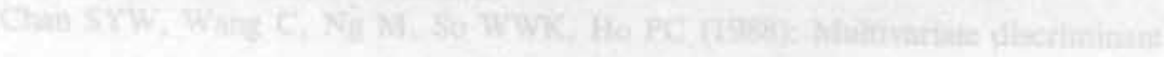




\title{
Male factor as determinant of in vitro fertilization outcome
}

\author{
M.E. Enginsu, M.H.E.C. Pieters, J.C.M. Dumoulin, J.L.H. Evers, \\ J.P.M. Geraedts. Human Reproduction 7, 1136-1140, 1992.
}

The effect of different semen parameters was evaluated in 200 consecutive couples in an in vitro fertilization (IVF) program. All semen analyses were performed on the native aliquot of semen which was subsequently prepared and used for in vitro insemination. Morphology evaluation using strict criteria (kappa 0.46 and $r=0.565$ ) was compared with progressive motile sperm density (kappa 0.37 and $r=0.333$ ) and the conventional World Health Organization (WHO) evaluation of morphology (kappa 0.31 and $r=0.378)$. Results show that morphology evaluation using strict criteria is the best predictor of IVF and progressive motile sperm density can be an optional method. The combined results of strict morphology and progressive motile sperm density show that if both parameters were below the cut-off points of $5 \%$ and $3 x$ $10^{6} / \mathrm{ml}$ respectively, the fertilization rate per oocyte was very low (18\%). No pregnancies were achieved in this group. When both parameters were above the cut-off points, the fertilization rate per oocyte was high $(72 \%)(P<0.00005)$ and pregnancy rate per embryo transfer was $27 \%$. Predictive values indicate that morphology evaluation using strict criteria and progressive motile sperm density can be used as a patient selection criteria for infertility clinics.

\section{Introduction}

Detection of reduced male fertility among participants in an in-vitro fertilization (IVF) program is important. It is necessary to try to predict the outcome before accepting couples for artificial reproduction. Sperm density, motility and percentage with normal morphology have been the traditional criteria to assess semen quality (Menkveld et al., 1990).

The importance of semen analysis in IVF programs has been studied by several groups (Aitken et al., 1982; Comhaire et al., 1988; Kruger et al., 1988b; Liu et al., 1988). After the first description of normal sperm morphology using strict criteria (Kruger et al., 1988a; and Menkveld et al., 1990), the role of sperm morphology received renewed attention. In a previous study, we compared the strict evaluation method with the conventional analysis and demonstrated the superiority of the new technique in predicting fertilization in 
vitro (Enginsu et al., 1991). Semen samples with a concentration $<20 \times 10^{6} / \mathrm{ml}$ and/or progressive motility $<30 \%$ were excluded in that study in order to study the contribution of morphology per se.

The aim of the present study thus was to estimate the prognostic value of semen analysis in a larger unselected population. We decided to correlate semen analysis the fertilization rates, embryo transfer rate and the pregnancy rates in all IVF cases. Thus, none of the semen samples with oligo-, astheno- or teratozoo-spermia, nor any combination of abnormalities were excluded. This also allowed us to evaluate the contribution of the other conventional semen parameters.

\section{Materials and Methods}

\section{Patients}

Two-hundred consecutive first treatment cycles were evaluated in IVF couples between September 1989 and November 1991. The couples were introduced to the IVF program for one of the following indications: tubal pathology $(n=102)$, unexplained infertility $(n=63)$, endometriosis $(n=16)$, male subfertility $(n=12)$, immunological infertility $(n=4)$ or polycystic ovary syndrome $(n=3)$.

\section{In vitro fertilization}

Multiple follicular development was induced using a mild stimulation protocol with human menopausal gonadotropin (hMG, Humegon; Organon, Oss, The Netherlands) $225 \mathrm{IU}$ i.m. daily starting on day 3 during pituitary downregulation with buserelin (Suprefact, Hoechst Amsterdam, The Netherlands). The growth of the follicles was followed ultrasonographically on day 3 , day 8 and daily from day 10 onwards. Human chorionic gonadotropin (hCG, Pregnyl; Organon) 10.000 IU was given i.m. on the evening of the day the mean diameter of the dominant follicle reached $\geq 18 \mathrm{~mm}$. Oocyte recovery was performed by vaginal ultrasound-guided aspiration 34 hours after hCG injection. Whittingham's T6 medium (Quinn et al., 1984) supplemented with $10 \%$ heatinactivated maternal serum was used for the culture of oocytes and embryos and for washing and incubation of the spermatozoa. Oocytes were cultured in an atmosphere of $5 \% \mathrm{CO}_{2}$ in air, which resulted in $\mathrm{pH} 7.4$ in the culture medium after equilibration. The osmolality of this medium was adjusted to $285 \mathrm{mOs} / \mathrm{kg}$. Semen was produced $\approx 2 \mathrm{~h}$ after the ovum retrieval. For each sample, a suspension of motile spermatozoa was prepared by a swim-up technique (Mahadevan and Baker, 1984) and incubated for another $2 \mathrm{~h}$. At $5 \mathrm{~h}$ after oocyte recovery, $\approx 50.000$ motile spermatozoa were added to each oocyte. After incubation for $18-20 \mathrm{~h}$, the oocytes were checked for the presence of 
pronuclei as evidence of fertilization. Fertilized oocytes were transferred to fresh medium and cultured for another $24 \mathrm{~h}$. At $42-44 \mathrm{~h}$ after insemination, $\leq 4$ embryos with the best morphological appearance were transferred to the uterus of the patient; any supernumerary oocytes were frozen.

\section{Semen Handling}

Conventional methods were used to evaluate the volume, sperm density and motility. Two groups of motile sperm were recognized: slow, sluggish, movement or movement of the spermatozoa hampered by a clearly visible morphological defect (group A) and rapid and linear progressive movement (group B). The total number of the spermatozoa in group B was defined as the progressive motile sperm density (PMSD). Conventional morphology of spermatozoa was evaluated according to the guidelines of the World Health Organization (WHO, 1987).

Morphology evaluation using strict criteria (MEUSC) was performed by using two smears from each semen sample. The slides used for semen smears were previously thoroughly cleaned with $100 \%$ methanol. In order to obtain as thin a smear as possible only $5 \mu \mathrm{l}$ of semen were used. The slides were air-dried for $\approx 3$ minutes, fixed for $15 \mathrm{~s}$ in Diff-Quik fixative $(1.8 \mathrm{mg} / \mathrm{L}$ triarylmethane in methyl alcohol) prior to staining with Diff-Quik solution $1(1 \mathrm{~g} / \mathrm{L}$ xanthene in sodium azide-preserved buffer) for $10 \mathrm{~s}$ and with Diff-Quik solution $2(0.625$ $\mathrm{g} / \mathrm{L}$ azure $\mathrm{A}$ and $0.625 \mathrm{~g} / \mathrm{L}$ methylene blue in buffer) for $5 \mathrm{~s}$. Diff-Quik solutions were obtained from Baxter, Utrecht, The Netherlands. Between the fixing step and each of the staining steps, the excess solutions were drained from the slides by placing them vertically on bibulous paper. The morphology was evaluated as detailed by Kruger et al.(1988a). At least 200 spermatozoa per patient were evaluated at a magnification of $\mathrm{x} 1000$.

\section{Statistics}

Correlations between individual test results and fertilization rates were evaluated by calculating Pearson's rank correlation coefficient.

Patients were divided into two groups according to whether or not fertilization had occurred. Receiver operating characteristic (ROC) curves as described by Metz (1978) were constructed for the assessment of the effectiveness of each individual parameter in predicting fertilization. The curves were constructed from the cumulative frequency distribution of a particular semen characteristic in the two populations by plotting the proportions of subjects in the first group with values less than a given value $\mathrm{x}$ against the proportion in the second group with values less than $x$ (Comhaire et al., 1988). After constructing the ROC curves, it is important to make further calculations to decide on a clinically acceptable cut-off point. Therefore, Cohen's kappa statistics (Fleis JL, 1981) 
were calculated to assess the agreement between each diagnostic test and fertilization, after correction for agreement by chance for the best cut-off point. Sensitivity and specificity are often used to determine the overall quality of diagnostic tests compared with other similar tests. In the context of clinical practice, the predictive value of the test was thought to be more important than its distribution among fertile and infertile individuals; likelihood ratios are more direct expressions of the test quality because they separately quantify the performance of abnormal and normal test results. Therefore, likelihood ratios (LR) were calculated (Collins, 1987) to express the likelihood of fertilization over the likelihood of non-fertilization, given normal LR + or abnormal LR- test results.

The relationships between progressive motile sperm density and the percentages of normal morphology with fertilization rates per oocyte and embryo transfer rate per patient were examined using a chi-square test in a $2 \times 2$ table. The distribution of failed fertilization $(0 \%)$ and poor fertilization $(<25 \%)$ rates were evaluated in four different categories: strict criteria (MEUSC) with progressive motile sperm density (PMSD) respectively of I, $<5 \%$ and $<3 \mathrm{million} / \mathrm{ml}$, II, $<5 \%$ and $>3$ million/ml, III, $>5 \%$ and $<3$ million/ml and IV, $>5 \%$ and $>3$ million $/ \mathrm{ml}$.

Figure 1. Receiver operating characteristic (ROC) curves of semen parameters.

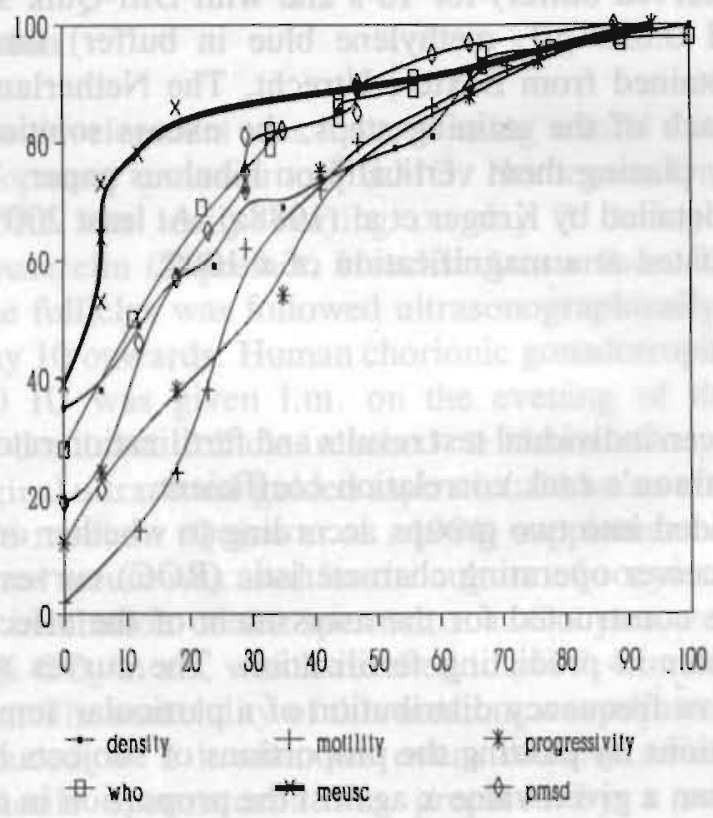




\section{Results}

Fertilization rates, embryo transfer rates and pregnancy rates were calculated for the different sperm categories and ROC curves were constructed. The curve of MEUSC enclosed a larger area (below and to the right) than the other parameters (Figure 1).

Table I. Comparison of different parameters of semen samples on the basis of correlation with fertilization in vitro (Cohen's kappa, likelihood ratios (LR), Pearson correlation coefficient).

\begin{tabular}{lrrrrrr}
\hline Parameter & Cut-off & kappa & LR + & LR & r & P \\
\cline { 2 - 7 } Volume (ml) & 3 & 0.02 & 1.3 & 0.90 & -0.10 & N.S. \\
Sperm density (x 10\% $/ \mathrm{ml}$ ) & 20 & 0.27 & 1.4 & 0.23 & 0.309 & 0.001 \\
Motility (\%), group A & 30 & 0.22 & 1.3 & 0.24 & 0.177 & N.S. \\
Progressive motility (\%), group B & 30 & 0.18 & 1.2 & 0.25 & 0.269 & 0.001 \\
PMSD (x 10\%l) & 3 & 0.37 & 1.5 & 0.12 & 0.333 & 0.001 \\
Morphology (\%) (conventional) & 14 & 0.31 & 1.6 & 0.20 & 0.378 & 0.001 \\
MEUSC (\%) & 4 & 0.46 & 4.7 & 0.17 & 0.565 & 0.001 \\
\hline
\end{tabular}

$\mathrm{r}$ and $\mathrm{P}=$ Pearson's correlation coefficient and probability; kappa $=$ Cohen's kappa $(<0.4$ $=$ poor agreement; $0.4-0.75=$ good agreement); $L R+=$ Likelihood of fertilization, given normal test result ( $<2=$ inoperative; $2-5$ fair agreement; $5-10$ good agreement); LR- = Likelihood of fertilization, given abnormal test result $(0.1-0.2=$ good agreement; $0.2-0.5$ $=$ fair agreement; $>0.5=$ inoperative)

Table II. Combined results of morphology evaluation using strict criteria (MEUSC) and progressive motile sperm density (PMSD) in prediction of fertilization in vitro.

\begin{tabular}{lrrrrr} 
Category & n Oocytes* & Fert(\%)* & \multicolumn{2}{c}{ ET(\%)* Pregnancy (\%)* } \\
\hline I MEUSC <5 and PMSD $<3$ & 10 & 103 & $19(18 \%)^{\mathrm{a}}$ & $4(40 \%)^{\mathrm{c}}$ & $0(0 \%)$ \\
II MEUSC $<5$ and PMSD $>3$ & 29 & 303 & $86(28 \%)^{\mathrm{b}}$ & $20(69 \%)^{\mathrm{f}}$ & $2(10 \%)$ \\
III MEUSC $>5$ and PMSD $<3$ & 7 & 59 & $27(46 \%)^{\mathrm{c}}$ & $5(71 \%)^{\mathrm{k}}$ & $1(20 \%)$ \\
IV MEUSC $>5$ and PMSD $>3$ & 154 & 1682 & $1213(72 \%)^{\mathrm{d}}$ & $149(98 \%)^{\mathrm{h}}$ & $40(27 \%)$ \\
\hline
\end{tabular}

*Oocytes: number of oocytes collected, Fert: number of oocytes fertilized, ET: number of patients that reached to the embryo transfer stage, Pregnancy: number of patients who became pregnant after embryo transfer; a-h Within the columns the significant differences are as follows: There were no significant differences between $a$ and $b$; Between $a$ and $c$ : $P<0.0005$, a and $d: P<0.000001, b$ and $c: P<0.01, b$ and $d: P<0.000001, c$ and $d:$ $P<0.00005$; There were no significant differences between $e$ and $f, e$ and $g$ and $f$ and $g$; Between $\mathrm{e}$ and $\mathrm{h}: \mathrm{P}<0.000001, \mathrm{f}$ and $\mathrm{h}: \mathrm{P}<0.000001, \mathrm{~g}$ and $\mathrm{h}: \mathrm{P}<0.05$.

The best cut-off points of the various sperm parameters were determined by the 
kappa values and the likelihood ratios were calculated (Table I). Also Pearson's correlation coefficients were determined for the relationships between the separate sperm parameters and fertilization in vitro (Table I).

The only parameter with a kappa value high enough to be of clinical significance (0.46) was sperm morphology using strict criteria. Although not reaching the level of 0.40 , the progressive motile sperm density was the second best $(0.37)$. The superiority of the strict criteria (kappa 0.43 ) over WHO criteria (Kappa 0.31) was clear.

As a second best predictor progressive motile sperm density (PMSD) was evaluated further and IVF results were combined with the data on strict criteria (MEUSC)(Table II). When both parameters were below their respective cut-off points (MEUSC $<5 \%$ and PMSD $<3 \times 10^{6} / \mathrm{ml}$; category I), the fertilization rate per oocyte was only $18 \%$. If the MEUSC score was below and the PMSD was above the cut-off point (category II), the fertilization rate was $28 \%$. This was significantly different from categories III and IV $(\mathrm{P}<0.01$ and $\mathrm{P}<-$ 0.000001 ). When the MEUSC score was above and PMSD was below the respective cut-off points (category III), the fertilization rate was $46 \%$ and significantly different from the other three groups $(\mathrm{P}<0.0005, \mathrm{P}<0.05$ and $\mathrm{P}<0.00005$ ). Finally, when both parameters were above their respective cut-off point (category IV) the fertilization rate was $72 \%(\mathrm{P}<0.000001, \mathrm{P}<0.000001$, $\mathrm{P}<0.00005$ ).

The number of patients achieving embryo transfer increased from $40 \%$ in category I to $98 \%$ in category IV and only category IV was significantly different from the other three categories $(\mathrm{P}<0.000001, \mathrm{P}<0.000001$, $\mathrm{P}<0.05)($ Table II).

Table III. Distribution of different fertilization rates.

Category n $\quad$ Fertilization rate per oocyte

\begin{tabular}{|c|c|c|c|c|c|c|}
\hline \multicolumn{2}{|c|}{ 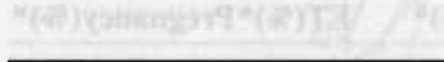 } & \multicolumn{2}{|c|}{ (Number of IVF procedures) } & \multicolumn{2}{|r|}{$0 \%$} & $<25 \%$ \\
\hline I & MEUSC $<5$ and $\mathrm{PMSD}<3$ & 10 & & 6 & $(60 \%)$ & $7(70 \%)$ \\
\hline II & MEUSC $<5$ and PMSD $>3$ & 29 & & 8 & $(28 \%)$ & $16(55 \%)$ \\
\hline III & MEUSC $>5$ and $\mathrm{PMSD}<3$ & 7 & & 1 & $(14 \%)$ & $2(28 \%)$ \\
\hline IV & MEUSC $>5$ and PMSD $>3$ & 154 & & 2 & $(1 \%)$ & $5(3 \%)$ \\
\hline
\end{tabular}

The pregnancy rate increased from $0 \%$ in category $I$ to $27 \%$ in category IV. The number of IVF procedures resulting in $0 \%$ and $<25 \%$ fertilization rate respectively, was highest ( 60 and $70 \%$ respectively) in category I and was lowest in category IV, (1\% and $3 \%$ respectively). The percentage of patients who achieved fertilization and who had a positive test (above cut-off points) was $98 \%(158 / 161)$ for strict criteria (2\% false positive) and $95 \%(173 / 183)$ for the progressive motile sperm density (5\% false positive)(Table III). 


\section{Discussion}

This study shows the potential of using IVF results for studying the predictive value of sperm tests. Of the usual seminal measurements made in practice, the best cut-off points to predict fertilization failure were found to be: sperm density $20 \times 10^{6} / \mathrm{ml}$, motility $30 \%$, progressive motility $30 \%$, progressive motile sperm density $3 \times 10^{6} / \mathrm{ml}$, normal morphology using conventional WHO criteria $14 \%$, and morphology evaluation using strict criteria $4 \%$. The cut-off point we found for the WHO criteria is below the official $50 \%$ WHO limits. Since, this variable was only studied in relation to IVF results in our study, it is therefore only applicable to IVF conditions. Subsequent treatment of male and female gametes before insemination might be an explanation for the difference in cut-off points.

The technique of morphology evaluation using strict criteria has been discussed in previous articles (Enginsu et al., 1991, 1992). The present study, in an unselected group of subjects, clearly shows that this new strict technique for evaluation of human sperm morphology plays an important role in predicting IVF results. Furthermore, it is clear that this method is superior to WHO criteria for human sperm evaluation in vitro.

The role of semen quality in an IVF programme has been shown before (Rogers et al., 1983; Acosta et al., 1988; Chan et al., 1988; Comhaire et al., 1988; Kruger et al., 1988b; Liu et al., 1988; Dunphy et al., 1989; Turner et al., 1989; Enginsu et al., 1991). Semen parameters are usually evaluated separately and the combined effect of individual parameters has rarely been studied.

Glazener et al. (1987) used different combinations of semen parameters and found the motile normal sperm density to be the best combination, but inferior to the post-coital test. The study was done in an in-vivo fertilization group. Their cut-off value of $4 \times 106 / \mathrm{ml}$ motile normal sperm density is close to the value of $3 \times 106 / \mathrm{ml}$ of progressive motile sperm density which we found. In another study $5 \times 106 / \mathrm{ml}$ motile spermatozoa was taken as a cut-off point by Francavilla et al., (1990). In that study the effect of semen quality was evaluated on the basis of the pregnancy outcomes after intra-uterine insemination. They also divided the semen parameters in four categories and there were no pregnancies in the group where normal morphology (WHO criteria) was $<50 \%$ and motile sperm count was $<5 \times 10^{6} / \mathrm{ml}$. Comhaire et al. (1988) also studied the predictive value of individual and combined semen parameters. They also stated that sperm morphology, especially head morphology, is the best discriminant in vitro. However, it is surprising to see that $5 \%$ morphologically normal forms were their cut-off point although the morphology evaluation was carried out by conventional WHO criteria. These studies show that the combined application of different parameters offers clinically significant predictive values for fertilization in vitro.

It is important to define the infertile group before starting to calculate the ROC 
curves. We regard all patients with no fertilization as infertile. However absence of fertilization maybe due to all sorts of technica! problems which ultimately affect, and these should be excluded. Therefore, the distribution of attempts with no fertilization and poor fertilization was evaluated (Table III). It can clearly be seen that both of these fertilization groups $(0 \%$ and $<25 \%)$ had the highest rates in category I where semen parameters were very poor and lowest in category IV where semen parameters were very good. This distribution and the fact that all the IVF treatments were carried out with a standard protocol leaves no doubt that the poor fertilization rates were indeed a consequence of male factors.

Semen samples show large variations over time, mainly with respect to sperm density, volume and percentage motility (Mallidis et al., 1991). Therefore, it is important to ascertain the current status of the male partner before the IVF treatment. Since the correlation between the semen parameters and the IVF outcome is clear, it can be suggested that before IVF treatment commences, semen samples from the male partners should be evaluated for morphology evaluation using strict criteria and the of progressive motile sperm density. In our hands, these two parameters are also reliable on the basis of previous semen analysis (Enginsu et al., 1992).

Application of these techniques during infertility evaluation may allow clinicians to determine whether or not the couple should be excluded from IVF treatment on the basis of male factor test results.

This study shows that category I patients are the ones with the poorest results. Although it is highly probable that the semen from this group of patients will fail to fertilize the oocytes from their partners, occasionally fertilization may occur. The embryo transfer rate is also decreased, and, in the present study, no pregnancies were achieved. Accordingly, exclusion of these couples from IVF treatment should be considered.

\section{References}

Acosta AA, Oehninger S, Morshedi M, Swanson RJ, Scott R, Irianni F, (1988): Assisted reproduction in the diagnosis and treatment of the male factor. Obstet Gynecol Survey, 44: $1-18$

Aitken RJ. Best FSM, Richardson DW, Djahanbakhch O, Mortimer D, Tempelton AA, Lees MM (1982): An analysis of sperm function in cases of unexplained infertility: conventional criteria, movement characteristics, and fertilizing capacity. Fertil Steril, 38: 212-221

Chan SYW, Wang C, Chan STH, Ho PC, So WWK, Chan YF, Ma HK (1988): Predictive value of sperm morphology and movement characteristics in the outcome of in vitro fertilization of human oocytes. J IVF ET, 6: 142-148

Collins JA (1987): Diagnostic assessment of the infertile male partner. Curr Probl Obstet Gynecol Infertil, 10: 175-224

Comhaire FH, Vermeulen L, Hinting A, Schoonjans F (1988): Accuracy of sperm characteristics in predicting the in vitro fertilizing capacity of semen. J IVF ET, 5: 326-331

Dunphy BC, Neal LM, Cooke ED (1989): The clinical value of conventional semen analysis. 
Fertil Steril, 51: 324-329

Enginsu ME, Dumoulin JCM, Pieters MHEC, Bras M, Evers JLH, Geraedts JPM(1991): Evaluation of human sperm morphology using strict criteria after Diff-Quik staining: Correlation of morphology with fertilization in vitro. Hum Reprod, 6: 854-858

Enginsu ME, Dumoulin JCM, Pieters MHEC, Bergers M, Evers JLH, Geraedts JPM (1992): Comparison between the hypoosmotic swelling test and morphology evaluation using strict criteria in predicting fertilization in vitro. J Ass Reprod Genet, 9: 259-264

Fleis JL (1981): Statistical Methods for Rates and Proportions. Toronto, John Wiley \& Sons , 56

Francavilla F, Romano R, Santucci R, Poccia G (1990): Effect of sperm morphology and motile sperm count on outcome of intrauterine insemination in oligospermia and/or asthenozoospermia. Fertil Steril, 53: 892-897

Glazener CMA, Kelly NJ, Weir MJA, David JSE, Cornes JS, Hull MGR (1987): The diagnosis of male infertility-prospective time specific study of conception rates related to seminal analysis and post-coital sperm-mucus penetration and survival in otherwise unexplained infertility. Hum Reprod, 2: 665-671

Kruger TF, Acosta AA, Simmons KF, Swanson JR, Matta J, Oehniger S (1988a): Predictive value of abnormal sperm morphology in in vitro fertilization. Fertil Steril, 49: 112-117

Kruger TF, Swanson RJ, Hamilton M, Simmons KF, Acosta AA, Matta JF, Oehniger S, Morshedi M (1988b): Abnormal sperm morphology and other semen parameters related to the outcome of the hamster oocyte human sperm penetration assay. Int J Androl, 11: $107-113$

Liu DY, Du-Plessis YPD, Nayudu PL, Johnston WIH, Baker HWG (1988): The use of in vitro fertilization to evaluate putative tests of human sperm function. Fertil Steril, 49: 272-277

Mahadevan M, Baker G (1984): Assessment and preparation of semen for in vitro fertilization; in Wood C, Trounson A (ed): Clinical In Vitro Fertilization. Berlin, Springer-Verlag, 83-97

Mallidis C, Howard EJ, Baker HWG (1991): Variation of semen quality in normal men. Int J Androl, 14: 99-107

Menkveld,R, Stander,FSH, Kotze TJW, Kruger TF, Van Zyl JA (1990): The evaluation of morphological characteristics of human spermatozoa according to stricter criteria. Human Reprod, 5: 586-592

Metz C (1978): Basic principles of ROC analysis. Semin Nucl Med, 8: 283-298

Quinn P, Warnes GM, Kerin JF, Kirby C (1984): Culture factors in relation to the success of human in vitro fertilization and embryo transfer. Fertil Steril, 41: 202-209

Rogers BJ, Bentwood BJ, Van Campen H, Helmbrecht G, Sodendahl D, Hale RW (1983): Sperm morphology assessment as an indicator of human fertilizing capacity. J. Androl, 4: $119-125$

Turner TW, Schrader SM, Perez-Pelaez M, Karuhn RF, Van der Ven HH, Jeyendran RS (1989): Morphometric and volumetric comparisons of human spermatozoa. Arch Androl, 23: 201-206

WHO, Laboratory Manual for the Examination of Human Semen and Sperm-Cervical Mucus Interactions (1987): Cambridge University Press, Cambridge 2nd edn, pp.9-11 
-

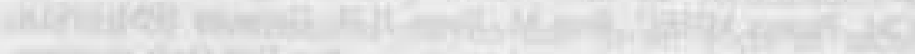

-

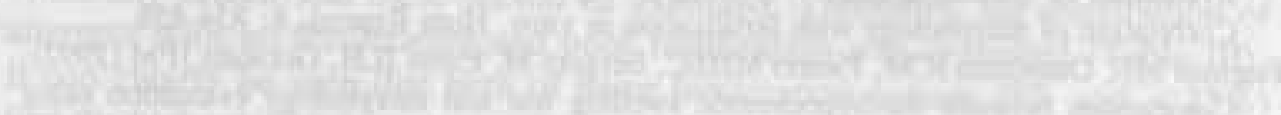
15.

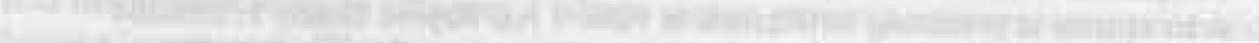
C. 1)

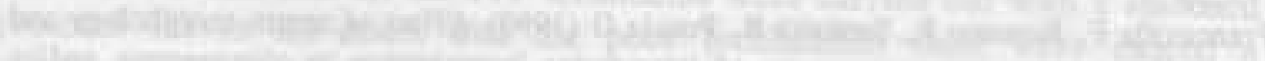

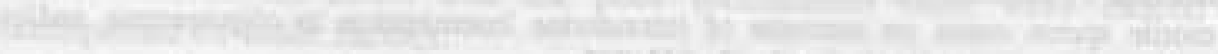

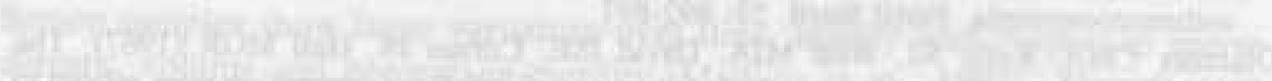

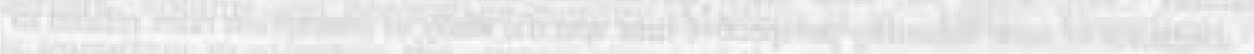

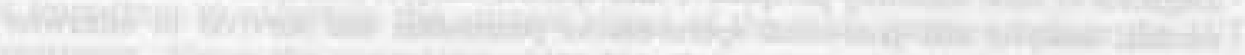

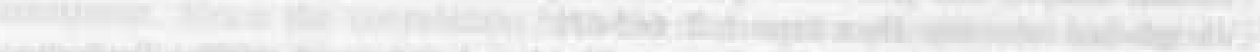

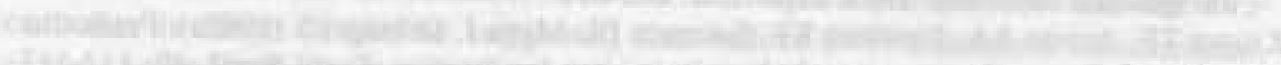

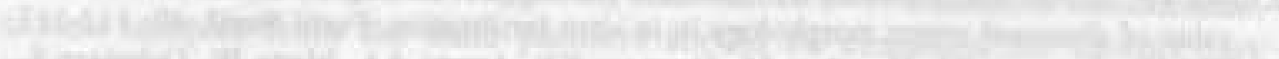

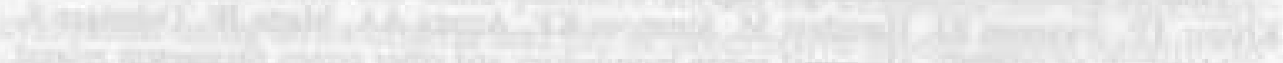

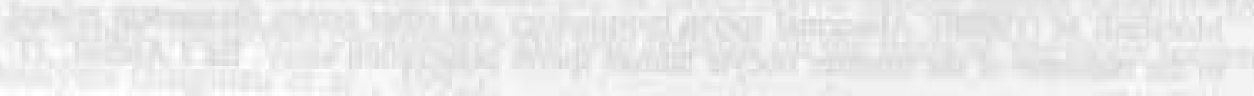

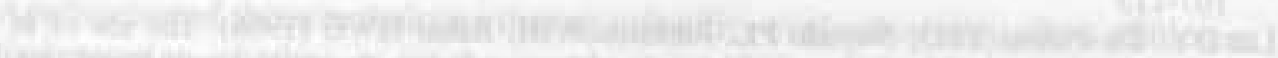

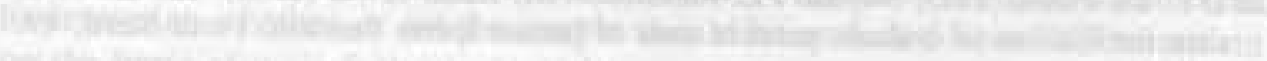

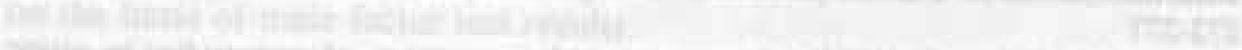

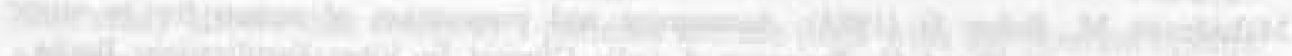

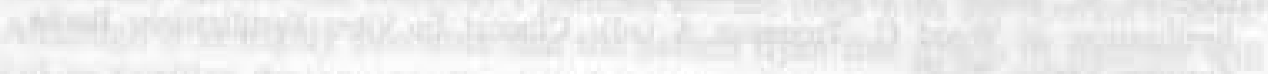

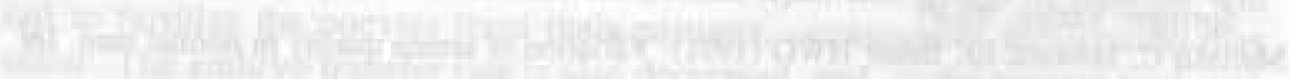

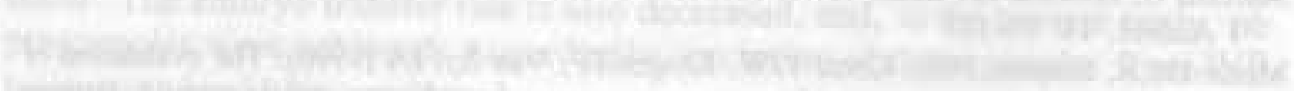

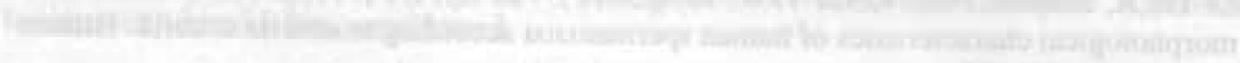

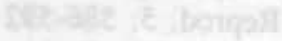

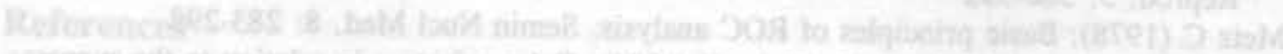

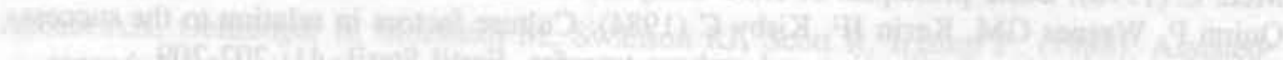

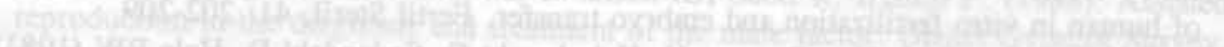

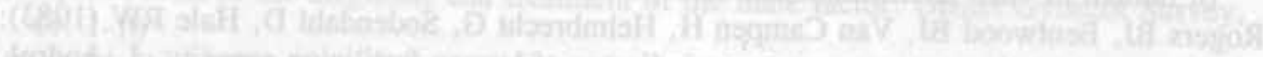

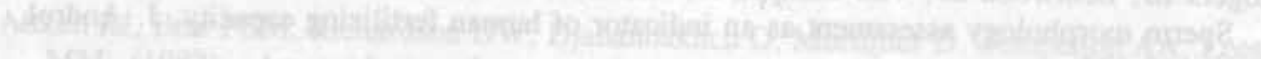

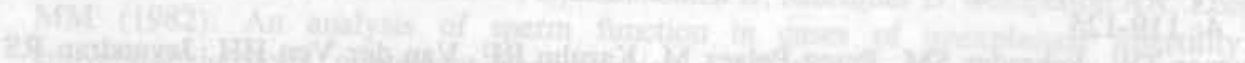

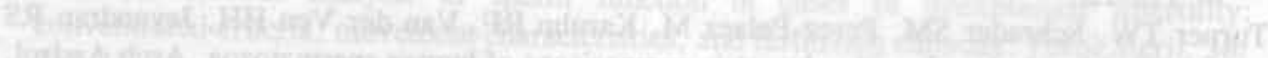

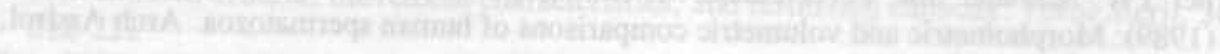

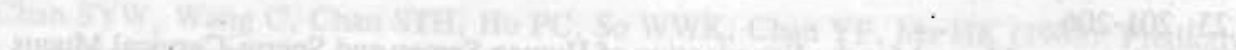

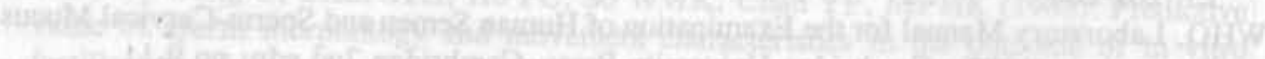

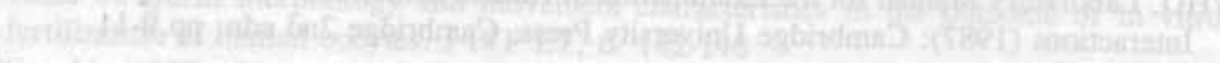

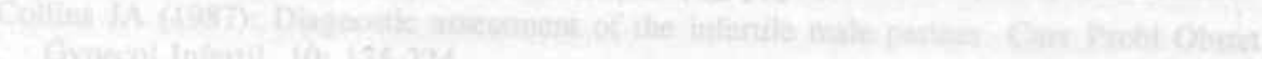

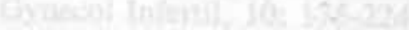

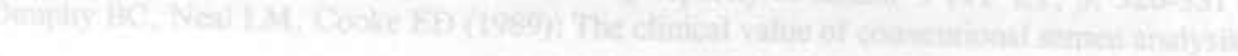




\title{
Predictive value of morphologically normal sperm concentration in the medium for in vitro fertilization
}

\author{
M.E. Enginsu, J.C.M. Dumoulin, M.H.E.C. Pieters, J.L.H. Evers, J.P.M. \\ Geraedts. International Journal of Andrology 16, 113-120, 1993.
}

Morphology evaluation of spermatozoa using strict criteria (MEUSC) and conventional sperm parameters were studied with respect to fertilization and pregnancy outcome before and after a swim-up procedure. Recovered oocytes were inseminated with 50.000 progressive motile spermatozoa, and this study assess the influence of the total number of spermatozoa and of the percentage with strictly normal morphology in the insemination sample on the outcome of IVF. The results showed that the percentages of spermatozoa with normal morphology using strict criteria, both in native and in post-swim-up samples, were the best predictors of IVF outcome. Their respective cutoff points were 5\% and $8 \%$. The number of morphologically normal spermatozoa in the insemination medium also showed a good correlation with fertilization. However, it was not possible to find a proper cut-off point for this parameter. The patients were categorized on the basis of their native and post-swim-up morphology scores. Category 1 , in which both parameters were below their respective cut-off points, showed a $7 \%$ fertilization rate and a $0 \%$ pregnancy rate. Category 3 , in which both parameters above their cut-off points, showed a $70 \%$ fertilization and a $23 \%$ pregnancy rate. This suggests that sperm morphology can be used as a criterion for patient selection for IVF as an aid to identification of possibly subfertile males.

\section{Introduction}

Semen quality, plays an important role in human in vitro fertilization (IVF). Three basic parameters determine sperm quality: morphology, progressive motility and density. After the introduction of strict criteria for the evaluation of sperm morphology by Kruger et al.(1986), the percentage of morphologically normal spermatozoa has acquired more attention than previously. We compared strict criteria with the conventional World Health Organization (WHO) criteria both in a selected (Enginsu et al., 1991) and an unselected (Enginsu et al., 1992a, Enginsu et al., 1992b) groups of IVF couples and found that the strict criteria were superior to the WHO criteria in predicting fertilization. It has also been shown previously that when different parameters such as morphology, progressive motility and density are combined, the predictive value with respect to fertilization increases (Glazener et al., 1987 ,Francavilla et al., 1990, 
Enginsu et al., 1992b).

Sperm swim-up is a selection procedure used widely to select spermatozoa for insemination. Previous studies have shown that 50.000 progressively motile spermatozoa per $\mathrm{ml}$ is the optimum for in vitro fertilization (Diamond et al., 1985, Dumoulin et al., 1991, Dumoulin et al., 1992, Wolf et al., 1984). Dumoulin et al. (1992) have also shown that when human oocytes are inseminated with $>50.000$ progressive motile spermatozoa per $\mathrm{ml}$, subsequent embryonic development is retarded.

In our IVF protocol human oocytes are inseminated with 50.000 per $\mathrm{ml}$ of progressively motile spermatozoa after a swim-up procedure. This allowed us to evaluate the role of morphologically normal spermatozoa in the IVF medium. The aims of the present study were: 1) to investigate the relation between the native and post-swim-up samples, 2) to determine a clinically acceptable cut-off point with respect to morphologically normal spermatozoa evaluated with strict criteria in native and post-swim-up samples; and 3) to evaluate the effect of the density of morphologically normal spermatozoa in the insemination medium with respect to fertilization in vitro.

\section{Materials and Methods}

\section{Patients}

A total number of 185 subsequent first treatment cycles was evaluated in IVF couples between March 1990 and February 1992. The couples were introduced into the IVF program for one of the following indications: tubal pathology (99), unexplained infertility (64), endometriosis (12), male subfertility (6), immunological infertility (2), polycystic ovary syndrome (2).

\section{In vitro fertilization}

Multiple follicular development was induced using a mild stimulation protocol with $225 \mathrm{U}$ human menopausal gonadotropin (hMG, Humegon; Organon, Oss, The Netherlands) administered daily by intramuscular injection starting on day 3 after induction of pituitary down- regulation using buserelin (Suprefact, Hoechst BV, Amsterdam, The Netherlands). Growth of the follicles was followed using ultrasonography on days 3 and 8 and daily from day 10 onwards. Then $10.000 \mathrm{U}$ human chorionic gonadotrophin (hCG, Pregnyl; Organon) was administered intramuscularly on the evening of the day when the mean diameter of the largest follicle reached $\geq 18 \mathrm{~mm}$. Oocytes were recovered by aspiration guided by vaginal ultrasound at $34 \mathrm{~h}$ after hCG injection.

Whittingham's T6 medium (Quinn et al., 1984) supplemented with $10 \%$ heatinactivated maternal serum was used for the culture of oocytes and embryos, 
and for washing and incubation of the spermatozoa. Oocytes were cultured in an atmosphere of $5 \% \mathrm{CO}_{2}$ in air, which resulted in a $\mathrm{pH}$ of 7.4 in the culture medium after equilibration. The osmolality of this medium was adjusted to 285 $\mathrm{mOs} \mathrm{kg}^{-1}$.

Semen was produced approximately $2 \mathrm{~h}$ after the oocyte retrieval. For each sample, a suspension of motile spermatozoa was prepared by a swim-up technique (Mahadevan and Baker, 1984) and incubated for a further $2 \mathrm{~h}$. At 5 $\mathrm{h}$ after oocyte recovery, 50.000 progressively motile spermatozoa were added per ml. After incubation for $18-20 \mathrm{~h}$, the oocytes were checked for the presence of pronuclei as proof of fertilization. The fertilized oocytes were then transferred to fresh medium and cultured for a further $24 \mathrm{~h}$. At $42-44 \mathrm{~h}$ after insemination, up to four embryos with the best morphology were transferred to the uterus of the patient; any supernumerary embryos were frozen.

\section{Semen Handling}

Conventional methods were used to evaluate the volume, sperm density and motility (WHO, 1987). Motility of spermatozoa was also determined according to the guidelines of WHO except that only two groups of motile spermatozoa were distinguished: slow, sluggish movement or movement of the spermatozoa hampered by an obvious, clearly visible morphological defect (group A) and rapid and linearly progressive movement (group B). The total number of the spermatozoa in group B was defined as the progressive motile sperm density (PMSD).

Morphology evaluation using strict criteria (MEUSC) was performed as described previously (Enginsu et al., 1992b). Briefly, two smears from each semen sample were prepared. The slides were air-dried for approximately 3 $\mathrm{min}$, fixed for $15 \mathrm{~s}$ in Diff-Quik fixative prior to staining with Diff-Quik solution 1 for $10 \mathrm{~s}$ and with Diff-Quik solution 2 for $5 \mathrm{~s}$ Diff-Quik solutions were obtained from Baxter, Utrecht, The Netherlands. In between the fixing step and each of the staining steps, the excess solutions were drained from the slides by placing them vertically on absorbent paper. Sperm morphology was evaluated as outlined in detail by Kruger et al. (1986). At least 200 spermatozoa per patient were evaluated at a magnification of $x 1000$.

The count, percentage motility, percentage progressive motility and progressive motile sperm density in the swim-up portions were assessed before insemination in order to adjust the final concentration to 50.000 progressively motile spermatozoa per $\mathrm{ml}$. After insemination, the rest of the sample was used for evaluation of sperm morphology. Since the final concentration of the inseminated spermatozoa was low (as expected after any kind of sperm preparation) the solution was centrifugated for $10 \mathrm{~min}$. The pellet was then suspended in $0.2 \mathrm{ml}$ medium and two smears from this solution were stained as described previously and used for evaluation of sperm morphology. The number of morphologically 
normal spermatozoa used for IVF (IMNS) was then calculated for each sample as follows:
IMNS = SDSU $\times$ MEUSC $\times$ IVO, where
SDSU = Sperm density of the swim-up fraction (million $/ \mathrm{ml}$ ),
MEUSC = Percentage of morphologically normal sperm in IVF medium, and
IVO $\quad=$ Volume of IVF medium $(\mu \mathrm{l})$.

\section{Statistics}

Patients were divided into two groups according to whether or not fertilization had occurred. Receiver operating characteristic (ROC) curves as described by Metz (1978) were constructed for assessment of the effectiveness of each individual parameter in predicting fertilization. After constructing the ROC curves, it is important to calculate a clinically acceptable cut-off point. Therefore, Cohen's Kappa statistics (Fleis, 1981) were calculated to assess the agreement between each diagnostic test and fertilization, after correction for agreement by chance for the best cut-off point.

Sensitivity and specificity are often used to determine the overall quality of diagnostic tests compared with other similar tests. In the context of clinical practice, predictive value is more important than its distribution among fertile and infertile individuals. Likelihood ratios are more direct expressions of test quality because they separately quantify the clinical significance of abnormal and normal test results. Therefore, likelihood ratios were calculated to express the likelihood of fertilization over the likelihood of non-fertilization, given normal LR + or abnormal test results LR-, as previously described by Collins (1987).

Correlations between individual test results and fertilization rates were evaluated by calculating Pearson's rank correlation coefficient.

Three categories were discerned in respect of patients' native and post-swim-up (MEUSC) scores:

Category 1: native $<5$ and post-swim-up $<8$, Category 2: native $<5$ or post-swim-up $<8$, Category 3: native $>5$ and post-swim-up $>8$.

The response of semen parameters to the swim-up technique were evaluated by comparing the parameters before and after the swim-up procedure using the paired $t$-test. Semen parameters were also evaluated according to their fertilization outcome by using the unpaired t-test.

The relationship between categories and fertilization outcome per oocyte, embryo transfer (ET) rate per patient, pregnancy rate per ET, and ongoing pregnancy rate/delivered baby rate per ET were examined using a Chi-square test in a $2 \times 2$ table, or Fisher's exact test in instances when the expected value in one of the cells was $\leq 5$. 
Figure 1. Receiver operating characteristic (ROC) curves of the best three parameters with respect to fertilization outcome.

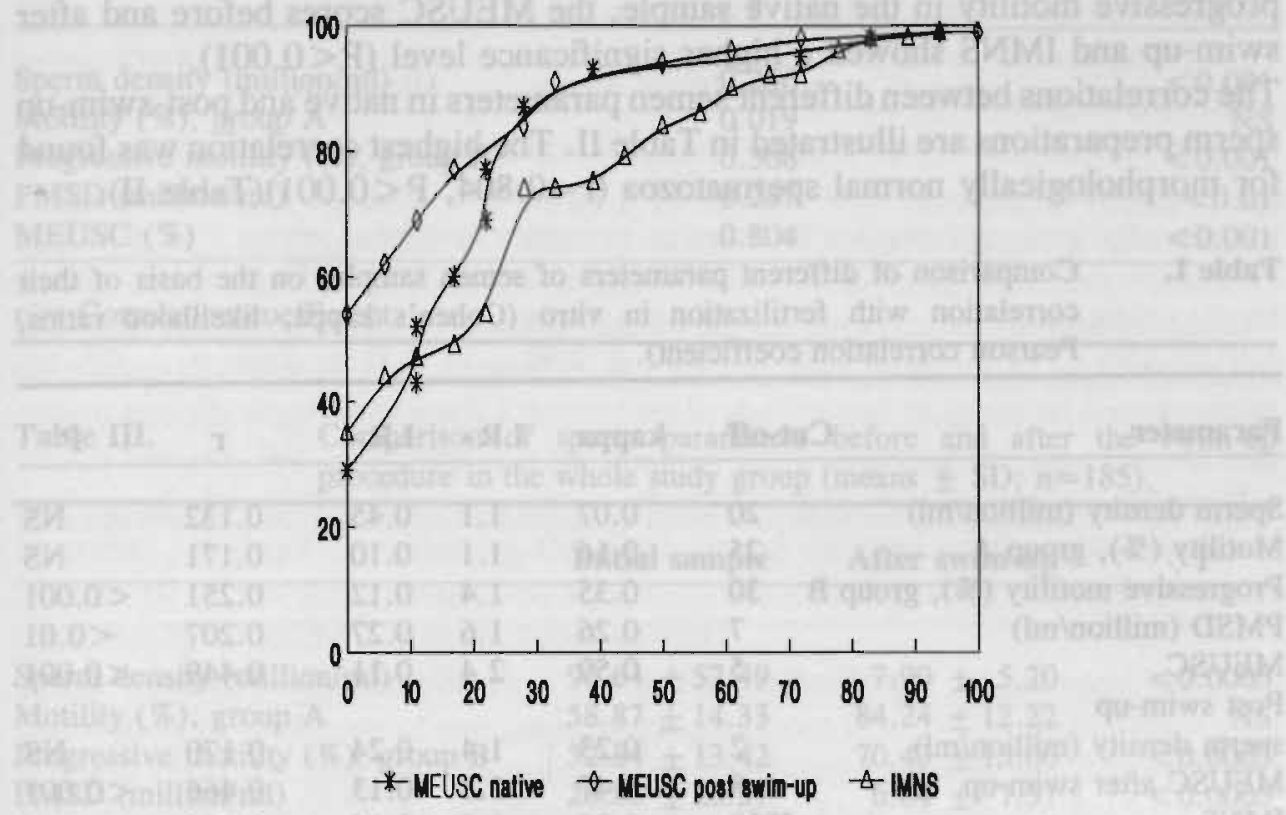

\section{Results}

For all the semen parameters ROC curves were constructed (Fig. 1). The ROC curve for an ideal diagnostic test reaches the upper left corner and encloses the largest area below and to the right of the curve. The two curves for the percentage of morphologically normal spermatozoa, before and after swim-up, enclosed a larger area than did the other parameters (not all shown in Fig. 1) and were almost identical. Although the enclosed area of IMNS was less then for the two curves just described, it was the third-best parameter in respect of the amount of area that was enclosed below and to the right of the curve.

When kappa values were calculated to find the clinically acceptable cut-off points, only both values for MEUSC were above the clinically acceptable 0.40 level (Table I). Likelihood ratios were also calculated: the likelihood of having a $>0 \%$ fertilization outcome (fertile) given normal test results LR + showed fair results for both of the sperm morphology scores, whereas all other parameters were inoperable. The likelihood of having a $>0 \%$ fertilization outcome (fertile) given abnormal test results LR- showed good results for motility and progressive motility of the native sample and for both sperm morphology scores, whereas all other parameters showed fair results (Table I).

After calculating the correlation coefficients, PMSD showed a significant 
correlation with the fertilization rate $(\mathrm{P}<0.01)$. However, the correlation between the fertilization rate and the percentage of spermatozoa exhibiting progressive motility in the native sample, the MEUSC scores before and after swim-up and IMNS showed a higher significance level $(\mathrm{P}<0.001)$.

The correlations between different semen parameters in native and post-swim-up sperm preparations are illustrated in Table II. The highest correlation was found for morphologically normal spermatozoa $(r=0.804, P<0.001)$ (Table II).

Table I. Comparison of different parameters of semen samples on the basis of their correlation with fertilization in vitro (Cohen's kappa, likelihood ratios, Pearson correlation coefficient).

\begin{tabular}{lrrrrrrr}
\hline Parameter & Cut-off & kappa & LR + & LR- & r & P \\
\hline Sperm density (million/ml) & 20 & 0.07 & 1.1 & 0.45 & 0.132 & NS \\
Motility (\%), group A & 25 & 0.16 & 1.1 & 0.10 & 0.171 & NS \\
Progressive motility (\%), group B & 30 & 0.35 & 1.4 & 0.12 & 0.251 & $<0.001$ \\
PMSD (million/ml) & 7 & 0.26 & 1.6 & 0.27 & 0.207 & $<0.01$ \\
MEUSC & 5 & 0.50 & 2.4 & 0.11 & 0.449 & $<0.001$ \\
Post swim-up & & & & & & \\
sperm density (million/ml) & 2 & 0.23 & 1.4 & 0.24 & 0.170 & NS \\
MEUSC after swim-up & 8 & 0.47 & 2.8 & 0.13 & 0.466 & $<0.001$ \\
IMNS & 5800 & 0.25 & 1.5 & 0.26 & 0.336 & $<0.001$ \\
\hline
\end{tabular}

$\mathrm{r}$ and $\mathrm{P}=$ Pearson's correlation coefficient; Kappa $=$ Cohen's kappa $(<0.4=$ poor agreement; $0.4-0.75=$ good agreement); $L R+=$ Likelihood of fertilization, given normal test result $(<2=$ inoperative; $2-5=$ fair; $5-10=$ good); $L R-=$ Likelihood of fertilization, given abnormal test result $(0.1-0.2=$ good; $0.2-0.5=$ fair; $>0.5=$ inoperative).

Significant differences were observed for all semen parameters except for group A sperm motility when the native and the post-swim-up sperm preparations were compared in the whole study group $(\mathrm{P}<0.0005)$ (Table III). These correlations were constant in the fertile group (Table IV) in which the fertilization rate was $>0 \%$. When the differences were evaluated in the infertile group the response to the swim-up survival was weaker than the for fertile group (Table V).

Semen parameters were evaluated with respect to the fertilization outcome (Table VI). Fertile and infertile groups were significantly. different from one another, apart from the percentage motility (group A). Progressive motility (group B) and sperm morphology scores showed the most significant differences between the infertile ( $0 \%$ fertilization rate) and fertile ( $>0 \%$ fertilization rate) groups $(\mathrm{P}<0.0005)$ whereas the difference in sperm density was the least significant $(\mathrm{P}<0.05)$. However, since the PMSD in the IVF medium was adjusted, the statistical significance between the groups was meaningful for sperm density, MEUSC, IMNS (Table VI). 
Table II. Correlation between pre- and post-swim-up sperm parameters.

\begin{tabular}{lcr}
\hline & r & P \\
\hline Sperm density (million/ml) & & $<0.001$ \\
Motility (\%), group A & 0.256 & NS \\
Progressive motility (\%), group B & 0.019 & $<0.001$ \\
PMSD (million/ml) & 0.368 & $<0.01$ \\
MEUSC (\%) & 0.287 & $<0.001$ \\
\hline & 0.804 & \\
r= Correlation coefficient. & & \\
\hline
\end{tabular}

Table III.

Comparison of sperm parameters before and after the swim-up procedure in the whole study group (means $\pm S D ; n=185$ ).

\begin{tabular}{lrrrr}
\hline & Initial sample & After swim-up & \multicolumn{1}{c}{ P } \\
\hline & & & \\
Sperm density (million/ml) & $90.64 \pm 57.49$ & $7.90 \pm 5.20$ & $<0.0005$ \\
Motility (\%), group A & $58.87 \pm 14.33$ & $84.24 \pm 12.22$ & NS \\
Progressive motility (\%), group B & $52.84 \pm 13.42$ & $70.43 \pm 15.00$ & $<0.0005$ \\
PMSD (million/ml) & $28.80 \pm 20.57$ & $6.84 \pm 7.37$ & $<0.0005$ \\
MEUSC (\%) & $9.22 \pm 4.02$ & $12.52 \pm 4.85$ & $<0.0005$ \\
\hline
\end{tabular}

Table IV. Comparison of sperm parameters before and after the swim-up procedure in the fertile (fertilization rate $>0 \%$ ) group (means $\pm S D ; n=167$ ).

\begin{tabular}{lrrrr}
\hline & Initial sample & After swim-up & P \\
\hline & & & \\
Sperm density (million/ml) & $91.45 \pm 57.47$ & $8.22 \pm 5.25$ & $<0.0005$ \\
Motility (\%), group A & $59.52 \pm 13.77$ & $84.61 \pm 12.16$ & NS \\
Progressive motility (\%), group B & $54.01 \pm 12.55$ & $71.92 \pm 14.14$ & $<0.0005$ \\
PMSD (million/ml) & $29.73 \pm 20.67$ & $7.33 \pm 7.59$ & $<0.0005$ \\
MEUSC (\%) & $9.67 \pm 3.86$ & $13.15 \pm 4.62$ & $<0.0005$ \\
\hline
\end{tabular}

Three categories were discerned to evaluate the combined effect of native and post-swim-up sperm morphology scores (Table VII). The fertilization rates were significantly different $(\mathrm{P}<0.000001)$. The embryo transfer rate per couple in categories 2 and 3 were significantly better than for category $1(P<0.005)$. There were no pregnancies in Category 1 and this was significantly different from both of the following categories $(P<0.02)$. There were no significant differences between categories 2 and 3 in respect to pregnancy rate or ongoing or delivered baby rate. 
Table V. Comparison of sperm parameters before and after the swim-up procedure in the inferile (fertilization rate $=0 \%$ ) group (means $\pm S D ; n=18$ ).

\begin{tabular}{lrrr}
\hline & Initial sample & After swim-up & P \\
\hline & & & \\
Sperm density (million/ml) & $83.11 \pm 58.80$ & $4.94 \pm 3.63$ & $<0.04$ \\
Motility (\%), group A & $52.78 \pm 18.09$ & $80.83 \pm 12.63$ & NS \\
Progressive motility (\%), group B & $41.94 \pm 16.46$ & $56.67 \pm 16.18$ & NS \\
PMSD (million/ml) & $22.25 \pm 17.94$ & $2.36 \pm 1.70$ & $<0.03$ \\
MEUSC (\%) & $5.00 \pm 2.93$ & $6.72 \pm 2.65$ & $<0.001$ \\
\hline
\end{tabular}

Table VI. Relationship between post-swim-up sperm parameters and fertilization outcome (means \pm SD).

\begin{tabular}{lrrrr}
\hline & Fertilization rate $=\mathbf{0 \%}$ & Fertilization rate $=\mathbf{0 \%}$ & P \\
\hline & & & & \\
Sperm density (million/ml) & $83.11 \pm 58.80$ & $4.94 \pm 3.63$ & $<0.04$ \\
Motility (\%), group A & $52.78 \pm 18.09$ & $80.83 \pm 12.63$ & NS \\
Progressive motility (\%), group B & $41.94 \pm 16.46$ & $56.67 \pm 16.18$ & NS \\
PMSD (million/ml) & $22.25 \pm 17.94$ & $2.36 \pm 1.70$ & $<0.03$ \\
MEUSC (\%) & $5.00 \pm 2.93$ & $6.72 \pm 2.65$ & $<0.001$ \\
\hline
\end{tabular}

Table VII. Comparison of different categories in respect to their IVF outcome.

\begin{tabular}{lrrrrr} 
Category* & n & $\begin{array}{c}\text { Fertilization } \\
\text { of oocytes }\end{array}$ & Embryo Transfer & $\begin{array}{c}\text { Pregnancy Ongoing/delivery } \\
\text { rate } \\
\text { rate }\end{array}$ \\
\hline 1 & 16 & $10 / 1377 \%^{\mathrm{a}}$ & $5 / 1631 \%^{\mathrm{d}}$ & $0 \%^{\mathrm{f}}$ & $0 \%^{\mathrm{f}}$ \\
2 & 17 & $94 / 18551 \%^{\mathrm{b}}$ & $14 / 1782 \%^{\mathrm{c}}$ & $29 \%^{\mathrm{g}}$ & $29 \%^{\mathrm{g}}$ \\
3 & 152 & $1103 / 158470 \%^{\mathrm{c}}$ & $145 / 15295 \%^{\mathrm{c}}$ & $23 \%^{\mathrm{g}}$ & $20 \%^{\mathrm{g}}$ \\
\hline
\end{tabular}

Different superscripts in the same column are significantly different from one another: $a, b, c$ Chi-Square test $P<0.000001$; d,e Fisher's exact test $P<0.005 ;$ f,g Fisher's exact test $P<0.02$;

"MEUSC categorized as 1 : native $<5$ and post-swim-up $<8$; 2 : native $<5$ or post-swim-up $<8$; 3 : native $>5$ and post-swim-up $>8$. 


\section{Discussion}

The potential usefulness of IVF results for studying the value of sperm parameters for predicting fertilization outcome has been shown previously (Enginsu et al., 1992b; Kruger et al., 1988; Liu et al., 1990; Comhaire et al., 1988). It is clear at the moment that the percentage of spermatozoa with normal morphology may play an important role in this context. The value of using swim-up as a sperm selection criterion in terms of morphology has been shown earlier (Scott et al., 1989; Daya et al., 1989).

It is an established fact that after the swim-up procedure the sperm density decreases drastically (Mc Dowel et al., 1985). The problem of evaluating the sperm morphology after such a procedure is the limited number of spermatozoa which remain. We overcame this problem by centrifugating the supernatant that was used for IVF. The negative effects of this procedure is debatable. However, without considering the final sperm density of the post swim-up sample all of the supernatant was subjected to centrifugation. This gave us the opportunity to study a homogeneous group.

In our center 50000 progressively motile spermatozoa per $\mathrm{ml}$ are used for IVF. Since the IVF outcome of our patients ranged from 0 to $100 \%$ fertilization rate it might be concluded that the number of progressively motile spermatozoa has very limited effect on the fertilization outcome. Thus the sperm density after swim-up preparation and the percentage with normal morphology are the other two parameters to be evaluated. The results showed that the highest predictive value for IVF outcome was the number of morphologically normal spermatozoa, both in the native and post swim-up samples. Moreover, only these two parameters gave clinically acceptable cut-off points ( $5 \%$ and $8 \%$ respectively). However, correlation between the fertilization outcome and the percentage and density of progressively motile spermatozoa in the native semen sample shows that morphology, progressive motility and progressive motile sperm density play a role in establishing IVF outcome.

The highest correlation between the pre- and post swim-up results show that sperm morphology scores were the most powerful parameter for predicting post swim-up survival ( $r=0.804$, Table II).

The significant improvement in spermatozoa exhibiting progressive motility and normal morphology after the swim-up procedure in the whole group was clear (Table III). However, the responses of the fertile and infertile groups were different. The improvement in the percentage of spermatozoa with progressive motility was not significant in the infertile group whereas it was significantly different in the fertile group $(\mathrm{P}<0.0005)$. The improvement in the percentage of morphologically normal spermatozoa in both groups was again different in infertile and fertile groups ( $\mathrm{P}<0.001$ and $\mathrm{P}<0.0005$ respectively). Jeulin et al. (1986) also studied the effect of sperm selection procedures and showed the degree of improvement did not differ significantly between groups with low and 
high IVF success. However, according to others (Oehninger et al., 1990; Scott et al., 1989) the percentage of morphologically normal spermatozoa improves more in patients in which the IVF results are poor than in those with a good IVF outcome. In our study, the response to the swim-up was better in the fertile group, and the percentage of progressively motile spermatozoa improved significantly in the fertile group after swim-up, whereas there was no significant improvement in the infertile group (Tables IV and V). The only parameter which improved significantly in both fertile and infertile groups was the sperm morphology score. However, the improvement in this parameter by swim-up was significantly better in the fertile group $(3.48 \pm 2.91)$ than in the infertile group $(1.72 \pm 2.16)(\mathrm{P}<0.02)$ (Tables IV and V).

When the post-swim-up portions were evaluated individually the parameters remaining were sperm density, the percentage of spermatozoa with normal morphology and the number of morphologically normal spermatozoa used for IVF, since the progressive motile sperm density was adjusted to the same level in the IVF medium. When the post-swim-up sperm density was compared to the number of morphologically normal spermatozoa used for IVF, it appeared that both of the parameters gave kappa values that were below the acceptable limits ( 0.23 and 0.25 respectively). The likelihood ratios for both normal and abnormal test results were similar to each other (inoperable for normal test results and fair agreement for abnormal test results). The only parameter which was left was the sperm morphology score (MEUSC) in the post-swim-up sample. However the only new information about this parameter is the new cutoff point. The high correlation between the native and post swim-up samples in terms of percentage spermatozoa with normal morphology, suggests that even before the swim-up preparation the outcome of IVF is highly predictable.

On the other hand although IMNS showed a mild correlation with the fertilization outcome $(0.336, \mathrm{P}<0.001)$ though this did not give clinically acceptable cut-off points, but the fact that this correlation was not observed for the postswim-up sperm density shows that spermatozoa used for IVF plays a role in determining IVF outcome. It is also clear that when the fertile and infertile groups were compared in terms of their post-swim-up density, MEUSC and IMNS, the latter was the second-best criterion $(\mathrm{P}<0.001)$ after the morphology scores $(\mathrm{P}<0.0005)$; indeed the total sperm density after swim-up affects the predictive value of this parameter.

It is clear from the results that fertilization can occur in the group of patients in which both the native and the post-swim-up samples showed poor results (category 1). However, although fertilization occurred, there were no pregnancies in this group. The higher pregnancy rates in category 2 when compared to category 3 can be attributed to the limited number of couples in category 2 , though the difference was not significant.

A review on male infertility by Yates and de Kretser (1987) stresses the fact that IVF seems to be one of the most effective treatments for male-factor 
infertility. It is important to have a patient selection criteria before the IVF (Enginsu et al., 1992b). This will also allow tailoring of the sperm density used in vitro to improve IVF outcome, especially when a male factor is involved. It is concluded that (1) Sperm density in the IVF medium is not correlated with the fertilization outcome, whereas the morphologically normal sperm density shows good correlation with the fertilization outcome. Therefore morphology of the spermatozoa is of pivotal importance in determining fertilization in vitro. However, it is not possible to give a reliable cut-off point for this parameter. Since it is determined by both the final sperm density and their morphology scores, it is likely that sperm density has a negative effect. This makes the number of morphologically normal spermatozoa used for IVF a less reliable criterion. (2) Human sperm morphology evaluated by strict criteria is an important predictor of fertilization in vitro, both in the IVF medium and in the native sample. (3) Clinically reliable cut-off points are $5 \%$ and $8 \%$ for native and post-swim-up morphology scores respectively. These can be used as patient selection criteria for in vitro fertilization and as possible indicators of male subfertility.

\section{References}

Collins JA (1987): Diagnostic assessment of the infertile male partner. Curr Prob Obs Gyn Inf, 10: 175-224.

Comhaire FH, Vermeulen L, Hinting A, and Schoonjans F (1988): Accuracy of sperm characteristics in predicting the in vitro fertilizing capacity of semen. J IVF ET, 5: 326331.

Daya S, Gunby J, Kohut J (1989): Semen predictors of in vitro fertilization and embryo cleavage. Am J Obs Gyn, 161: 1284-1289.

Diamond MP, Rogers BJ, Vaughn WK, Wentz AC (1985): Effect of the number of inseminating sperm and the follicular stimulation protocol on in vitro fertilization of human oocytes in male factor and non-male factor couples. Fertil Steril, 44: 499-503.

Dumoulin JCM, Bras M, Land JA, Pieters MHEC, Enginsu ME, Evers JLH (1991): A high number of inseminating sperm in human in vitro fertilization is detrimental for the subsequent embryonic development in vitro. Hum Rep, 6(Suppl): 191.

Dumoulin JCM, Bras M. Land JA, Pieters MHEC. Enginsu ME, Geraedts JPM, Evers JLH (1992): Effect of the number of inseminating sperm in human in vitro fertilization on the subsequent embryonic development in vitro. Hum Rep, 7: 1010-1013.

Enginsu ME, Dumoulin JCM, Pieters MHEC, Bras M, Evers JLH, Geraedts JPM (1991): Evaluation of human sperm morphology using strict criteria after Diff-Quik staining: Correlation of morphology with fertilization in vitro. Hum Rep, 6: 854-858.

Enginsu ME, Dumoulin JCM. Pieters MHEC, Bergers M, Evers JLH, Geraedis JPM (1992a): Comparison between the hypoosmotic swelling test and morphology evaluation using strict criteria in predicting in vitro fertilization (IVF). J Ass Rep Gen, 9: 259-264

Enginsu ME, Dumoulin JCM, Land JA, Evers JLH, Geraedts JPM (1992b): Male factor ass determinant of in vitro fertilization outcome. Hum Rep, 7: 1136-1140.

Fleis JL (1981): Statistical Methods for Rates and Proportions. Toronto. John Wiley \& Sons $1981,56$.

Francavilla F, Romano R, Santucci R, Poccia G (1990): Effect of sperm morphology and motile sperm count on outcome of intrauterine insemination in oligozoospermia and/or 
asthenozoospermia. Fertil Steril, 53: 892-7.

Glazener CMA, Kelly NJ, Weir MJA, David JSE, Cornes JS, Hull MGR (1987): The diagnosis of male infertility-prospective time specific study of conception rates related to seminal analysis and post-coital sperm-mucus penetration and survival in otherwise unexplained infertility. Hum Rep, 2: 665-671.

Jeulin C, Feneux D, Serres C, Jouannet P, Guillet-Rosso F, Belaisch-Allart J, Frydman R, Testart J (1986): Sperm factors related to failure of human in-vitro fertilization. J Rep Fert, 76: 735-44.

Kruger TF, Swanson RJ, Hamilton M, Simmons KF, Acosta AA, Matta JF, Oehniger S, Morshedi M (1988): Abnormal sperm morphology and other semen parameters related to the outcome of the hamster oocyte human sperm penetration assay. Int J Androl, 11: 107-13.

Kruger TF, Menkveld R, Stander FSH, Lombard CJ, Merwe JP (1986): Sperm morphological features as a prognostic factor in in vitro fertilization. Fertil Steril, 46: 1118-1123

Liu DY, Baker, HW (1990): Relationships between human sperm acrosin, acrosomes, morphology and fertilization in vitro. Hum Rep, 5: 298-303.

Mahadevan M, Baker G (1984): Assessment and preparation of semen for in vitro fertilization; in Wood C, Trounson A (ed): Clinical In Vitro Fertilization. Berlin, SpringerVerlag, 83-97.

McDowel JS, Veeck LL, Jones HW (1985): Analysis of human spermatozoa before and after processing for in vitro fertilization. J IVF ET, 2: 23-26

Metz C (1978): Basic principles of ROC analysis. Sem Nuc Med, 8: 283-298.

Oehninger S, Acosta R, Morshedi M, Philput C, Swanson RJ, Acosta AA (1990): Relationship between morphology and motion characteristics of human spermatozoa in semen and in the swim-up sperm fractions. J Androl, 11: 446-52.

Quinn P, Warnes GM, Kerin JF, Kirby C (1984): Culture factors in relation to the success of human in vitro fertilization and embryo transfer. Fertility and Sterility, 4: 202-209.

Scott RT Jr, Oehninger SC, Menkveld R, Veeck LL, Acosta AA (1989): Critical assessment of sperm morphology before and after double wash swim-up preparation for in vitro fertilization. Arch Androl, 23: 125-9.

WHO, Laboratory Manual for the Examination of Human Semen and Sperm-Cervical Mucus Interactions (1987): Cambridge University Press, Cambridge 2nd edn, pp.9-11.

Wolf DP, Byrd W, Dandekar P, Quigley MM (1984): Sperm concentration and the fertilization of human eggs in vitro. Biol Rep, 31: 837-848.

Yates CA, and Kretser DM (1987): Male-factor infertility and in vitro fertilization. J IVF ET, 4: 141-147. 


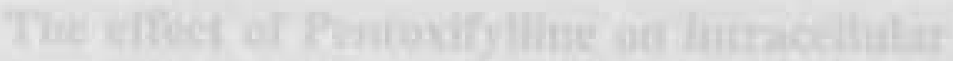

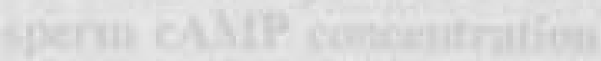

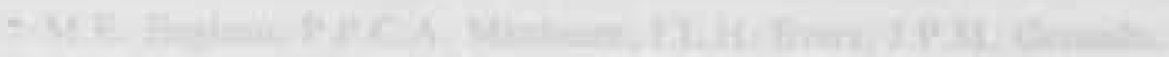

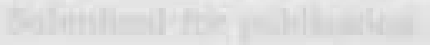

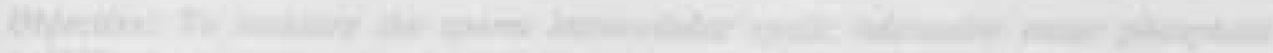

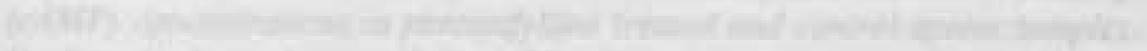

H.

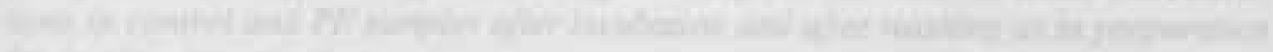

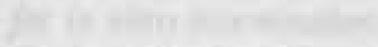

and

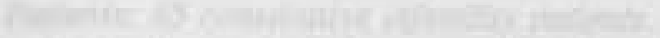

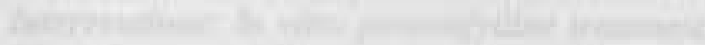

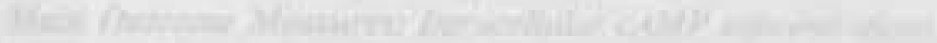

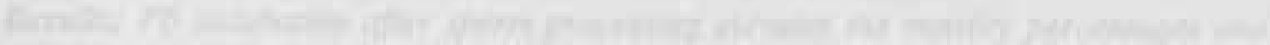

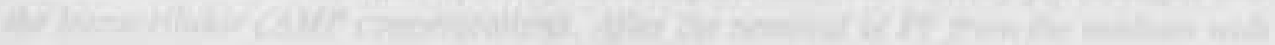
a

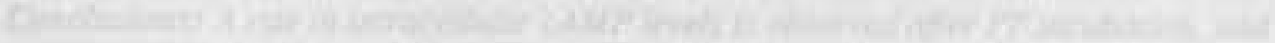

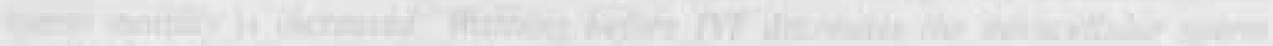

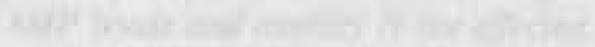

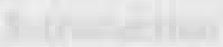

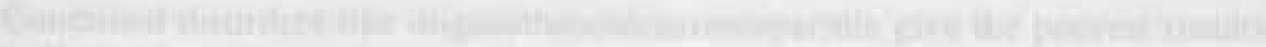

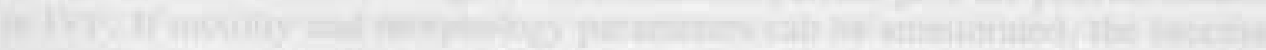

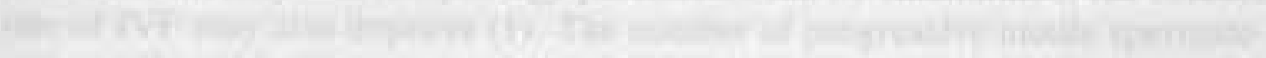

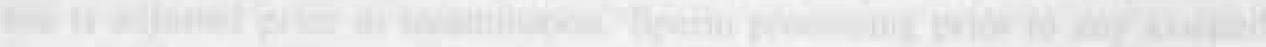

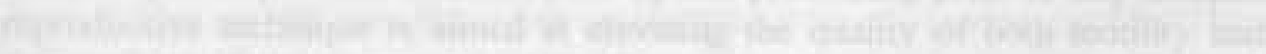

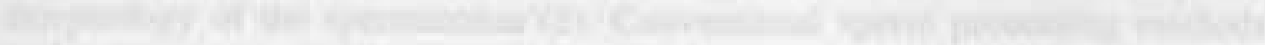

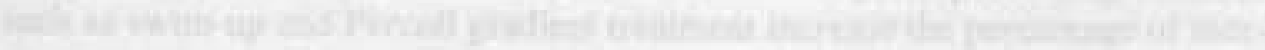

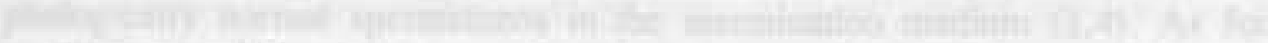

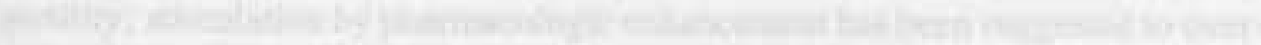

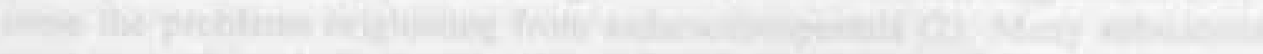

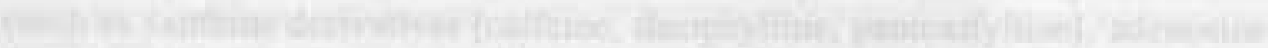

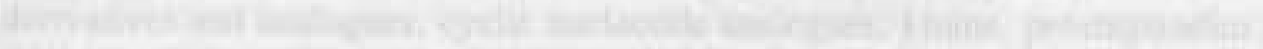

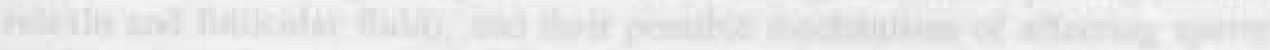

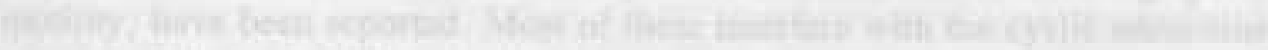

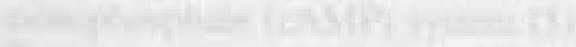

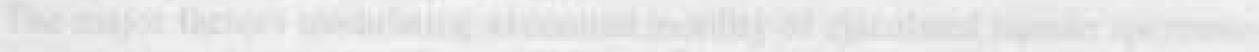

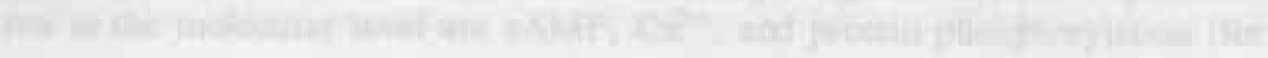




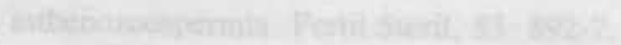

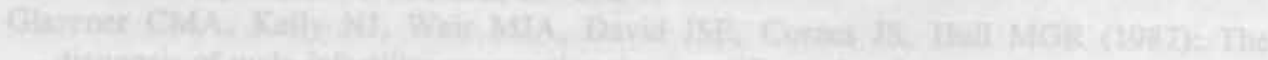

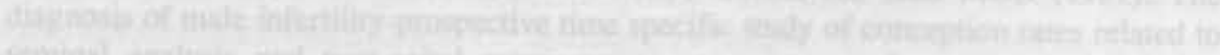

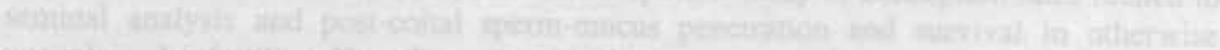

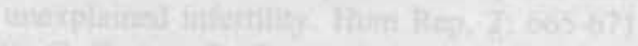

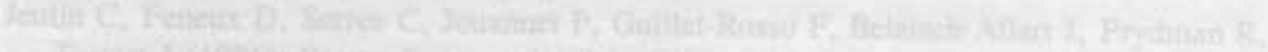

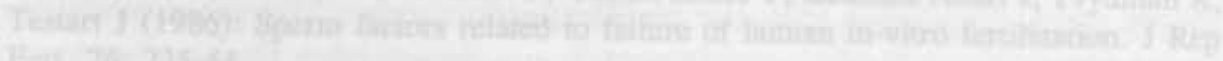

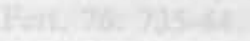

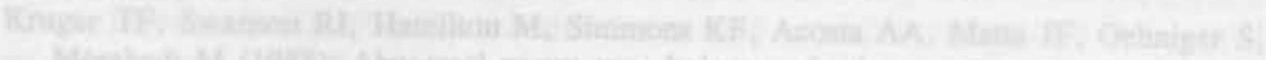

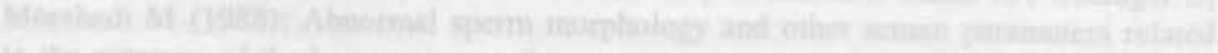

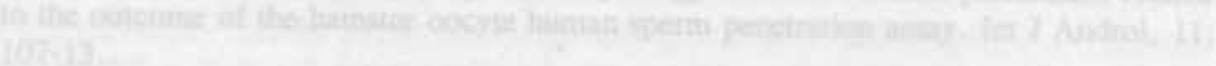

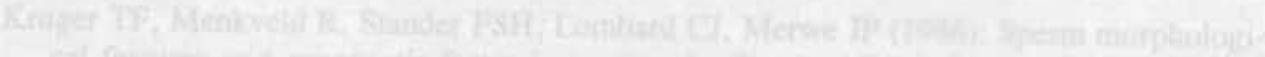

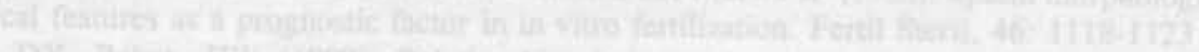

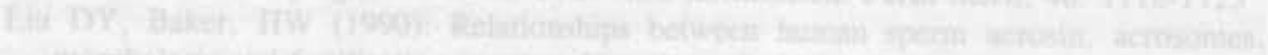

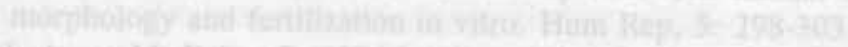

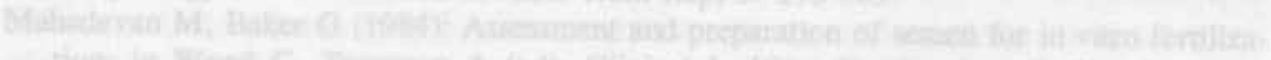

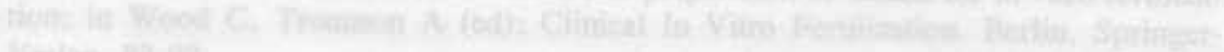

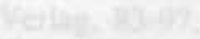

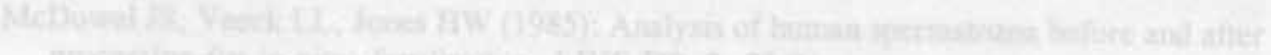

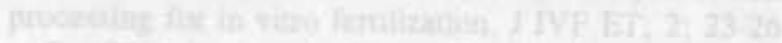

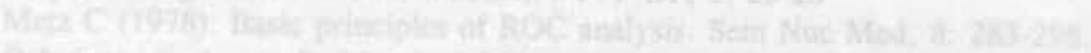

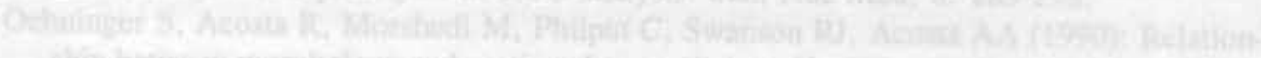

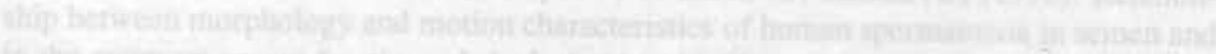

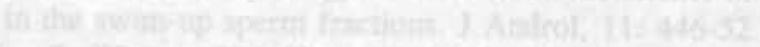

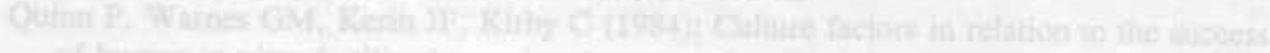

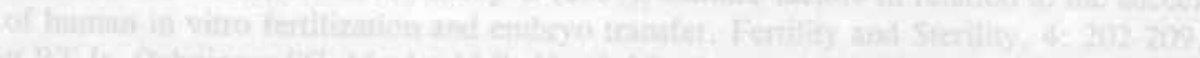

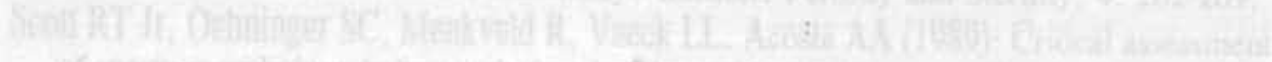

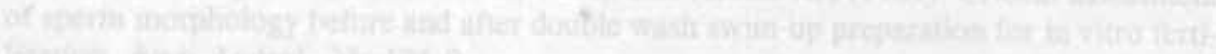

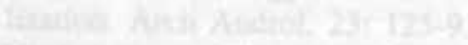

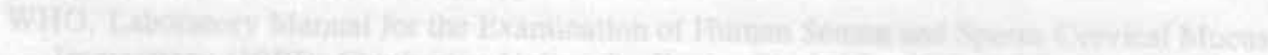

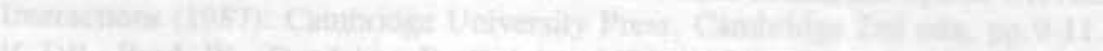

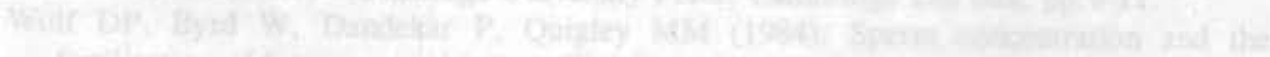

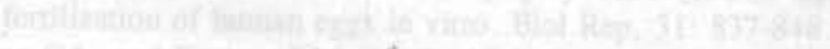

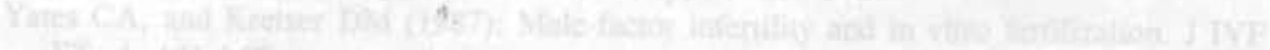

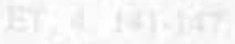




\title{
The effect of Pentoxifylline on intracellular sperm cAMP concentration
}

\author{
M.E. Enginsu, P.P.C.A. Menheere, J.L.H. Evers, J.P.M. Geraedts. \\ Submitted for publication
}

Objective: To measure the sperm intracellular cyclic adenosine mono phosphate (cAMP) concentrations in pentoxifylline treated and control sperm samples.

Design: Analysis of pentoxifylline (PF) effects on sperm intracellular cAMP concentrations in control and $P F$ samples after incubation and after washing as in preparation for in vitro insemination.

Setting: Academic hospital, fertility laboratory.

Patients: 65 consecutive infertility patients.

Interventions: In vitro pentoxifylline treatment.

Main Outcome Measures: Intracellular cAMP concentrations.

Results: $P F$ incubation after sperm processing elevates the motility percentages and the intracellular $C A M P$ concentrations. After the removal of $P F$ from the medium with washing steps, intracellular CAMP concentrations were decreased.

Conclusions: A rise in intracellular cAMP levels is observed after $P F$ incubation, and sperm motility is increased. Washing before IVF decreases the intracellular sperm cAMP levels and motility is not effected.

\section{Introduction}

Combined disorders like oligoasthenoteratozoospermia give the poorest results in IVF. If motility and morphology parameters can be ameliorated, the success rate of IVF may also improve (1). The number of progressive motile spermatozoa is adjusted prior to insemination. Sperm processing prior to any assisted reproductive technique is aimed at elevating the quality of both motility and morphology of the spermatozoa (2). Conventional sperm processing methods such as swim-up and Percoll gradient treatment increase the percentage of morphologically normal spermatozoa in the insemination medium $(3,4)$. As for motility, stimulation by pharmacologic enhancement has been suggested to overcome the problems originating from asthenozoospermia (2). Many substances (such as xanthine derivatives [caffeine, theophylline, pentoxifylline], adenosine derivatives and analogues, cyclic nucleotide analogues, kinins, prostaglandins, relaxin and follicular fluid), and their possible mechanisms of affecting sperm motility, have been reported. Most of these interfere with the cyclic adenosine monophosphate (cAMP) system (5).

The major factors modulating axonemal motility of ejaculated human spermatozoa at the molecular level are cAMP, $\mathrm{Ca}^{2+}$, and protein phosphorylation (for 
review see 6). The major stimulatory pathways are: 1 - stimulation of production of cAMP by adenylate cyclase, 2- reduction of metabolization of cAMP by inhibition of cyclic nucleotide phosphodiesterases, and 3- supplementation of intracellular cAMP levels by polar analogues capable of diffusing through cell membranes (5). cAMP also is the only cyclic nucleotide shown to have a beneficial role on human sperm activity (7).

Methylxanthine derivatives like caffeine and theophylline increase intracellular cAMP levels by inhibiting phosphodiesterase activity, which is responsible for the further metabolization of cAMP in human spermatozoa $(8,9)$.

Pentoxifylline (PF), another methylxanthine derivative, has been widely used as a motility stimulant both in vivo (10-13) and in vitro (14-16). However, conflicting results have been reported on the stimulatory effect of pentoxifylline in in vitro studies (14-16).

The aim of this study was to evaluate the effect of pentoxifylline stimulation on sperm, prepared as for IVF, and to validate the laboratory assay, used to measure the intracellular cAMP levels in human spermatozoa.

\section{Materials and Methods}

Two experiments were performed (see Figure 1). In Experiment 1 the effect of pentoxifylline on sperm intracellular cAMP was measured after preparing the semen as for IVF. In Experiment 2 sperm intracellular cAMP concentrations were measured immediately after PF incubation, and after washing. Motility parameters after incubation were also evaluated in both experiments.

Semen samples from 65 consecutive infertility patients were included in this study. Forty-one samples were used for Experiment 1 and 24 for Experiment 2 (see Figure 1). Semen analysis was performed prior to sperm processing and after incubation. In neither experiment was a selection made in respect of the patient's semen analysis. In Experiment 1, 54\%, and in Experiment 2, 67\% of the samples were normozoospermic. The sperm sample was divided into two equal aliquots so as to be used as its own control (Figure 1).

Conventional methods were used for semen analysis. Sperm density and motility parameters were assessed in a Makler counting chamber, as published previously (1). Briefly, two classes of sperm motility were recognized: slow, sluggish movement or movement of the spermatozoa hampered by an obvious, clearly visible morphological defect (\% motility) and rapid and linear progressive movement (\% progressive motility). The total number of the progressive motile spermatozoa was defined as progressive motile sperm density (PMSD).

\section{Experiment 1}

A suspension of motile spermatozoa was prepared with the use of a two layer Percoll gradient ( $85 \%$ and $70 \%$ ). After Percoll washing the pellet was diluted 
with $3 \mathrm{ml}$ of human tubal fluid (HTF) (17) supplemented with $10 \%$ human serum protein solution (GPO)(Central Laboratory of the Blood Transfusion Service, Amsterdam, the Netherlands) and divided into two equal parts as control and treatment sample. After two washes with HTF-GPO (to wash Percoll out), the final pellets were diluted with either HTF-GPO alone (control group) or with HTF-GPO containing $1 \mathrm{mg} / \mathrm{ml}(=3.6 \mathrm{mM}$ ) pentoxifylline (Sigma chemie, Axel, NL)(treatment group). After $1 \mathrm{~h}$ incubation in $5 \% \mathrm{CO}_{2}$, sperm density, percentage motility and PMSD were evaluated in both samples. To wash out pentoxifylline, both the control and the treated samples were diluted with $3 \mathrm{ml}$ HTF-GPO and centrifugated twice. The supernatants were discarded. The final pellet was diluted with $0.5 \mathrm{ml} \mathrm{HTF-GPO}$ and used or the cAMP measurement.

\section{Experiment 2}

The Percoll and incubation steps were identical to those in Experiment 1. After the 1 hour incubation in $5 \% \mathrm{CO}_{2}$ both the control and the pentoxifylline samples were divided into two equal portions. One portion from each specimen was used for CAMP measurement immediately following the incubation whereas the other portion was subjected to the further washing procedure as described in Experiment 1.

\section{Extraction}

Prior to the cAMP measurement, cAMP was extracted from the cells with a protocol modified from Aitken et al.(9). Final solutions from all experiments were centrifugated for 10 minutes at $4^{\circ} \mathrm{C}(1610 \mathrm{~g})$. The supernatant was discarded from the tubes and $3 \mathrm{ml}$ ice cold ethanol supplemented with $20 \mathrm{mmol}$ $\mathrm{HCl}$ were added to the pellet. The tubes were placed in the ultrasound bath for 30 minutes to destroy the plasma membrane of spermatozoa and enable the intracellular cAMP to escape from the cell. After slow rotation at $4^{\circ} \mathrm{C}$ for 1 hour $(20 \mathrm{rpm})$, the samples were centrifugated for 10 minutes at $4^{\circ} \mathrm{C}$. The supernatants were transferred into other tubes and dried in nitrogen.

\section{Measurement of intracellular cAMP}

Intracellular sperm cAMP concentrations, from the extracted samples were estimated using a commercial cAMP kit (INCSTAR, Stillwater, Minnesota, USA). The assay is of an equilibrium radioimmunoassay. Samples were mixed with the first antibody (rabbit anticAMP antiserum diluted in BSA-sodium acetate buffer) and tracer (succinyl cAMP tyrosine methyl ester labelled with ${ }^{125} \mathrm{I}$ ) and incubated overnight at $4^{\circ} \mathrm{C}$. Next day a pre-precipitated second antibody (goat anti-rabbit antiserum with polyethylene glycol (PEG) diluted in BSA-borate buffer) complex is added to separate the bound fraction from the 
Figure 1. Schematic explanation of Experiment 1 and Experiment 2.

\section{EXPERIMENT 1}

Sperm processing with 2 layer Percoll gradients $(70 \%-85 \%)$ and $2 \mathrm{x}$ centrifugation to wash Percoll out

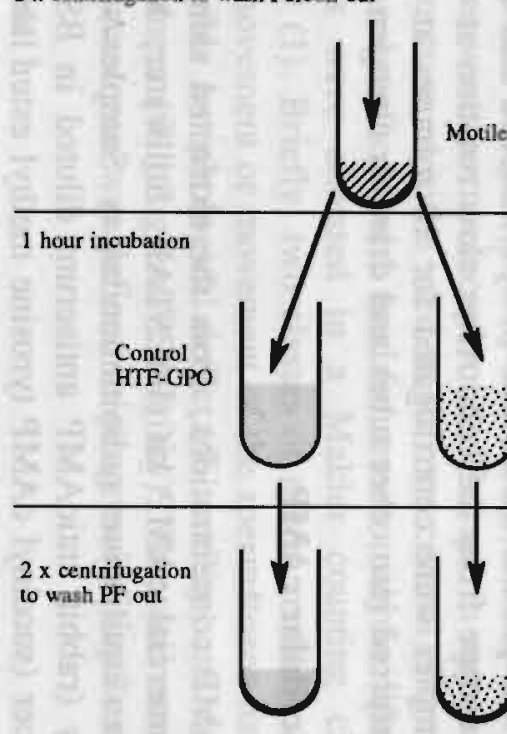

cAMP measured in these pellets

\section{EXPERIMENT 2}

Sperm processing with 2 layer Percoll gradients $(70 \%-85 \%)$ and $2 x$ centrifugation to wash Percoll cut

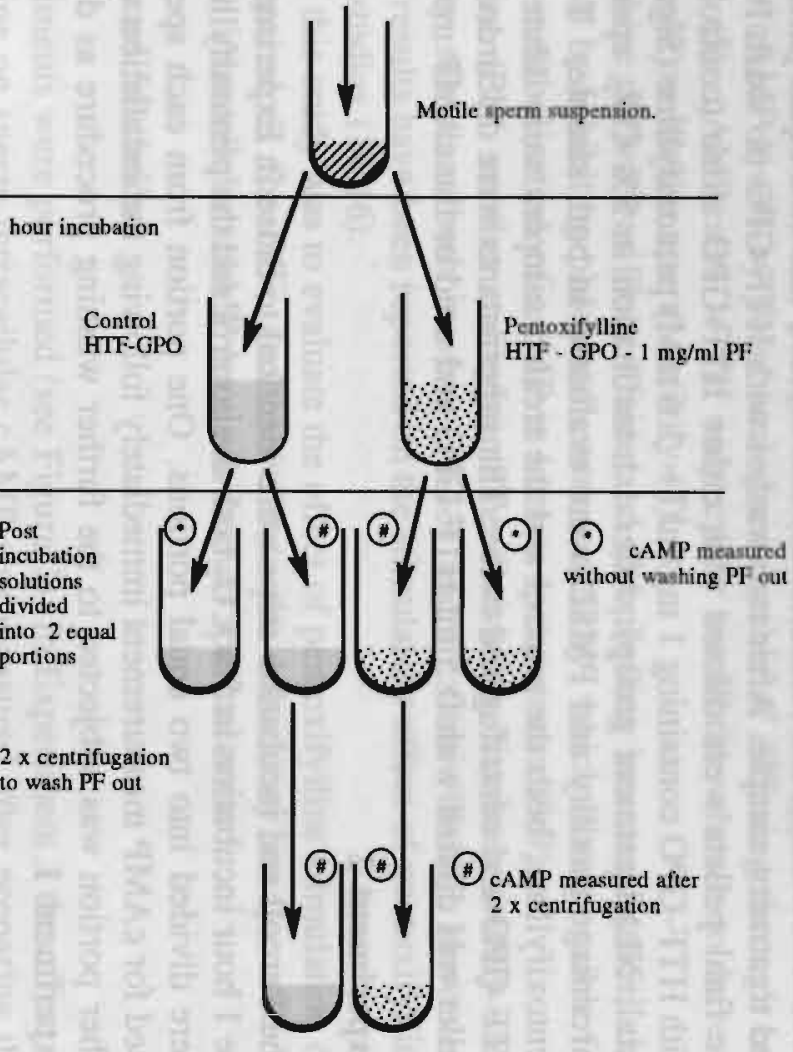


free fraction of cAMP. Finally the bound fraction is measured on a multihead gamma counter (Wizzard, Wallac Ltd, Turku, Finland). Data reduction has been done with the $\operatorname{logit}-\log 4$ parameter model. Cross reactivity with AMP, ADP, ATP and cGMP were less than $0.01 \%$.

\section{Statistics}

Different semen parameters in the native, control and pentoxifylline treated groups were compared with paired Student's t-test. Intracellular cAMP measurements were evaluated also with paired Student's t-test. In Experiment 2 the data from 4 groups were evaluated after arcsin transformation and analyzed using an analysis of variance (ANOVA) for randomized blocks experiments (18).

\section{Results}

In Experiment 1 the sperm densities in the control group and PF group were not significantly different. The same was found in Experiment 2 (Tables I and II). In both experiments the motility, progressive motility and PMSD were significantly higher in the pentoxifylline group than in the control group (Tables I and II).

Table I. Semen parameters and sperm intracellular cAMP concentrations for Experiment $1(n=41)$. The values are given as Mean \pm S.E.M.

\begin{tabular}{lrrr}
\hline Experiment 1 & NATIVE & CONTROL & PENTOXI \\
\cline { 2 - 4 } Sperm density (million/ml) & $58.8 \pm 9.1^{\mathrm{a}}$ & $19.2 \pm 2.8^{\mathrm{b}}$ & $21.4 \pm 3.8^{\mathrm{b}}$ \\
\% Motility & $45.6 \pm 2.7^{\mathrm{c}}$ & $53.0^{\mathrm{f}} \pm 3.2^{\mathrm{b}}$ & $68.8 \pm 2.5^{\mathrm{c}}$ \\
\% Progressive motility & $21.4 \pm 2.3^{\mathrm{f}}$ & $27.5 \pm 2.8^{\mathrm{g}}$ & $40.7 \pm 2.7^{\mathrm{h}}$ \\
PMSD (million/ml) & $17.6 \pm 3.4^{\mathrm{i}}$ & $4.9 \pm 1.9^{\mathrm{j}}$ & $11.0 \pm 2.3^{\mathrm{k}}$ \\
cAMP After incubation \& wash & & $73.6 \pm 7.3$ & $82.2 \pm 9.0$ \\
(femtomol cAMP per sperm) & - & &
\end{tabular}

${ }^{a-k}$ Within the lines, different superscripts show significant difference $(p<0.05)$.

In Experiment 1, intracellular cAMP concentrations per spermatozoon were not significantly different between the control group and pentoxifylline group $(p=0.13)($ Table II).

In Experiment 2 intracellular cAMP concentrations per spermatozoon of the PF treated group showed a significant increase on the samples where PF was not washed out $(\mathrm{p}<0.0005)$ (Table III). When the intracellular cAMP concentrations were measured on the other half of the samples after the washing steps, the increase was lost $(p=0.18)$. 
Table II. Semen parameters for Experiment $2(n=24)$. The values are given as Mean \pm S.E.M.

\begin{tabular}{lrrr} 
Experiment 2 & NATIVE & CONTROL & PENTOXI \\
\hline Sperm density (million/ml) & $68.9 \pm 10.7^{\mathrm{a}}$ & $25.8 \pm 4.8^{\mathrm{b}}$ & $26.5 \pm 5.0^{\mathrm{b}}$ \\
\% Motility & $47.1 \pm 2.8^{\mathrm{c}}$ & $58.5 \pm 3.1^{\mathrm{d}}$ & $67.0 \pm 3.0^{\mathrm{c}}$ \\
\% Progressive motility & $24.8 \pm 2.4^{\mathrm{f}}$ & $34.5 \pm 4.7^{\mathrm{g}}$ & $40.8 \pm 3.4^{\mathrm{h}}$ \\
PMSD (million/ml) & $19.7 \pm 4.7^{\mathrm{i}}$ & $6.8 \pm 1.9^{\mathrm{j}}$ & $11.7 \pm 3.0^{\mathrm{k}}$ \\
\hline
\end{tabular}

${ }^{a-k}$ Within the lines, different superscripts show significant difference $(p<0.05)$.

Table III. Intracellular cAMP concentrations for Experiment $2(n=24)$. The values are given as Mean \pm S.E.M.

Experiment 2

CONTROL

PENTOXI

cAMP After incubation

(femtomol cAMP per sperm)

$81.6 \pm 8.4$

$150.3 \pm 13.5^{*}$

cAMP After incubation \& wash

(femtomol cAMP per sperm)

$78.1 \pm 7.7$

$91.6 \pm 13.2$

* Significantly different from all other 3 groups (ANOVA, Tukey, p $<0.0005$ ).

The absence of a cross reaction of pentoxifylline with the antibody versus cAMP was checked by using a sample of containing distilled water containing $3.6 \mathrm{mM}$ PF. No cross reaction was detected.

\section{Discussion}

The stimulation of motility by pentoxifylline has been well documented (10-13). The results obtained from both experiments are in agreement with these previous findings regarding the elevation of percentage progressive motility and progressive motile sperm density in the pentoxifylline treated group.

With the help of CASA systems, it has recently been shown that pentoxifylline increases the curvilinear velocity, path velocity, lateral head displacement, beat cross frequency, and sperm hyper activation of individual spermatozoa $(19,20)$. Since in our study all motility parameters were assessed visually in a Makler counting chamber and no CASA system was at our disposal, we cannot comment on the velocity of the spermatozoa.

The absence of a significant difference in the intracellular cAMP measurements between the control and PF treated group in Experiment 1 shows that increased cAMP levels do not exist in the post processing samples, prepared as in the IVF 
laboratory routine.

The results obtained from Experiment 2 indicate that incubation with pentoxifylline can indeed increase intracellular cAMP concentrations immediately after incubation, as suggested previously for other methylxanthine derivatives $(8,9)$ and also for pentoxifylline (21). When pentoxifylline was removed from the samples this rise in intracellular cAMP was eliminated again.

It is known that capacitation and acrosome reaction are prerequisites for fertilization. Capacitation is a reversible process, whereas acrosome reaction is irreversible. Recent studies suggest that cAMP is not only responsible for capacitation and hyperactivation $(22,23)$ but for the acrosome reaction as well $(24,25)$.

Pentoxifylline maintains its stimulatory effect on the spermatozoa up to 4 hours after its removal, either in frozen thawed samples (26) or in fresh samples, treated as for IVF $(19,27)$. Experimental evidence on human spermatozoa supports the role of the cAMP second-messenger system in acrosome reaction induction (24). The incubation of spermatozoa with pentoxifylline is responsible for the sensitization of spermatozoa to the physiological acrosome reaction which takes place after binding of the spermatozoon to the zona pellucida (25). In contrast to PF, caffeine activates the spontaneous acrosome reaction, which is disadvantageous for the sperm fertilizing ability if it takes place during incubation and precedes zona binding (25). When these results are combined with our observations, it can be concluded that the rise in intracellular cAMP levels has an irreversible effect on spermatozoa.

The literature is inconsistent about the effect of pentoxifylline in IVF procedures. It was reported that in patients with severe male infertility (15), or with a defect on the acrosome reaction (28) pentoxifylline treatment showed a beneficial effect. However, when pentoxifylline treatment was applied to patients with a previous IVF failure (without any male factor detected) there was no therapeutic advantage (16).

It is undesirable to bring the oocyte into contact with pentoxifylline containing medium, since the absence of toxic effects of pentoxifylline on early embryo development has not yet been well established. Moreover, elevated cAMP levels causes the inhibition of germinal vesical breakdown and further oocyte maturation, which requires the removal of PF from the treated samples (29). However, from our results and from previous findings $(19,27)$ it might be assumed that once the cAMP levels have become elevated, further washing does not affect the hyperactivation and sensitization of the spermatozoa to the spontaneous acrosome reaction, although the cAMP levels may decrease. Therefore, pentoxifylline can be used to enhance the progressive motility. Removing pentoxifylline after incubation will not disturb the stimulatory effect.

\section{References}

1. Enginsu ME, Dumoulin JCM, Land JA, Evers JLH, Geraedts JPM (1992): Male factor as determinant of in vitro fertilization outcome. Hum Reprod, 7: 1136- 
1140.

2. Quinn P (1993): Sperm processing in assisted reproductive technology:Male factor. Semin Rep Endocrin, 11: 49-55.

3. Enginsu ME, Dumoulin JCM, Pieters MHEC, Evers JLH, Geraedts JPM (1993): Predictive value of morphologically normal sperm concentration in the medium for in-vitro fertilization. Int J Androl, 16: 113-120.

4. Calderon G, Veiga A, Torello MJ, Vidal E, Martinez F, Barri PN (1991): Do Percoll gradients improve sperm morphology. Hum Reprod, 6(Suppl): 39.

5. Cummins JM, Yovich JM. Sperm motility enhancement in vitro (1993): Semin Rep Endocrin, 11: 1-16.

6. Tash JS and Means AR (1983): Cyclic adenosine 3',5'-monophosphate, calcium and protein phosphorylation in flagellar motility. Biol Reprod, 28: 75-104.

7. Chan YM, Chan SY, Tucker MJ, Wong CJ, Leung CK, Leong MK (1990): Effects of dibutyryl cyclic guanosine monophosphate on human spermatozoal motility and penetration of zona-free hamster oocytes. Hum Reprod, 5: 304-308.

8. Perreault SD, Rogers BJ (1982): Relationship between fertilizing ability and cAMP in human spermatozoa. J Androl, 3: 396-401.

9. Aitken RJ, Mattei A, Irvine S (1986): Paradoxical stimulation of human sperm motility by 2-deoxyadenosine. J Reprod Fert, 78: 515-527.

10. Aparicio NJ, Schwarztein L, de Turner EA (1980): Pentoxifylline (BL 191) by oral administration in the treatment of asthenozoospermia. Andrologia, 12: 228-231.

11. Marrama P, Baraghini GF, Carani C, Celani MF, Giovenco P, Grandi F, Montanini V (1985): Further studies on effect of Pentoxifylline on sperm count and sperm motility in patients with idiopathic oligo-asthenozoospermia. Andrologia, 17: 612616.

12. Shen Meng Ru, Chiang PH, Yang RC, Hong CY, Chen SS (1991): Pentoxifylline stimulates human sperm motility both in vitro and after oral therapy. $\mathrm{Br} \mathrm{J}$ Clin Phar, 31: 711-714.

13. Sikka SC, Hellstrom WJ (1991): The application of pentoxifylline in the stimulation of sperm motion in men undergoing electroejaculation. J Androl, 12: 165-170.

14. Yovich JM, Edirisinghe WR, Cummins JM, Yovich JL (1988): Preliminary results using pentoxifylline in a pronuclear stage tubal transfer (PROST) program for severe male factor infertility. Fertil Steril, 50: 179-181.

15. Yovich JM, Edirisinghe WR, Cummins JM, Yovich JL (1990): Influence of pentoxifylline in severe male factor infertility. Fertil Steril, 53: 715-722.

16. Tournaye H, Staessen C, Janssens R, Devroey-P, Camus M, Van Steirteghem AC (1993): Pentoxifylline is not useful in enhancing sperm function in cases with previous in vitro fertilization failure. Fertil Steril, 59: 210-215.

17. Quinn P, Kerin JF, Warnes GM (1985): Improved pregnancy rate in human in vitro fertilization witht he use of a medium based on the composition of human tubal fluid. Fertil Steril, 44: 493-498.

18. Fleiss JL (1988): The design and analysis of clinical experiments. Wiley. New York.

19. Tesarik J, Thebault A, Testart J (1992): Effect of pentoxifylline on sperm movement characteristics in normospermic and asthenospermic specimens. Hum Reprod, 7: 1257-263.

20. Moohan JM, WinstonRML, Lindsay KS (1993): Variability of human sperm response to immediate and prolonged exposure to pentoxifylline. Hum Reprod, 8: 1696700.

21. Wang R, Bell M, Sikka SC, Hellstrom WJ, Veeraragavan K (1993): Platelet activating factor and pentoxifylline as human sperm cryoprotectants. Fertil Steril, 
60: 711-715.

22. Fraser LR, Monks NJ (1990): Cyclic nucleotides and mammalian sperm capacitation. J Rep Fert Suppl, 42:9-21.

23. DasGupta S, Mills C, Fraser LR (1993): Pentoxifylline promotes capacitation inhuman spermatozoa as assessed by a CTC fluorescence assay. Abstract book of International symposium on male infertility and assisted reproduction. Paper 135.

24. De Jonge CJ, Han HL, Mack SR, Zaneveld LJD (1991): Modulation of the human sperm acrosome reaction by effectors of the adenylate cyclase/cyclic AMP secondmessenger pathway. J Exp Zool, 258: 113-125.

25. Tesarik J, Mendoza C, Carreras A (1992): Effects of phosphodiesterase inhibitors caffeine and pentoxifylline on spontaneous and stimulus-induced acrosome reactions in human sperm. Fertil Steril, 58: 1185-1190.

26. Hammitt DG, Bedia E, Rogers PR, Syrop CH, Donovan JF, Williamson RA (1989): Comparison of motility stimulants for cryopreserved human semen. Fertil Steril, 52: 495-502.

27. Pang SC, Chan PJ, Lu A (1993): Effects of pentoxifylline on sperm motility and hyperactivation in normozoospermic and normokinetic semen. Fertil Steril, 60 : 336-343.

28. Tesarik J, Mendoza C (1993): Sperm treatment with pentoxifylline improves the fertilizing ability in patients with acrosome reaction insufficiency. Fertil Steril, 60: 141-148.

29. Siracusa G, De Felici M, Salustri A (1990): Meiotic maturation of the mammalian oocyte. In Asch RH, Balmaceda JP and Johston I (eds), Gamete Physiology. Serono Symposia USA, Norwell, MA, USA, pp. 129-144. 


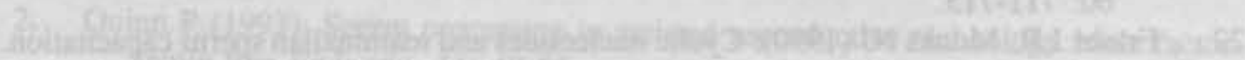

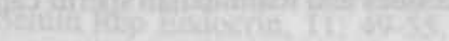

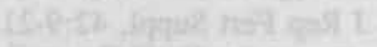

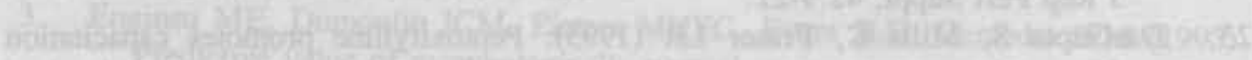

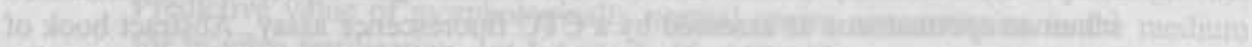

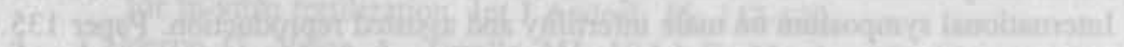

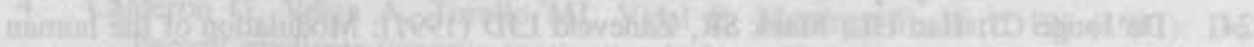

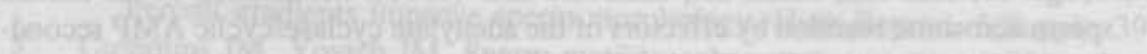

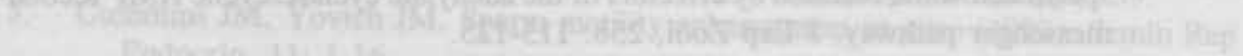

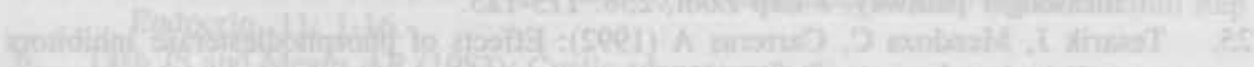

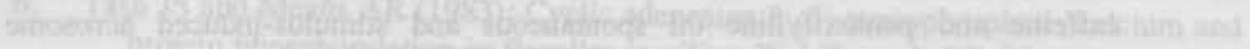

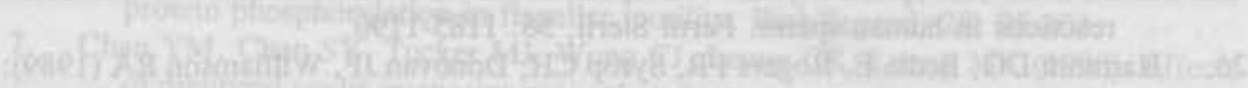

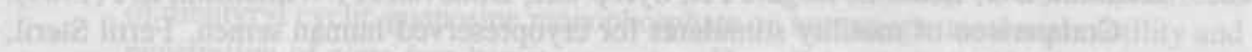

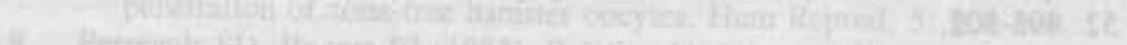

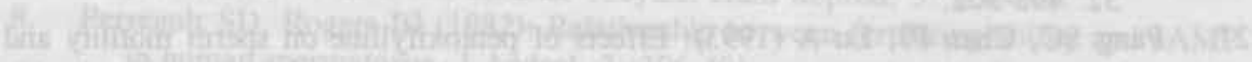

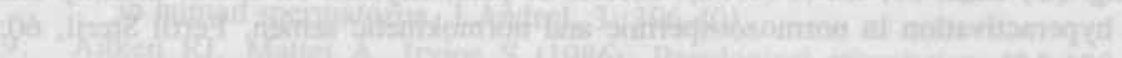

9i

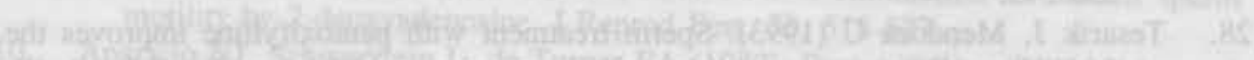

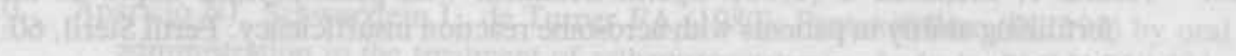

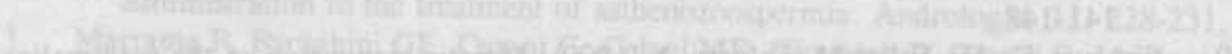

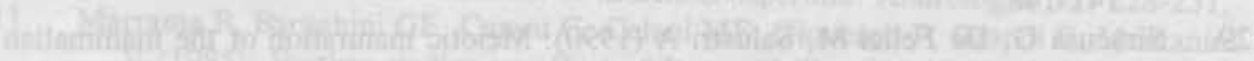

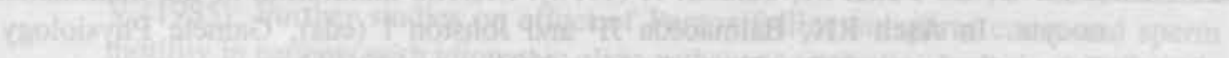
106

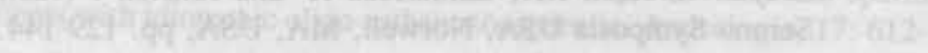

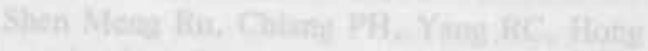

-

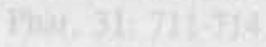

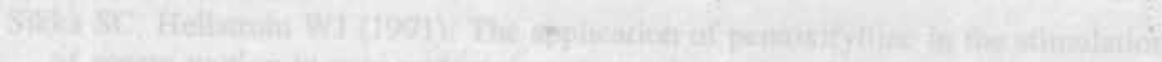

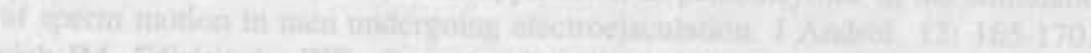

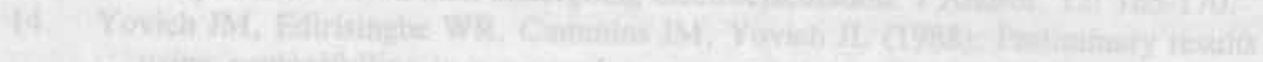

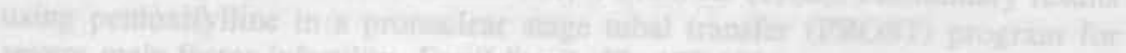

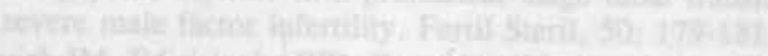

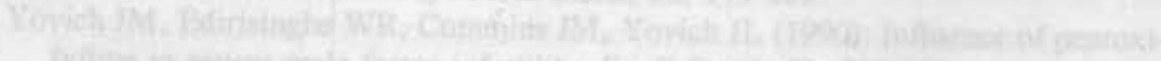

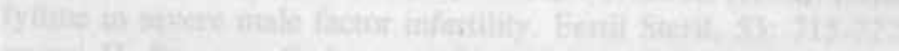

10.

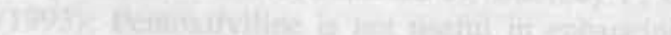

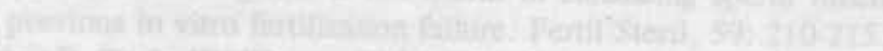

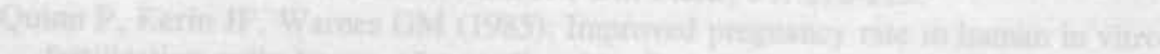

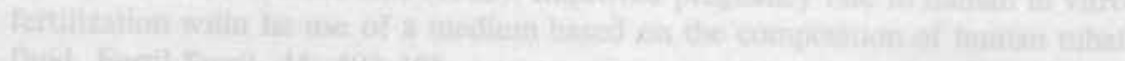

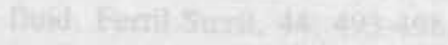

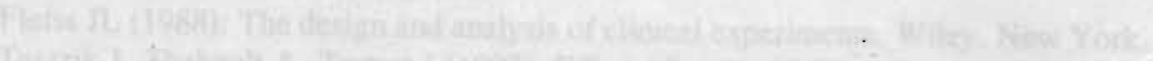

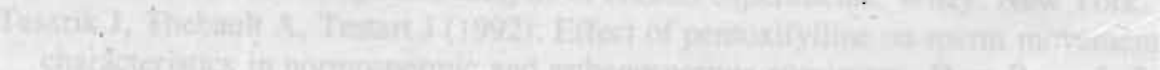

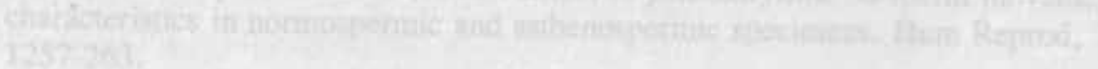

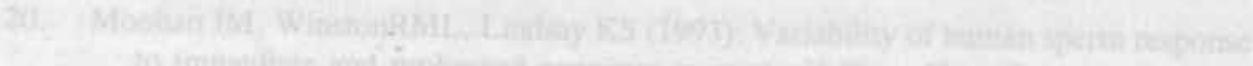

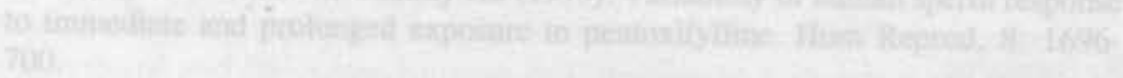

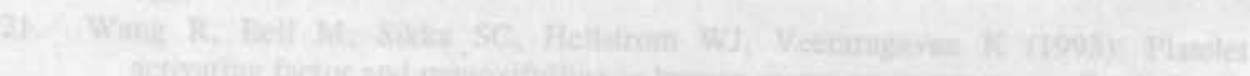

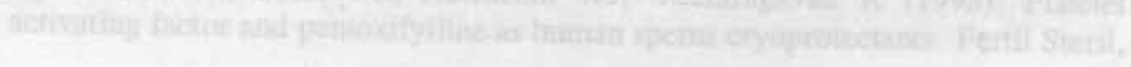




\section{General Discussion}

The terms fertility and infertility suggest a binomial partition of patients into disease and health categories which in reality does not exist. Infertility, defined as the inability of a couple to establish a pregnancy, is rare. In the Netherlands, it occurs in about $3 \%$ of couples (primary infertility), whereas an estimated $6 \%$ do not succeed in obtaining the number of children desired (secondary infertility)(3). This may be caused by absolute infertility (i.e. sterility) of the man (e.g. due to azoospermia), or of the woman, e.g. due to untimely depletion of the ovarian follicular content (POF, premature ovarian failure). In most couples visiting fertility clinics however, more contestable causes of impaired fertility will be encountered: e.g. subnormal sperm counts (oligozoospermia) or failure of the ovarian follicle to rupture (LUF, Luteinized Unruptured Follicle syndrome) in some (but not all!) of the menstrual cycles. For these cases of decreased monthly fecundity the term subfertility is to be preferred. If for example both latter mentioned causes of decreased fertility are present in a couple, only a small chance exists of the man having a flare up of his sperm count exactly on the right day of that particular menstrual cycle of his partner that is not characterized by a failure of the follicle to rupture. Eventually however, with time, this opportunity will materialize and conception will occur. Investigation of both partners therefore is imperative in case of fertility problems, and assessment of the individual and combined fertility potential is of utmost importance.

Traditionally, the woman has been the focal point of the fertility investigation. Hence the relegation of this field of clinical medicine to the specialty of gynaecology. There exists growing concern about (declining?) fertility, and potential male causes of decreased fertility have been receiving more attention recently. Andrology is emerging as a subspecialty of its own. Evidence is accumulating that at least half of the couples seeking reproductive assistance are suffering from a male problem (22).

In vitro fertilization (IVF) has opened a new era in the treatment of the infertile couple. Also, IVF has enabled the scientist to gain more insight in gamete interaction. Since the spermatozoa have to be separated from the seminal plasma before they can capacitate and undergo acrosome reaction (which are prerequisites for fertilization), sperm processing became an integral part of the routine IVF laboratory procedure. After sperm processing either the density alone or the density of the motile fraction of the spermatozoa of the final suspension is adjusted prior to insemination. This means that at most two of the major sperm parameters, density and motility, are adjusted. The role of the third, morphology was therefore the main subject of this thesis.

When human spermatozoa in the semen come into contact with cervical mucus, 
only a small fraction succeeds in penetration (18). When the morphology of this portion was evaluated, both after in vivo (9) and in vitro penetration (6), it was found that the morphologically abnormal spermatozoa were less able to penetrate the mucus than their morphologically normal counterparts.

Morales and coworkers (15) have found that morphologically normal spermatozoa swim faster than abnormal spermatozoa. Normal spermatozoa also have higher rolling and flagellar beat frequencies and flagellar amplitude (15).

Sperm Penetration Assay (SPA) and Hemi Zona Assay (HZA) are two diagnostic tests used for the detection of abnormality of male origin. The results of these tests have shown that normal morphology correlates highly with positive test results, suggesting that morphologically normal spermatozoa have a better chance of binding to the human zona pellucida and of forming a male pronucleus in a hamster oocyte $(13,16)$.

These results support the notion that the motility of the spermatozoa is highly related to their morphology. Therefore, in ART (Assisted reproductive technologies), morphology evaluation may become the cornerstone of the semen analysis.

A reliable morphology evaluation technique begins with well prepared slides and a staining technique (20). Papanicolaou staining is widely used for this purpose. However, the time spent for this procedure (approximately $1 \mathrm{~h}$ ) causes a shrinkage of the sperm head due to its exposure to the fixative and staining solutions. When living sperm heads of native samples were compared with their Papanicolaou stained counterparts, the measurements of the stained sperm head were in the $95 \%$ confidence intervals of the native sperms (10). For the DiffQuik staining technique, on the other hand, spermatozoa are exposed to the fixative and to two staining solutions for a total of 30 seconds. Since the shrinkage problem is eliminated with this technique, the morphology of the sperm head can be evaluated more reliably. Furthermore, the latter technique is time saving and the background of the slides gives better contrast.

Evaluation of human sperm morphology has been subjected to much debate after the introduction of new evaluation criteria by Kruger and co-workers (12). These new criteria were based on the morphology of the spermatozoa that can penetrate cervical mucus and bind to the zona pellucida. In the present thesis, these new criteria are studied: morphology evaluation using strict criteria (MEUSC). The main differences between the MEUSC and the WHO criteria are that in MEUSC all borderline forms are considered abnormal and the sperm head should have a smooth oval shape. The first part of this thesis is devoted to the selection of the parameter that is a good predictor of IVF results. 
Table 1. The likelihood ratios of semen parameters with the $95 \%$ confidence intervals.

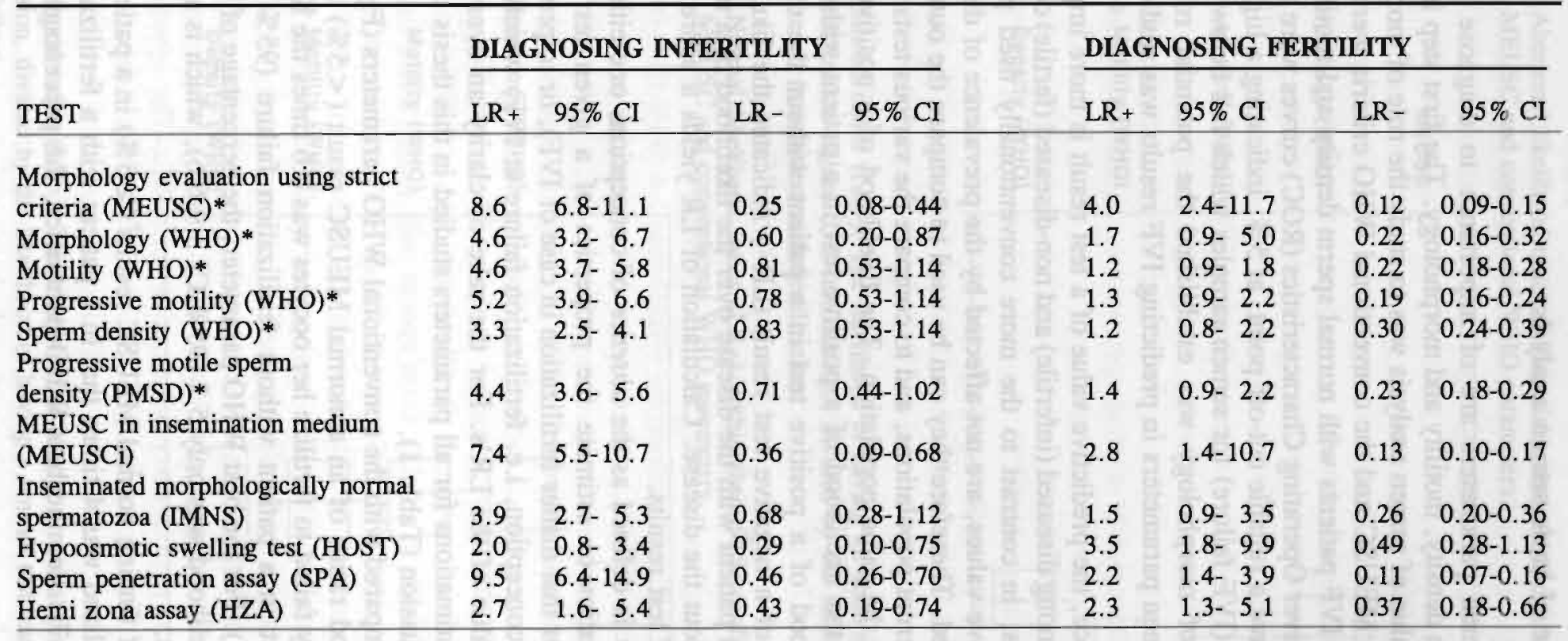

* These parameters have been used in several studies in this thesis. Therefore, a homogeneous group is made, discarding the overlapping results. 


\section{Diagnostic value of basic semen analysis}

Three basic semen parameters are of importance to diagnose male factor infertility: sperm density, motility and morphology. The first step in evaluating the diagnostic value of semen analysis was to study the role of morphology. In Chapter II, both MEUSC and the conventional WHO criteria were simultaneously applied in IVF patients with normal sperm density and motility parameters. When Receiver Operating Characteristics (ROC) curves were constructed, only MEUSC gave a reliable cut-off point, at $5 \%$, indicating a high chance of $0 \%$ fertilization (IVF failure) for semen samples which fall below this point. Once the role of morphology was established the potential role of other conventional semen parameters in predicting IVF results was studied (Chapter III to V).

In clinical practice, the predictive value of a test result is more important than its distribution among diseased (infertile) and non-diseased (fertile) couples (17). Likelihood ratios, in contrast to the more conventionally used positive and negative predictive values, are not affected by the prevalence of disease in the population studied. Therefore they can be used to compare the outcome of the same test in different populations, and to compare the various tests of the same disease entity in the same population. The likelihood of a positive test result $(L R+)$ indicates the likelihood of a positive test in a patient with the disease over the likelihood of a positive test in a patient without the disease. The likelihood ratio of a negative test result (LR-) indicates the likelihood of a negative test in a patient with the disease over the likelihood of a negative test in a patient without the disease. Calculation of LR yields a score that allows categorization of test results.

Since subfertility is defined as the absence of conception, one either may use the likelihood ratios to estimate the properties of a given test to predict conception (or for that matter fertilization in case of IVF), or to predict disease (i.e. absence of conception, i.e. fertilization failure at IVF) which constitutes the reciprocal value of the LR's. For the sake of clarity an overview of the results of both estimations for all parameters studied in this thesis is offered in this general discussion (Table 1).

MEUSC was compared with the conventional WHO parameters (Figures 1 and 2 ). The likelihood ratios of an abnormal MEUSC result $(<5 \%)$ in a patient who subsequently failed to fertilize her oocytes was 8.6 times the likelihood of an abnormal test in a patient without fertilization failure $(95 \%$ confidence interval 6.8-11.1). For the best WHO parameter, the percentage of progressive motility, this likelihood was only 5.2 (95\% CI 3.9-6.6), which is significantly worse (Figure 1).

The likelihood of finding normal MEUSC results $(\geq 5 \%)$ in a patient who had her oocytes fertilized was 4 times that in a patient with a fertilization failure (95\% CI 2.3-11.7). For the best WHO parameter, the percentage of normal 
Figure 1. Abnormal likelihood ratios (LR+) within their 95\% confidence intervals, for MEUSC and conventional WHO parameters.

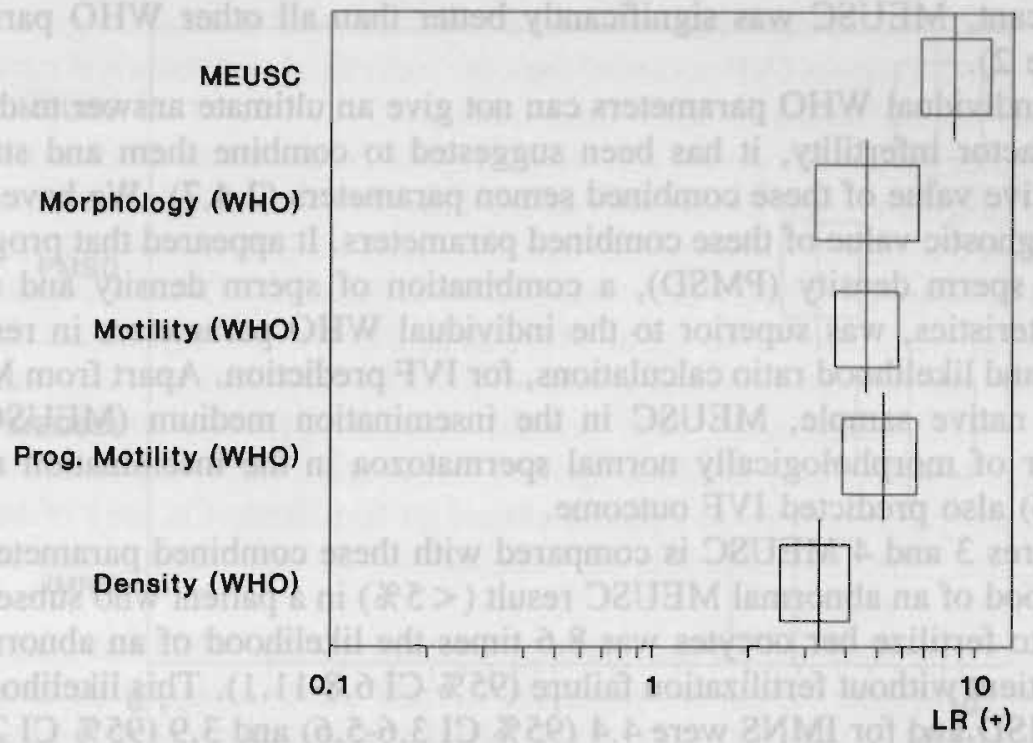

Abnormal likelihood ratios (LR+): The likelihood of having an abnormal test result, in a patient with fertilization failure ( $0 \%$ fertilization), divided by the likelihood of having an abnormal test result in a patient with fertilization.

Figure 2. Normal likelihood ratios (LR+) within their $95 \%$ confidence intervals for MEUSC and conventional WHO parameters.

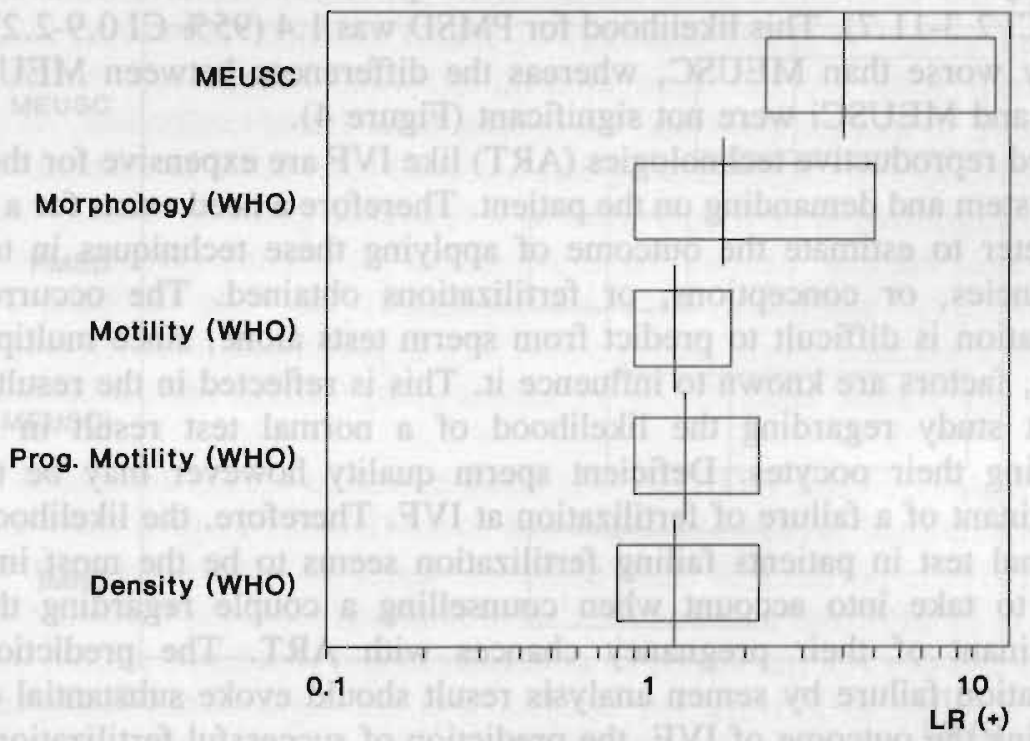

Normal likelihood ratios ( $L R+)$ : The likelihood of having a normal test result in a patient with fertilization, divided by the likelihood of having a normal test result in a patient with fertilization failure. 
morphology, this likelihood was 1.7 (95\% CI 0.9-5.0). Although the difference between these figures, for MEUSC and WHO morphology respectively, is not significant, MEUSC was significantly better than all other WHO parameters (Figure 2).

Since individual WHO parameters can not give an ultimate answer to diagnose male factor infertility, it has been suggested to combine them and study the predictive value of these combined semen parameters $(2,4,7)$. We have studied the diagnostic value of these combined parameters. It appeared that progressive motile sperm density (PMSD), a combination of sperm density and motility characteristics, was superior to the individual WHO parameters in respect to kappa and likelihood ratio calculations, for IVF prediction. Apart from MEUSC in the native sample, MEUSC in the insemination medium (MEUSCi) and number of morphologically normal spermatozoa in the insemination medium (IMNS) also predicted IVF outcome.

In figures 3 and 4 MEUSC is compared with these combined parameters. The likelihood of an abnormal MEUSC result $(<5 \%)$ in a patient who subsequently failed to fertilize her oocytes was 8.6 times the likelihood of an abnormal test in a patient without fertilization failure (95\% CI 6.8-11.1). This likelihood ratio for PMSD and for IMNS were 4.4 (95\% CI 3.6-5.6) and 3.9 (95\% CI 2.7-5.3) respectively, both of which performed significantly worse than MEUSC. The only parameter which did not show a significant difference was MEUSCi with a likelihood ratio of 7.4 (95\% CI 5.5-10.7) which was not significantly different from PMSD but was significantly better than IMNS (Figure 3).

The likelihood of finding normal MEUSC results $(\geq 5 \%)$ in a patient who had her oocytes fertilized was 4 times that in a patient with a fertilization failure (95\% CI 2.3-11.7). This likelihood for PMSD was 1.4 (95\% CI 0.9-2.2), significantly worse than MEUSC, whereas the differences between MEUSC and IMNS and MEUSCi were not significant (Figure 4).

Assisted reproductive technologies (ART) like IVF are expensive for the health care system and demanding on the patient. Therefore a need exists for a reliable parameter to estimate the outcome of applying these techniques in terms of pregnancies, or conceptions, or fertilizations obtained. The occurrence of fertilization is difficult to predict from sperm tests alone, since multiple, also female, factors are known to influence it. This is reflected in the results of the present study regarding the likelihood of a normal test result in patients fertilizing their oocytes. Deficient sperm quality however may be the sole determinant of a failure of fertilization at IVF. Therefore, the likelihood of an abnormal test in patients failing fertilization seems to be the most important factor to take into account when counselling a couple regarding the male determinant of their pregnancy chances with ART. The prediction of a fertilization failure by semen analysis result should evoke substantial concern regarding the outcome of IVF, the prediction of successful fertilization on the hasis of semen analysis results should always be considered with reserve. 
Figure 3. Abnormal likelihood ratios (LR+) within their $95 \%$ confidence intervals, for MEUSC and combined parameters.

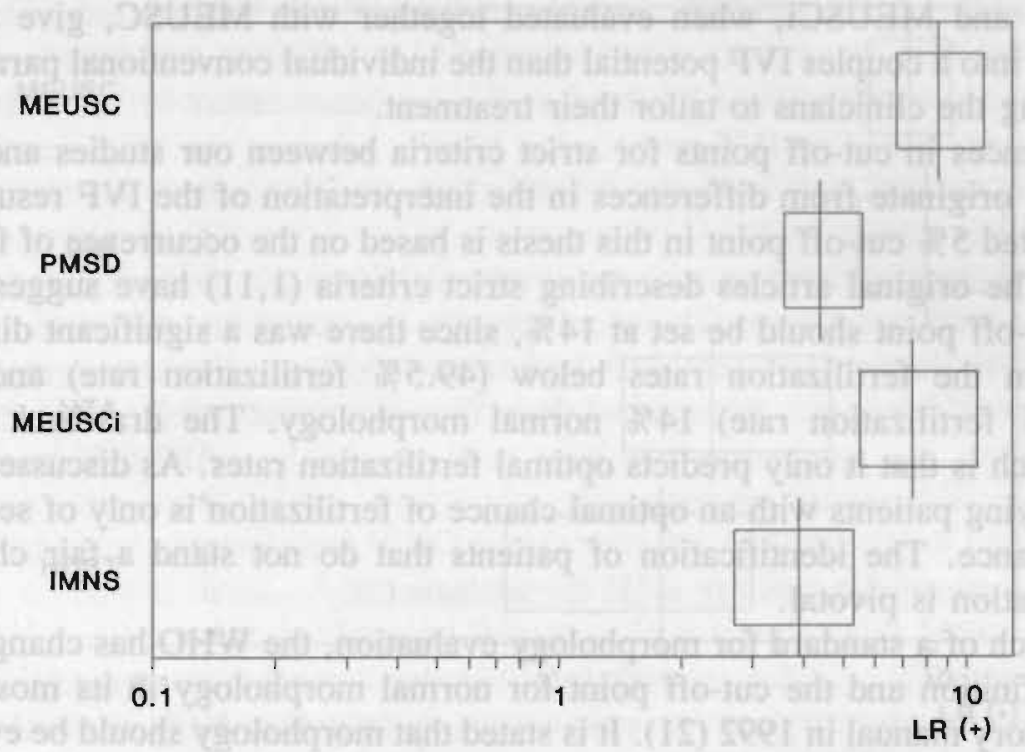

Abnormal likelihood ratios (LR+): The likelihood of having an abnormal test result, in a patient with fertilization failure $(0 \%$ fertilization), divided by the likelihood of having an abnormal test result in a patient with fertilization.

Figure 4. Normal likelihood ratios $(L R+)$ within their $95 \%$ confidence intervals for MEUSC and combined parameters.

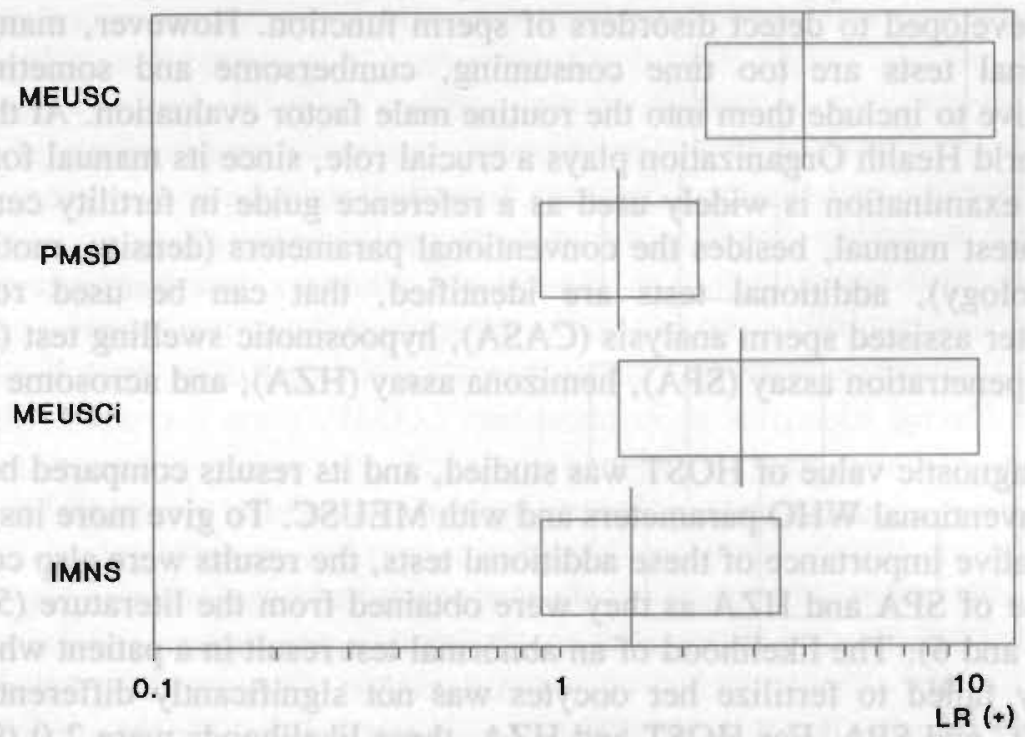

Normal likelihood ratios (LR+): The likelihood of having a normal test result in a patient with fertilization, divided by the likelihood of having a normal test result in a patient with fertilization failure. 
MEUSC is superior to semen analysis according to WHO criteria in detecting couples with an inadequate male factor. The additional parameters such as PMSD and MEUSCi, when evaluated together with MEUSC, give a better insight into a couples IVF potential than the individual conventional parameters, allowing the clinicians to tailor their treatment.

Differences in cut-off points for strict criteria between our studies and others $(1,11)$, originate from differences in the interpretation of the IVF results. The suggested $5 \%$ cut-off point in this thesis is based on the occurrence of fertilization. The original articles describing strict criteria $(1,11)$ have suggested that the cut-off point should be set at $14 \%$, since there was a significant difference between the fertilization rates below $(49.5 \%$ fertilization rate) and above ( $88.3 \%$ fertilization rate) $14 \%$ normal morphology. The drawback on this approach is that it only predicts optimal fertilization rates. As discussed above identifying patients with an optimal chance of fertilization is only of secondary importance. The identification of patients that do not stand a fair chance at fertilization is pivotal.

In search of a standard for morphology evaluation, the WHO has changed both the definition and the cut-off point for normal morphology in its most recent laboratory manual in 1992 (21). It is stated that morphology should be evaluated strictly. The normal sperm should have an oval shape and all borderline forms should be considered abnormal. This definition perfectly fits with the strict criteria. The cut-off point was also lowered from $50 \%$ to $30 \%$ morphologically normal spermatozoa. Thirty percent normal morphology is still higher than the $14 \%$ (for WHO criteria) suggested in the present study, based on ROC curves. Besides the descriptive basic semen analysis, additional diagnostic tests have been developed to detect disorders of sperm function. However, many of the additional tests are too time consuming, cumbersome and sometimes too expensive to include them into the routine male factor evaluation. At this point the World Health Organization plays a crucial role, since its manual for human semen examination is widely used as a reference guide in fertility centers. In their latest manual, besides the conventional parameters (density, motility and morphology), additional tests are identified, that can be used routinely: computer assisted sperm analysis (CASA), hypoosmotic swelling test (HOST), sperm penetration assay (SPA), hemizona assay (HZA), and acrosome reaction tests.

The diagnostic value of HOST was studied, and its results compared both with the conventional WHO parameters and with MEUSC. To give more insight into the relative importance of these additional tests, the results were also compared to those of SPA and HZA as they were obtained from the literature $(5,14)$ (Figure 5 and 6). The likelihood of an abnormal test result in a patient who subsequently failed to fertilize her oocytes was not significantly differentbetween MEUSC and SPA. For HOST and HZA, these likelihoods were $2.0(95 \% \mathrm{CI}$ $0.8-3.4)$ and 2.7 (95\% CI 1.6-5.4) respectively, which were significantly worse 
Figure 5. Abnormal likelihood ratios $(L R+)$ within their $95 \%$ confidence intervals, for MEUSC and additional tests.

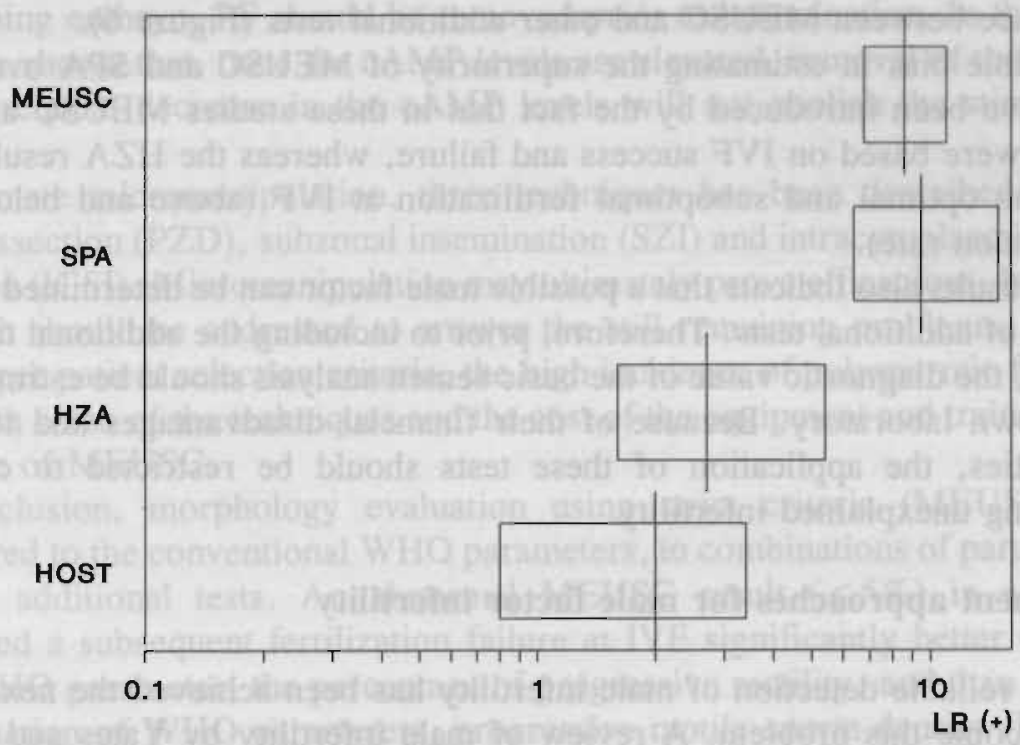

Abnormal likelihood ratios (LR+): The likelihood of having an abnormal test result, in a patient with fertilization failure ( $0 \%$ fertilization), divided by the likelihood of having an abnormal test result in a patient with fertilization.

Figure 6. Normal likelihood ratios $(L R+)$ within their $95 \%$ confidence intervals for MEUSC and additional tests.

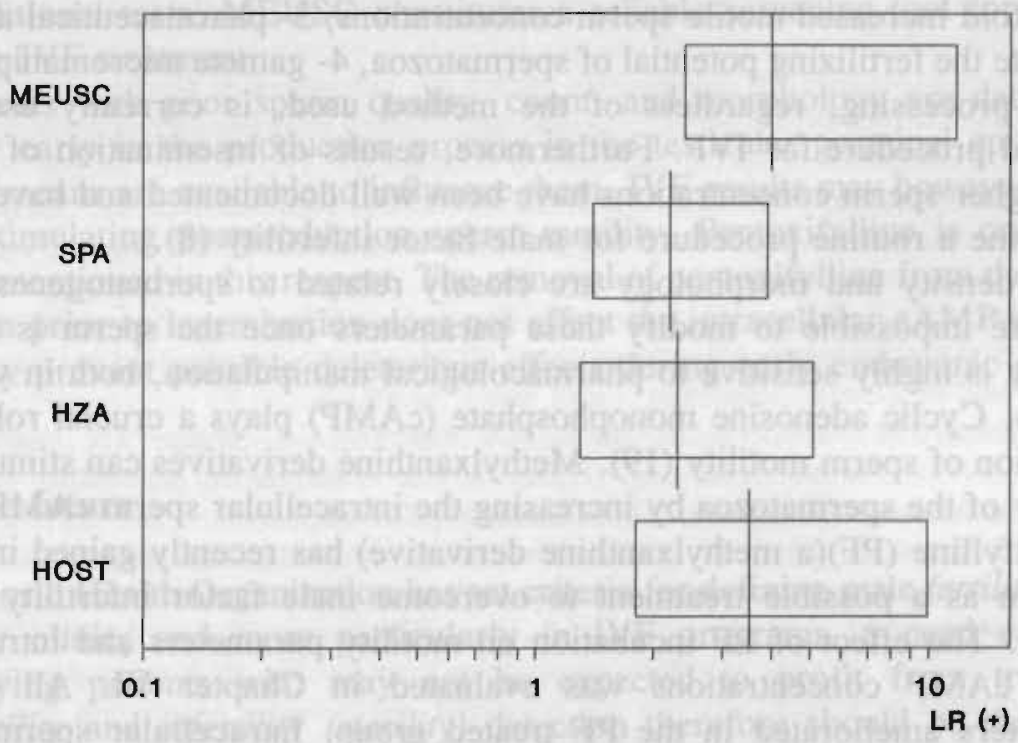

Normal likelihood ratios (LR+): The likelihood of having a normal test result in a patient with fertilization, divided by the likelihood of having a normal test result in a patient with fertilization failure. 
than both MEUSC and SPA (Figure 5). The likelihood of finding normal test results in a patient who had her oocytes fertilized did not show a significant difference between MEUSC and other additional tests (Figure 6).

A possible bias in estimating the superiority of MEUSC and SPA over HZA may have been introduced by the fact that in these studies MEUSC and SPA results were based on IVF success and failure, whereas the HZA results were based on optimal and suboptimal fertilization at IVF (above and below 55\% fertilization rate).

These results also indicate that a possible male factor can be determined without the use of additional tests. Therefore, prior to including the additional tests into routine, the diagnostic value of the basic semen analysis should be estimated for one's own laboratory. Because of their financial disadvantages and technical difficulties, the application of these tests should be restricted to cases of persisting unexplained infertility.

\section{Treatment approaches for male factor infertility}

Once a reliable detection of male infertility has been achieved the next step is to overcome this problem. A review of male infertility by Yates and Kretser (23) stresses the fact that IVF seems to be one of the most effective treatments for male-factor infertility. In fact, recent advances in ART focus on the male infertility problem. To overcome the male-factor infertility by ART, therapies are needed that will improve fertilization and pregnancy rates. For this purpose four types of laboratory techniques have been introduced: 1- sperm processing methods, i.e. swim-up, Percoll gradients, 2- insemination of oocytes with 10 to 100 fold increased motile sperm concentrations, 3- pharmaceutical agents to stimulate the fertilizing potential of spermatozoa, 4- gamete micromanipulation. Sperm processing, regardless of the method used, is currently used as a standard procedure for IVF. Furthermore, results of insemination of oocytes with higher sperm concentrations have been well documented and have started to become a routine procedure for male-factor infertility (8).

Sperm density and morphology are closely related to spermatogenesis. It is therefore impossible to modify these parameters once the sperm is formed. Motility is highly sensitive to pharmacological manipulation, both in vivo and in vitro. Cyclic adenosine monophosphate (cAMP) plays a crucial role in the regulation of sperm motility (19). Methylxanthine derivatives can stimulate the motility of the spermatozoa by increasing the intracellular sperm cAMP levels. Pentoxifylline (PF)(a methylxanthine derivative) has recently gained increased attention as a possible treatment to overcome male factor infertility in IVF $(24,25)$. The effect of PF incubation on motility parameters and intracellular sperm cAMP concentrations was evaluated in Chapter VI. All motility parameters ameliorated in the PF treated group. Intracellular sperm cAMP concentrations increased in the PF group immediately after incubation. 
However, following the removal of PF, intracellular sperm cAMP concentrations decreased rapidly. To avoid the possible toxic effects of PF on the developing embryo, PF should be removed prior to insemination. In this study we have shown that, once the cAMP levels are elevated, removal of the PF and the subsequent decrease in the cAMP levels will not abolish the stimulatory effect.

For gamete micromanipulation, three techniques has been described: partial zona dissection (PZD), subzonal insemination (SZI) and intracytoplasmic sperm injection (ICSI). Micromanipulation may ultimately prove efficacious, but much research should be addressed to answer the still remaining problems, such as the proper patient selection criteria, the high incidence of polyspermic fertilization with some of the techniques and the cost of the equipment and training, and the role of MEUSC.

In conclusion, morphology evaluation using strict criteria (MEUSC) was compared to the conventional WHO parameters, to combinations of parameters, and to additional tests. An abnormal MEUSC result $(<5 \%)$ in a patient predicted a subsequent fertilization failure at IVF significantly better than the best WHO parameter, the percentage of progressive motility, and than the best combination of WHO parameters, progressive motile sperm density (PMSD). Additional tests, notably hypoosmotic swelling test (HOST)and Hemi-Zona Assay (HZA), performed significantly worse than MEUSC in predicting fertilization failure at IVF. Sperm Penetration Assay (SPA) did better than the MEUSC, although not significantly. It is concluded that in view of the need for identifying poor prognosis patients, given the results of the present series of experiments, and taking into account the laboriousness and financial burden of the additional tests, MEUSC constitutes a reliable screening tool for couples seeking IVF treatment.

In patients with poor sperm quality, count, and morphology are determined already early in the production process in the testicular germinal epithelium, and no means are available to influence them. IVF results may however benefit from stimulating postproduction sperm motility. Pentoxifylline is one of the agents mentioned in this respect. The removal of pentoxifylline from the culture medium prior to insemination does not affect the intracellular cAMP increase, but may prevent possible deleterious effects during early embryonic development.

\section{For the future}

The World Health Organization has set criteria for defining male fertility. In the fertility clinic, and more particularly in IVF programs, a need exists for identifying patients who may not be expected to profit from treatment. Subfertility and infertility (sterility) detection therefore should be the aim of future WHO efforts in this respect. At the moment this pertains to identifying 
those patients who may not benefit from IVF. With the recent introduction of Intra Cytoplasmic Sperm Injection (ICSI) technique straight into the oocyte, the need will arise for methods allowing identification of patients who will not benefit from regular IVF, but neither from this microfertilization method. Also here the potential of MEUSC deserves consideration.

Not only in assisted reproduction, but also in the routine fertility investigation a need exists for reliable methods to assess a couple's fecundability. MEUSC also may offer a promising alternative to WHO criteria in predicting in vivo fertilization. Therefore, it seems worthwhile to investigate the value of MEUSC as a screening tool in the routine fertility clinic. Such studies of course can not resort to fertilization as an endpoint and therefore will have to rely on pregnancy rates in large groups prospectively. This will require the application of demographical and epidemiological techniques.

Male fertility has not been devoted much medical attention for ages. Since the appreciation of the male contribution to a couple's reproduction, gradually diagnostic tests for assessing male fertility have become available. Treatment options however, are extremely limited. The introduction of micro fertilization techniques definitely will allow more male fertility problems to be treated. This however, only will be possible at the expense of a considerable amount of active involvement of his female partner. The availability of a reliable method for gauging the fertilizing capacity of human spermatozoa will be of vital importance in this respect.

\section{References}

1. Acosta AA, Oehninger S, Morshedi M, Swanson RJ, Scott R, Irianni F (1988): Assisted reproduction in the diagnosis and treatment of the male factor. Obstet Gynecol Survey, 44:1-18.

2. Cohen J, Alikani M, Malter HE, Adler A, Talansky BE, Rosenwaks Z (1991): Partial zona dissection or sub zonal insemination: microsurgical alternatives based on evaluation of sperm and embryo morphology. Fertil Steril, 56:696-706

3. Evers JLH, te Velde ER (1993): Vruchtbaarheidsstoornissen. In: Obstetrie en Gynaecologie: De voortplanting van de mens. Treffers PE (ed.), Wetenschappelijke uitgeverij Bunbe, Utrecht. Pg. 463.

4. Francavilla F, Romano R, Santucci R, Poccia G (1990): Effect of sperm morphology and motile sperm count on outcome of intrauterine insemination in oligospermia and/or asthenozoospermia. Fertil Steril, 53:892-7.

5. Franken DR, Lombard CJ, Acosta AA, Oehninger S, Kruger TF, Hodgen GD (1993): The hemizona assay (HZA): its role in identifying male factor infertility in assisted reproduction. Fertil Steril, 59:1075-80.

6. Fredricsson B, Bjork G (1977): Morphology of post coital spermatozoa in the cervical secretion and its clinical significance. Fertil Steril 28(8):841-5.

7. Glazener CMA, Kelly NJ, Weir MJA, Davis JSE, Cornes JS, Hull MGR (1987): The diagnosis of male infertility-prospective time specific study of conception rates related to seminal analysis and postcoital sperm-mucus penetration and survival in otherwise unexplained infertility. Hum Reprod, 2:665-71.

8. Hammit DG (1993): Treatment of male-factor infertility by in vitro insemination with 
high concentrations of motile sperm. Semin Rep Endocrin, 11:72-82.

9. Hanson FW, Overstret JW (1981): The interaction of human spermatozoa with cervical mucus in vivo. Am J Obs Gyn, 140:173.

10. Katz DF, Overstreet JW, Samuels SJ, Niswander PW, Bloom TD, Lewis EL (1986): Morphometric analysis of spermatozoa in the assessment of human male infertility. J Androl, 7:203-10.

11. Kruger TF, Acosta AA, Simmons KF, Swanson RJ, Matta JF, Veeck LL, Morshedi M, Brugo S (1987): New method of evaluating sperm morphology with predictive value for human in vitro fertilization. Urology, 30:248-251.

12. Kruger TF, Acosta AA, Simmons KF, Swanson JR, Matta JF, Oehniger S (1988): Predictive value of abnormal sperm morphology in in vitro fertilization. Fertil Steril, 49:112-117.

13. Kruger TF, Swanson RJ, Hamilton M, Simmons KF, Acosta AA, Matta JF, Oehniger S, Morshedi M (1988): Abnormal sperm morphology and other semen parameters related to the outcome of the hamster oocyte human sperm penetration assay. Int $\mathrm{J}$ Androl, 11:107.

14. Margalioth EJ, Navot D, Laufer N, Lewin A, Rabinowitz R, Schenker JG (1986): Correlation between the zona-free hamster egg sperm penetration assay and human in vitro fertilization. Fertil Steril, 45:665-70.

15. Morales P, Overstreet JW, Katz DF (1988): Changes in human sperm motion during capacitation in vitro. J Reprod Fertil 83:119-28.

16. Oehninger S, Coddington CC, Scott R, Franken DR, Burkman LJ, Acosta AA, Hodgen GD (1989): Hemizona assay: assessment of sperm dysfunction and prediction of IVF outcome. Fertil Steril, 51:665.

17. Overstreet JW, Katz DF (1981): Sperm transport and capacitation, in Sciarra JJ (ed): Gynecology and Obstetrics, vol 5, ch. 45. New York, Harper \& Row, pp 1-10.

18. Settlage DSF, Motoshima M, Tredway DR (1973): Sperm transport from the external os to the fallopian tubes in women: a time and quantitation study. Fertil Steril 24:655.

19. Tash JS and Means AR (1983): Cyclic adenosine 3',5'-monophosphate, calcium and protein phosphorylation in flagellar motility. Biol Reprod, 28:75-104.

20. WHO (1987) Laboratory Manual for the Examination of Human Semen and SpermCervical Mucus Interactions. Cambridge, Cambridge University Press, 2nd edn, pg 9-11.

21. WHO (1992) Laboratory Manual for the Examination of Human Semen and SpermCervical Mucus Interactions. Cambridge, Cambridge University Press, 3rd edn, pg 14.

22. WHO manual for the standardized investigation and diagnosis of the infertile couple (1993). Rowe PJ, Hargreave TB, Mellows HJ, Comhaire FH (eds), Cambridge, Cambridge University Press.

23. Yates CA, and Kretser DM (1987): Male-factor infertility and in vitro fertilization. J IVF ET, 4:141-147.

24. Yovich JM, Edirisinghe WR, Cummins JM, Jovich JL (1990): Influence of pentoxifylline in severe male factor infertility. Fertil Steril, 53:715-22.

25. Tesarik J, Mendoza C (1993): Sperm treatment with pentoxifylline improves the fertilizing ability in patients with acrosome reaction insufficiency. Fertil Steril, $60: 141-8$. 

The assessment of semen quality has long been based on sperm density, motility and morphology. In the last 5 years, sperm morphology has received increasing attention. This can be attributed to a new evaluation technique of morphology, which was introduced by Kruger and coworkers in 1988. At the same time a new staining technique, Diff-Quik, was introduced. This Diff-Quik staining is a time saving procedure compared to Papanicolaou staining which has also been widely used in evaluating sperm morphology. Slides stained with Papanicolaou and Diff-Quik have almost the same quality, enabling the morphological status of spermatozoa to be evaluated in detail. However, the latter technique gives a clear background of the stained smears.

The main difference between studies of morphology lies in the application of the criteria for evaluation. The predictive value of human sperm morphology for IVF was evaluated. For the first time, the WHO (World Health Organization) and Kruger's strict criteria (MEUSC) were compared in patients with normal sperm density and motility parameters. Although there was a correlation between the percentage of normal morphology with the WHO criteria and fertilization in-vitro $(r=0.282, p<0.001)$, the correlation for strict criteria was much better $(\mathrm{r}=0.555, \mathrm{p}<0.000005)($ Chapter II).

We have also determined an optimal cut-off point for normal morphology. Below 5\% normal morphology there was a severe impairment of the fertilization rate $(23 \%)$. Also in comparison to other sperm parameters, MEUSC showed the highest (and the only clinically acceptable) kappa value (0.439)(Chapter II).

In Chapter III, the predictive value of the Hypoosmotic Swelling Test (HOST) was evaluated and compared with the morphology scores.

The most appropriate cut-off point for the HOST in the native sample was found at $60 \%$ swelling. However, the kappa value of 0.151 demonstrates that this agreement was entirely due to chance. The correlation between sperm swelling in response to hypoosmotic conditions and fertilization in vitro was low $(r=0.334)$. MEUSC on the other hand correlated better with the fertilization outcomes and also predicted IVF failure better than the HOST(Chapter III).

In Chapter IV, a larger and nonselected IVF population was studied for both the WHO criteria and the strict criteria. The aim was to search for other parameters in the native sample to allow establishing reliable patient selection criteria for IVF. Of the classical seminal measurements, the best cut-off points to predict IVF failure were found to be: sperm density $20 \times 10^{6} / \mathrm{ml}$, motility $30 \%$, progressive motility $30 \%$, progressive motile sperm density (PMSD) $3 \mathrm{x}$ $10^{6} / \mathrm{ml}$, normal morphology using WHO criteria $14 \%$, and morphology evaluation using strict criteria $5 \%$. Even in this unselected IVF population, the cut-off point for the WHO criteria was below the official $50 \%$ WHO limits, which was applicable at the time of the study (Recently the WHO has changed 
the normal morphology lower limit to $30 \%$ ).

Since PMSD was the second best parameter, it was combined with MEUSC in predicting fertilization and pregnancy rates. When both parameters were below their respective cut-off points, the fertilization rate was only $18 \%$ and no pregnancies were achieved. On the other hand, the fertilization rate was $72 \%$ and the pregnancy rate was $27 \%$ if both parameters were above their respective cut-off points.

In Chapter V, morphology scores after swim-up and the number of morphologically normal spermatozoa in the insemination medium (IMNS) were evaluated with respect to IVF outcome.

The highest correlation between the pre- and post swim-up sperm parameters was found for sperm morphology scores, showing that sperm morphology score in the swim-up portion can be predicted from the native sample $(r=0.804)($ Chapter V).

After the swim-up procedure, a significant increase in spermatozoa exhibiting progressive motility and normal morphology was observed for the whole group. However, the percentage of spermatozoa with progressive motility did not improve significantly in the infertile group whereas in the fertile group there was a significant increase $(p<0.0005)$. The percentage of normal morphology showed a significantincrease in both groups $(p<0.001)$.

Since the number of progressive motile spermatozoa was adjusted to the same level in the IVF medium, the possible parameters to be used as IVF predictors were sperm density, the percentage of spermatozoa with normal morphology in the native sample and the number of morphologically normal spermatozoa used for IVF (IMNS). Sperm density had no effect on the IVF outcome. IMNS showed a weak correlation with fertilization outcome $(r=0.336, p<0.001)$ but failed to give a clinically acceptable cut-off point whereas MEUSC was the best predictor (Chapter IV).

The highest predictive value for IVF outcome was found for the percentage of morphologically normal spermatozoa, both in the native and post swim-up samples. Moreover, only these two parameters gave clinically acceptable cut-off points (5\% and $8 \%$ respectively). When native MEUSC scores and post swimup MEUSC scores were both below their respective cut-off levels, fertilization rate was $7 \%$ and no pregnancies were achieved.

The number of progressively motile spermatozoa can be elevated by motility stimulants. Pentoxifylline (PF), a phosphodiesterase inhibitor, is widely used for this purpose. In Chapter VI, the effects of PF incubation on intracellular cAMP concentrations and motility parameters have been evaluated. All motility parameters were significantly higher in the PF group when compared with the control group $(p<0.05)$. Intracellular cAMP concentrations were not significantly different in control and PF groups after processing of the spermatozoa as for IVF. Moreover, there was a significant elevation in the intracellular cAMP concentrations in the PF group immediately after incubation 
$(\mathrm{p}<0.0005)$. Our results are in agreement with previous findings and show that once the cAMP levels have been elevated with pentoxifylline, subsequent washing does not affect the hyperactivation and sensitization of the spermatozoa to spontaneous acrosome reaction, although the cAMP levels decrease. Therefore, PF can be used as a stimulant for IVF to enhance the progressive motile density and further washing will not negate the stimulatory effect. In conclusion, the results from Chapters II to IV show that morphology evaluation using strict criteria (MEUSC) for evaluation of human sperm morphology plays an important role in predicting IVF results and that it is superior to WHO criteria. Additional parameters for IVF prediction are PMSD in the native sample and the MEUSC in the insemination medium. Furthermore, the fact that there were no pregnancies in patients who have semen samples that fall below cut-off points for MEUSC, PMSD and post swim-up MEUSC scores, should motivate the clinician to search for treatment procedures in addition to classical IVF treatment, such as incubating the sperm with pentoxifylline, which enable the recovery of higher numbers of progressive motile spermatozoa for in vitro insemination (Chapter VI). 
De kwalitatieve beoordeling van semen is lang gebaseerd geweest op spermatozoaconcentratie, -motiliteit en -morfologie. Gedurende de laatste vijf jaar heeft de morfologie van de spermatozoa stteds meer de aandacht met name gekregen. Dit kan worden toegeschreven aan een nieuwe evaluatiemethode van de morfologie, die geïntroduceerd werd door Kruger en medewerkers in 1988. Tegelijkertijd werd door deze groep een nieuwe kleurtechniek geïntroduceerd. Deze Diff-Quik kleuring is een tijdbesparende procedure vergeleken met de veel gebruikte Papanicolaou kleuring. Beide kleurmethoden geven kwalitatief zeer goede en vergelijkbare preparaten waarbij het morfologisch aspect van spermatozoa tot in details bestudeerd kan worden. De Diff-Quik kleuring geeft echter minder achtergrondkleuring, hetgeen een voordeel is.

Het belangrijkste verschil tussen studies aangaande de morfologie van spermatozoa is gelegen in de toepassing van de evaluatiecriteria. In hoofdstuk II wordt de voorspellende waarde van de morfologie van humane spermatozoa onderzocht ten aanzien van in-vitro fertilisatie (IVF) resultaten. Voor het eerst sinds de introductie van de nieuwe evaluatiecriteria door Kruger en medewerkers werden de WHO (World Health Organization) en de strikte criteria van Kruger (MEUSC) met elkaar vergeleken bij patiënten met normale spermatozoaconcentraties en -motiliteit. De morfologie werd geëvalueerd in met Diff-Quik gekleurde preparaten, gemaakt van vers semen. Hoewel het percentage spermatozoa met normale morfologie volgens de WHO criteria bleek te correleren met de kans op bevruchting in vitro $(r=0.282, p<0.001)$, bleek deze correlatie bij de strikte criteria veel beter te zijn $(r=0.555, \mathrm{p}<0.000005)$ (Hoofdstuk II). Ook werd het optimale afkappunt voor normale morfologie bepaald. Bij semen met minder dan 5\% spermatozoa met normale morfologie (MEUSC) werd een laag bevruchtingspercentage gevonden $(23 \%)$. In vergelijking met andere semenparameters bleek de MEUSC de hoogste (en de enige klinisch acceptabele) kappa waarde $(0.439)$ te hebben (Hoofdstuk II).

In hoofdstuk III wordt de voorspellende waarde van de Hypoosmotic Swelling Test (HOST) ten aanzien van de bevruchting in vitro geëvalueerd, en vergeleken met de morfologie-scores. Het meest geschikte afkappunt voor de HOST in onbewerkt semen bleek bij $60 \%$ zwelling te liggen. Echter, de bijbehorende lage kappa waarde van 0.151 , betekent dat de overeenstemming tussen de HOST en de IVF resultaten aan het toeval zijn toe te schrijven. Ook de correlatie tussen de zwelling van spermatozoa als gevolg van de hypoosmotische condities en de bevruchting in vitro bleek laag te zijn $(r=0.334)$. MEUSC echter bleek beter te correleren met het resultaat van de in vitro fertilizatie, en bleek ook beter dan de HOST te kunnen voorspellen in welke gevallen geen enkele eicel bevrucht zou worden (Hoofdstuk III).

In hoofdstuk IV worden de resultaten beschreven van een onderzoek verricht bij een grotere en niet-geselecteerde IVF populatie. Het doel van deze studie 
was om te onderzoeken welke parameters van de semenanalyse (inclusief de morfologie volgens de WHO criteria en de MEUSC) betrouwbare selectie criteria zouden zijn voor patiënten die in aanmerking komen voor een IVF behandeling. Als beste afkappunten voor de verschillende klassieke semen parameters werden gevonden: spermatozoa concentratie $20 \mathrm{miljoen} / \mathrm{ml}$, motiliteit $30 \%$, progressieve motiliteit $30 \%$, progressief bewegende spermatozoa concentratie (PMSD) 3 miljoen $/ \mathrm{ml}$, normale morfologie volgens WHO criteria $14 \%$, en normale morfologie volgens strikte criteria $5 \%$. In deze niet-geselecteerde IVF patiëntengroep bleek het beste afkappunt voor de morfologie volgens de WHO criteria beneden het officiële WHO afkappunt uit 1987 van 50\% te liggen. Zeer recent (1993) heeft de WHO dit afkappunt gewijzigd in 30\%. Na de MEUSC bleek de PMSD de beste parameter te zijn om bevruchting in vitro en zwangerschap te kunnen voorspellen. Bij een combinatie van beide parameters beneden hun respectievelijk afkappunt werd een bevruchtingspercentage van slechts $18 \%$ gevonden, en werd geen enkele zwangerschap verkregen. Bij beide parameters boven hun afkappunt werd een bevruchtingspercentage van $72 \%$ en een zwangerschapspercentage van $27 \%$ gevonden.

Voor het onderzoek beschreven in hoofdstuk V werd de morfologie bestudeerd van de spermatozoa die in contact worden gebracht met de eicellen. Deze spermatozoa zijn door middel van een zogenaamde "swim-up" techniek geselecteerd uit het verse semenmonster. De voorspellende waarde ten aanzien van bevruchting in vitro van zowel het percentage, als het aantal spermatozoa met een normale morfologie werd onderzocht. Van alle onderzochte spermaparameters bleek de morfologie de beste correlatie te vertonen tussen de waarden gevonden in het verse semen en in de "swim-up"-oplossing $(r=0.804)$. $\mathrm{Na}$ de swim-up procedure werd een significante stijging gevonden in het percentage morfologisch normale spermatozoa, voor zowel de fertiele patientengroep, als de infertiele groep $(\mathrm{p}<0.001)$. In de fertiele groep werd na de swim-up procedure ook een significante stijging gevonden in het percentage progressief bewegende spermatozoa $(p<0.0005)$, terwijl in de infertiele groep deze stijging niet gevonden werd.

Omdat bij elke patiënt een zelfde aantal progressief bewegende spermatozoa in het inseminatie medium gebracht werden, blijven als mogelijke parameters voor het voorspellen van de IVF resultaten over de totale spermatozoaconcentratie, het percentage spermatozoa met normale morfologie, en het aantal spermatozoa met normale morfologie (IMNS). De totale spermatozoaconcentratie bleek geen effect te hebben op het IVF resultaat. IMNS liet een geringe correlatie zien met de bevruchtingsresultaten $(r=0.336, p<0.001)$, maar gaf geen klinisch acceptabel afkappunt. MEUSC bleek de beste voorspeller (Hoofdstuk IV). Het percentage spermatozoa met een normale morfologie heeft de hoogste voorspellende waarde ten aanzien van de IVF resultaten, zowel in het verse semenmonster als in het swim-up monster. Het zijn ook deze twee parameters die klinisch acceptabele afkappunten geven ( $5 \%$ en $8 \%$, respectievelijk). Bij een 
combinatie uitkomsten ander het afkappunt van zowel de MEUSC van het verse semen, als de MEUSC van het swim-up monster, was het bevruchtingspercentage slechts $7 \%$ en er werd geen enkele zwangerschap gerealiseerd.

Het percentage progressief bewegende spermatozoa kan verhoogd worden met behulp van motiliteitsstimulantia. Pentoxifylline (PF), een fosfodiesterase remmer, wordt vaak gebruikt voor dit doel. In hoofdstuk VI worden de effecten van de incubatie van spermatozoa met PF op de intracellulaire cAMP concentratie en motiliteitsparameters van spermatozoa beschreven. Gevonden werd dat alle motiliteitsparameters significant hoger waren in de PF groep, vergeleken met de controle groep $(\mathrm{p}<0.05)$. In de PF groep werd direct na de incubatie ook een significante verhoging van de intracellulaire cAMP concentratie gevonden $(p<0.0005)$. Als de spermatozoa echter bewerkt werden als voor een IVF behandeling, bleken de intracellulaire cAMP concentraties niet significant verschillend te zijn in de controle en de PF groepen. Deze resultaten zijn in overeenstemming met eerdere bevindingen en laten zien dat, zodra de cAMP niveau's in de spermatozoa eenmaal verhoogd zijn geweest onder invloed van $\mathrm{PF}$, een verdere incubatie van de spermatozoa in medium zonder PF geen invloed meer heeft op de staat van hyperactivatie van de spermatozoa, hoewel de cAMP niveau's weer dalen. PF kan daarom gebruikt worden bij IVF om de progressief bewegende spermatozoaconcentratie te vergroten, en verdere wasprocedures zullen dit stimulerend effect niet te niet doen.

Concluderend kan gesteld worden, dat uit de resultaten beschreven in hoofdstuk II - IV blijkt, dat de morfologische evaluatie van humane spermatozoa, op basis van strikte criteria (MEUSC), een belangrijk instrument is om de bevruchtingspercentages bij in vitro fertilisatie te voorspellen, en dat de MEUSC beter voorspelt dan de morfologische evaluatie op basis van de WHO criteria. Additionele parameters die belangrijk zijn voor de voorspelling van IVF resultaten zijn de PMSD in het verse semenmonster en de MEUSC in het inseminatiemedium. Het feit dat er geen zwangerschappen gevonden werden bij patiënten met MEUSC, PMSD en post swim-up MEUSC scores beneden hun respectievelijke afkappunten, is een motivatie om te zoeken naar behandelprocedures, zoals de incubatie van spermatozoa met PF, die het mogelijk maken om een groter aantal progressief bewegende spermatozoa te verzamelen voor IVF (Hoofdstuk VI). 
Sperm sayısı, hareketliliği ve morfolojisi, ejakülat kalitesini belirleyen faktörler olarak kabul edilmektedir. 1988 yılında Kruger et al. in önerdiği yeni morfoloji değerlendirme tekniği sayesinde, sperm morfolojisi son 5 senedir diğer parametrelerden daha fazla önem kazanmıştır. Aynı dönemlerde, rutin olarak kullanılmakta olan Papanicolau boyama yöntemine alternatif olarak, daha hızlı sonuç veren Diff-Quik boyama yöntemi de önerilmiştir. Preparat kalitesi açısından iki boyama yöntemi arasında fark olmamakla birlikte, Diff-Quik ile boyanmış preparatlarda sperm morfolojisi daha detaylı olarak değerlendirilebilir. Diff-Quik ile boyanmıs preparatlar ayrica daha net bir fona sahiptir.

Sperm morfoloji çalışmaları arasındaki en belirli farklılık değerlendirme yöntemlerinden kaynaklanmaktadır. Sperm morfolojisinin in vitro fertilizasyon (IVF) programlarındaki rolü değerlendirilmiştir. Literatürde ilk kez Dünya Sağlık Örgütü (WHO) ile Kruger tarafindan önerilmiş olan dikkatli morfoloji değerlendirmesi (MEUSC), sperm sayısı ve hareketliliği normal olan hastalarda (IVF programı çerçevesinde) karşılaşıııılmıştır. Hernekadar WHO değerlendirme yöntemi ile in vitro döllenme sonuçları arasında bir korelasyon saptanmışsa da $(r=0.282, p<0.001)$, bu korelasyon dikkatli değerlendirme yöntemi lehinedir $(r=0.555, p<0.000005)($ Chapter II).

Yayınlanmış eşik değerleri kullanmak yerine, yeni değerlendirme yöntemi için, yeni bir eşik değer araştırılmıştır. Normal morfoloji yüzdesi $\% 5$ in altında olan hastaların in vitro döllenme yüzdesi düşmüștür (\%23). Tüm sperm parametreleri için ROC eğrileri oluşturulmuş ve MEUSC en yüksek kappa değeri (0.439) ile birlikte klinikte tek kabul edilebilir parametre olarak saptanmıştır (Chapter II).

Chapter III de hipoosmotik şişme testinin (HOST) klinik yararlılığı değerlendirilmiş ve sperm morfolojisi ile karşılaştırılmıştır. HOST için en uygun eşik değer $\% 60$ olarak şaptanmişsada 0.151 ile sınırlı kalan kappa değeri bu eşik değerin tamamen şans eseri olduğunu vurgulamaktadır. HOST ile in vitro döllenme arasındaki korelasyon da düşüktür $(r=0.334)$. Bunun yanında MEUSC ile in vitro döllenme arasındaki korelasyon yüksek olup, başarısız IVF sonuçlarını (\%0 döllenme) önceden belirleyebilmiştir.

Chapter IV de, hiçbir kısitlamaya gidilmeksizin tüm IVF grubu MEUSC ve WHO değerlendirme yöntemleri çerçevesinde incelenmiştir. Aynı zamanda MEUSC e yardımcı başka parameterelerin olup olmadığını araştırmak amaçlanmıştır. Klasik parametreler için en uygun eşik değerler; sperm sayımı $20 \mathrm{x}$ $10^{6} / \mathrm{ml}$, hareketlilik $\% 30$, progresif hareketlilik $\% 30$, progresif hareketli sperm sayısı (PMSD) $3 \times 10^{6} / \mathrm{ml}$, normal morfolji WHO yöntemi \% 14 , ve normal morfoloji dikkatli değerlendirme yöntemi $\% 5$, olarak saptanmıştır.

Ayırım yapılmamıs bu IVF populasyonunda da WHO yöntemi için saptanmı̧ olan \% 14, Dünya Sağlık Örgütünün o dönemlerde kullandığı \%50 eşik değerin çok altındadır (Dünya Sağlık Örgütü bu değeri yakın zamanda \%30 a 
indirmiștir).

PMSD, MEUSC tan sonra en iyi ikinci parametre olarak saptandığından, bu iki parametre in vitro döllenme ve hamilelik yüzdesi göz önünde tutularak birlikte değerlendirilmiştir. Bu iki parametre, yukarıda verilen eşik değerlerin altına düştüğünde döllenme yüzdesi yalnızca $\% 18$ olup, bu gurupta hiçbir hamilelik saptanmamıștır. Bunun yanında eğer her iki parametre de eşik değerlerinin üzerinde ise döllenme yüzdesi $\% 72$, hamilelik yüzdesi $\% 27$ olarak saptanmıştır. Chapter V de swim-up prosedürü sonrasındaki morfolji ile inseminasyon sıvısı içindeki normal morfolojiye sahip sperm sayısı (IMNS), IVF sonuçları ışı̆̆ında değerlendirilmiştir.

Swim-up öncesi ve sonrası sperm parametreleri değerlendirildiğinde, saptana en yüksek korelasyon morfoloji skorlarına aittir. Bu sonuç swim-up sonrasında saptanan morfoloji yüzdesinin, swim-up öncesinden doğru şekilde tahmin edilebileceğini göstermektedir $(r=0.804)$ (Chapter $V)$.

Tüm gurupta swim-up prosedürü sonrasında progresif motilite yüzdesi ve sayısında, ve normal morfoloji yüzdesinde belirgin bir artış saptanmıştır. Fakat gurup fertil ve infertil olarak ayrı ayrı değerlendirildiğinde progresif hareketlilik yüzdesindeki belirgin artış yalnızca fertil guruba ait olup $(p<0.0005)$, infertil gurupta saptanmamıştır. Normal morfoloji ise her iki gurupta da belirgin artış göstermiştir $(\mathrm{p}<0.001)$.

IVF inseminasyon sıvısında, progresif hareketlilik gösteren sperm sayısı sabitleştirildiğinden, elimizde IVF sonuçlarını önceden belirleyecek parametre olarak yalnızca, bu sıvıdaki sperm sayısı, normal morfoloji yüzdesi ve normal morfolojiye sahip sperm sayısı kalmaktadır. Sperm sayısının IVF sonuçlarına hiçbir etkisi yoktur. IMNS döllenme yüzdeleri ile ılımlı bir korelasyon göstermekle birlikte $(r=0.336, p<0.001)$ klinikte kabul edilebilecek bir eşik değer gösterememektedir. MEUSC ise en belirleyici parametre olma özelliğini korumaktadır (Chapter IV).

IVF sonuçlarını en iyi belirleyici iki parametre swim-up öncesi ve sonrasındaki MEUSC skorlarıdır. Daha önemlisi, klinikte kullanılabilecek eşik değerleri ancak bu iki parametre vermektedir (sırasıyla $\% 5$ ve $\% 8$ ).

$\mathrm{Bu}$ iki parametre birlikte değerlendirildiğinde, eşik değerlerin altında kalan hastalarda döllenme yüzdesi $\% 7$ olup hamilelik saptanmamıştır.

Progresif hareketli sperm sayısı motilite stimülanlarının yardımı ile arttırılabilir. Pentoxifylline (PF), bir fosfodiesteraz inhibitörü olup, geniş bir kullanım alanı bulmuştur.

Chapter VI da, PF inkubasyonunun spermlerdeki intrasellüler siklik adenosinmonofosfat (cAMP) konsantrasyonu ve motilite parametrelrine etkisi incelenmiştir. Tüm motilite parametreleri kontrol gurubu ile karşılaştırıldığında PF gurubunda belirgin olarak yüksektir $(p<0.05)$. Spermatozoa rutin IVF prosedürüne tabi tutulduğunda inrasellüler cAMP konsantrasyonlarında değişiklik saptanamamıștır. Fakat cAMP konsantrasyonları PF inkübasyonunun hemen akabinde ölçüldüğünde, PF gurubu belirgin şekilde yüksektir 
$(\mathrm{p}<0.0005)$.

Bu sonuçlar literatürdekiler ile birlikte değerlendirildiğinde, şu sonuç çıkmaktadır; cAMP konsantrasyonu PF inkübasyonu ile yükselmekle birlikte, PF sıvıdan uzaklaştırıldığında cAMP konsantrasyonu düşse bile bu spermin hiperaktivasyonuna ve spontan akrozom reaksiyonu sensitizasyonuna engel olmamaktadır. Bundan dolayı PF, IVF programlarında uyarıcı olarak kullanılabilir, ayrıca sıvıdan uzaklaştırılması uyarıcı özelliğini etkilememektedir.

Sonuç olarak, Chapter II den IV e kadar olan bölümlerin verileri, dikkatli değerlendirme yönteminin, IVF sonuçları açısından önemli rol oynadığını ve Dünya Sağlık Örgütü yöntemine olan üstünlüğünü vurgulamaktadır.

MEUSC e yardımcı diğer parametreler PMSD ve swim-up sonrası MEUSC dur. Daha önemlisi, bu üç parametreye ait eşik değerlerin altına düşen hastalar klinisyenleri rutin IVF tedavisi dışında, PF incubasyonu gibi daha fazla sayıda progresif hareketli sperm sayısı elde edbilecekleri alternatif tedaviler aramaya motive etmelidir (Chapter VI). 


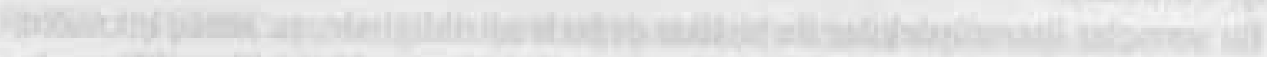

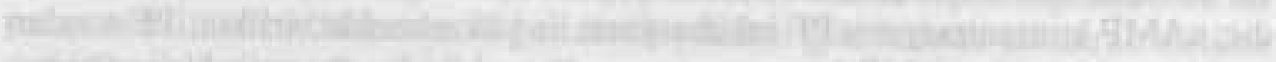

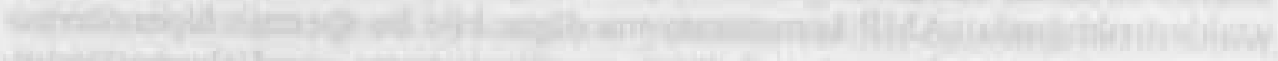

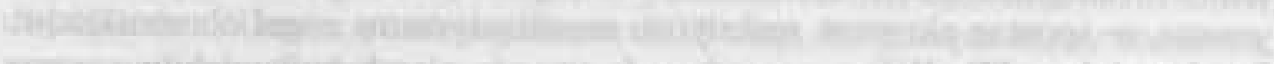

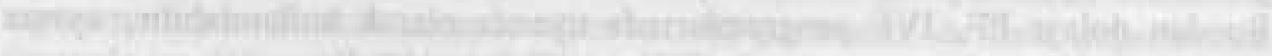

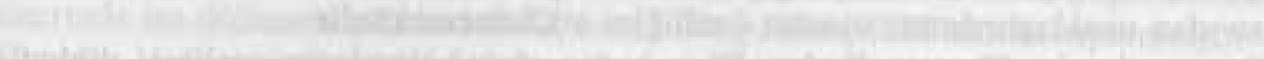

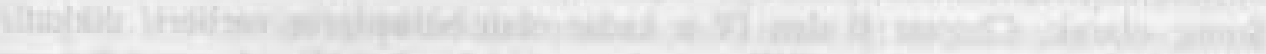

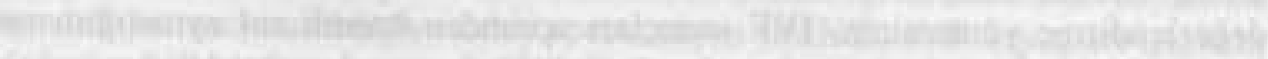
-

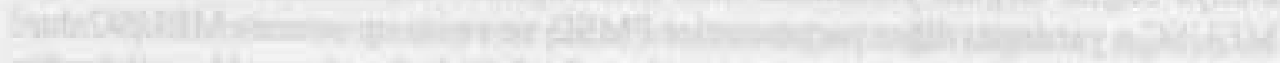

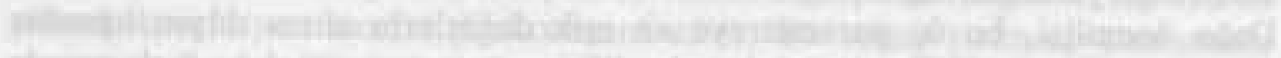

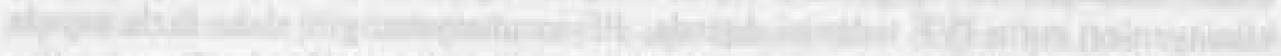

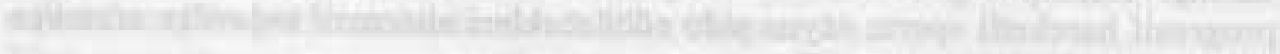

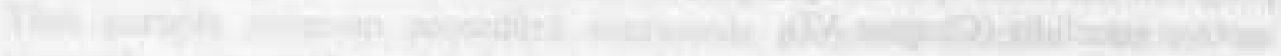

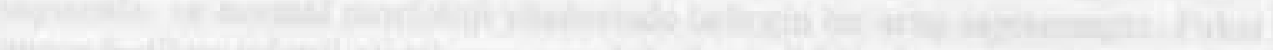

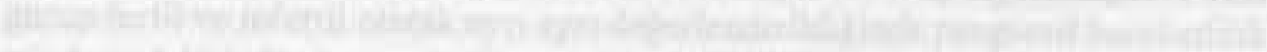

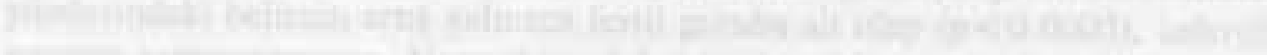

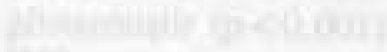

The

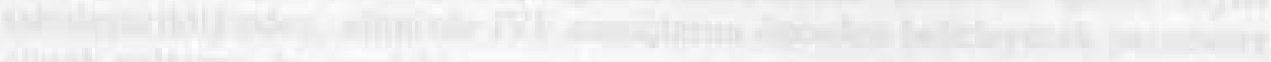

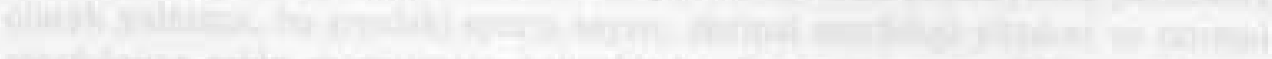

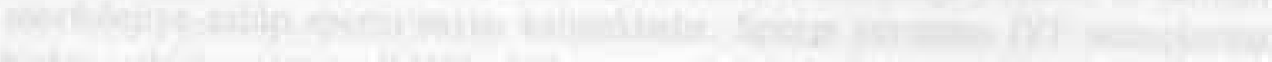

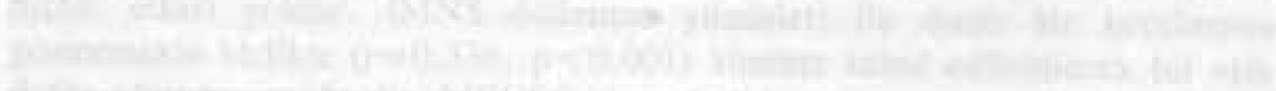

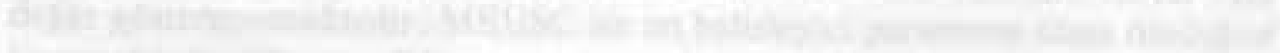

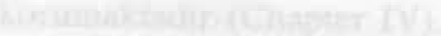

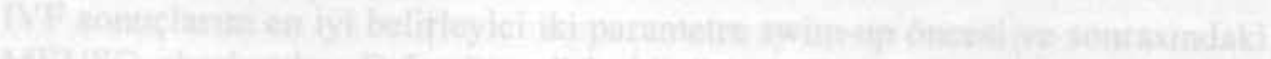

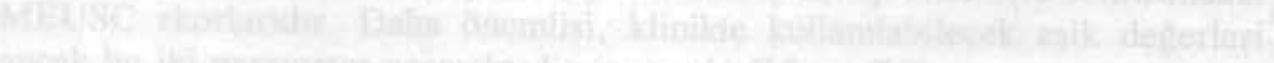

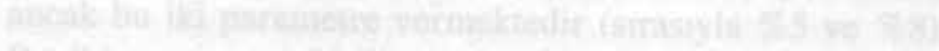

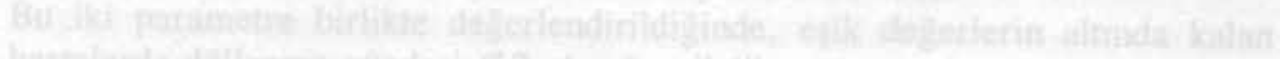

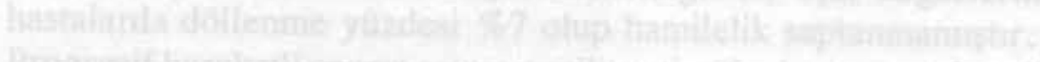

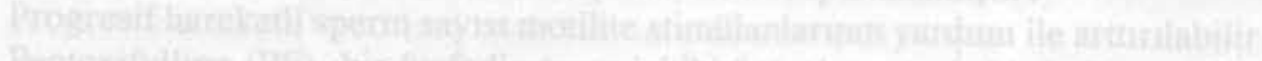

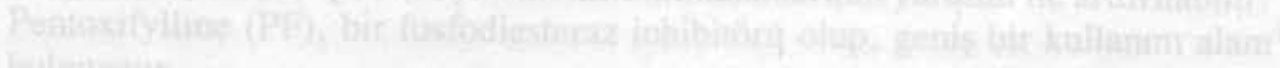

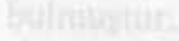

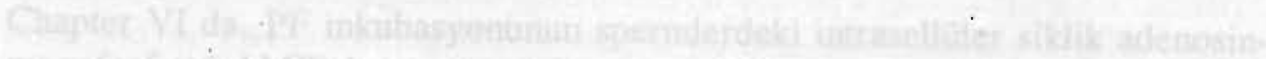

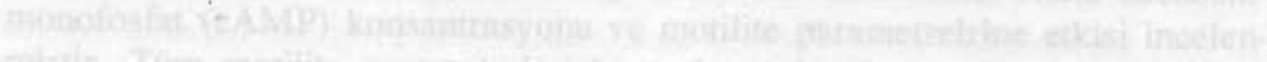

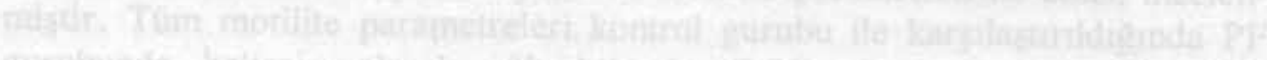

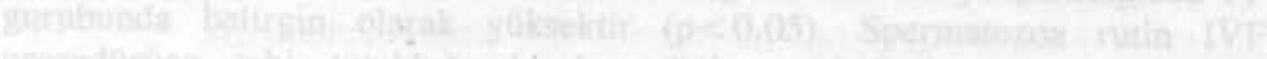

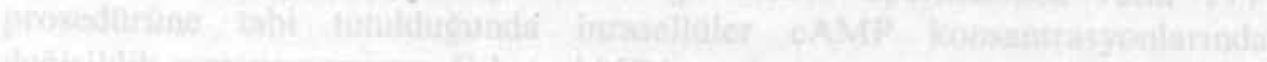

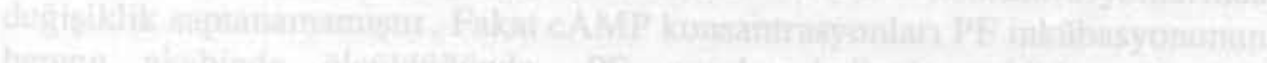

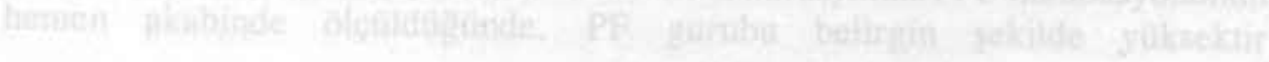




\section{Appendix 1}

There are four possible outcomes of diagnostic tests. 1- True Positive: The patient does have the disease (i.e. is infertile) and the test is positive (abnormal test result, below cut-off point). 2- False Positive: The patient does not have the disease (i.e. is fertile) and the test is positive (abnormal test result, below cutoff point). 3- False Negative: The patient has the disease (i.e. is infertile) and the test is negative (normal test result, above cut-off point). 4- True Negative: The patient does not have the disease (i.e. is fertile) and the test is negative (normal test result, above cut-off point).

\begin{tabular}{||c|c|c||}
\hline \multirow{2}{*}{ TEST } & \multicolumn{2}{|c|}{ DISEASE } \\
\cline { 2 - 3 } & ABSENT & PRESENT \\
\hline POSITIVE & True Positive (a) & (b) False Positive \\
\hline NEGATIVE & False Negative (c) & (d) True Negative \\
\hline
\end{tabular}

The diagnostic properties can be calculated with the following formulas:

$\mathbf{N}=a+b+c+d$

Positive Predictive Value $=a \div(\mathrm{a}+\mathrm{b})$

Negative Predictive Value $=\mathrm{d} \div(\mathrm{c}+\mathrm{d})$

Sensitivity $=a \div(a+c)$

Specificity $=d \div(b+d)$

Likelihood Ratio Positive (LR +$)=$ Sensitivity $\div$ (1-Specificity)

Likelihood Ratio Negative (LR-) $=(1-$ Sensitivity) $\div$ Specificity

Observed Agreement $(\mathrm{OA})=(\mathrm{a}+\mathrm{d}) \div \mathrm{N}$

Agreement by Chance $(A C)=\{[(a+c) \times(a+b) \div N]+[(b+d) \times(c+d) \div N]\} \div N$

Kappa $=(\mathrm{OA}-\mathrm{AC}) \div(1-\mathrm{AC})$

ROC curves are based on sensitivity and specificity. Sensitivity is an index of the ability of the test to detect the disease when it is present, and specificity is the ability of the test to correctly identify the absence of the disease. Sensitivity and specificity can be considered the stable properties of a diagnostic test, because they do not change when different proportions of diseased and well patients are tested. However, they fluctuate mutually when different cut-off points are chosen. The product of this fluctuation reflects the overall usefulness of the test and can be displayed graphically as the ROC curve (Figure 1). 
An ROC curve also displays the implications of using different cut-off points to distinguish between the diseased and non diseased states. The ROC curve for an ideal diagnostic test reaches the upper left-hand corner, whereas a test with no diagnostic value (that is, random) would appear as a 45-degree line through the origin. Therefore, the use of ROC curve analysis is the optimal method to compare different diagnostic tests that are used for the same purpose. The curves of an optimal diagnostic test and a test with no diagnostic value is shown in Figure 1.

Figure 1. Receiver operating characteristic curve.

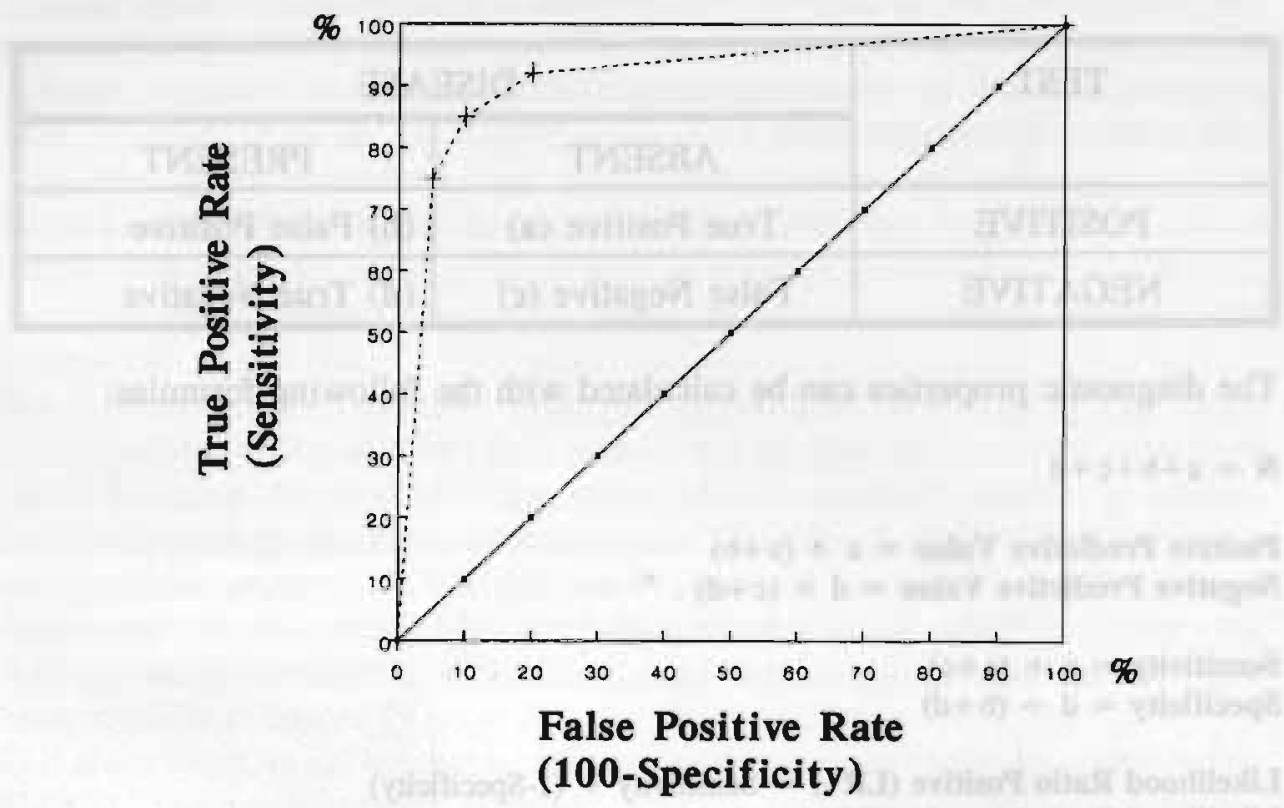

\section{References}

1. Richardson DK, Schwartz JS, Weinbaum PJ, Gabbe SG (1985): Diagnostic tests in obstetrics: a method for improved evaluation. Am J Obs Gyn, 152: 613-8.

2. Peng HQ, Collins JA, Wilson EH, Wrixon W (1987): Receiver-operating characteristics curves for semen analysis variables: methods for evaluating diagnostic tests of male gamete function. Gamete-Res 17: 229-236. 


\section{Appendix 2}

Morphology evaluation using strict criteria (MEUSC):

The guidelines for strict morphology evaluation are $(1,2)$ :

1- The anatomy of the whole spermatozoon should be evaluated.

2- The evaluation should be made in a microscopic field of $\mathrm{x} 1000$.

3- Cell clumps and cell clusters should be ignored.

4- A minimum of 200 total cells in different fields, should be evaluated.

5- The head of the spermatozoon should have a smooth oval configuration with a well defined acrosomal cap.

6- The acrosomal cap should be in the range of $40 \%$ to $70 \%$.

7- Normal spermatoza should have the measurements in Figure 1.

8- The post acrosomal region of the spermatozoon should be evaluated carefully since even a slight abnormality in this region is considered as borderline and borderline forms are considered as abnormal.

9- Normal spermatoza should not have any midpiece or tail abnormalities.

Figure 1. Schematic drawing of a morphologically normal spermatozoon.

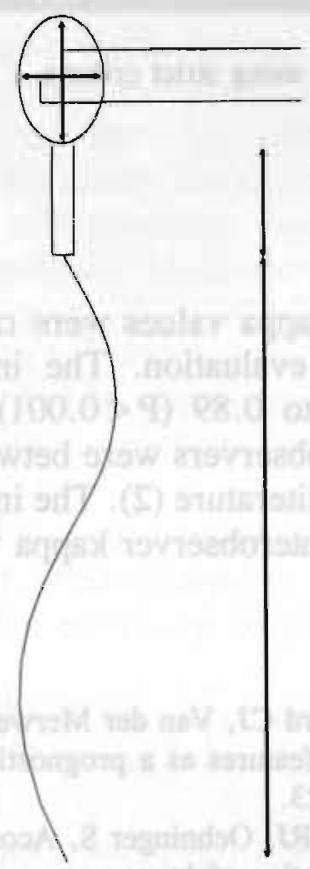

4-5 micrometers

2-3 micrometers

4-5 micrometers

50-55 micrometers
Head

Midpiece 
Figure 2. Photographic illustration of a morphology slide.

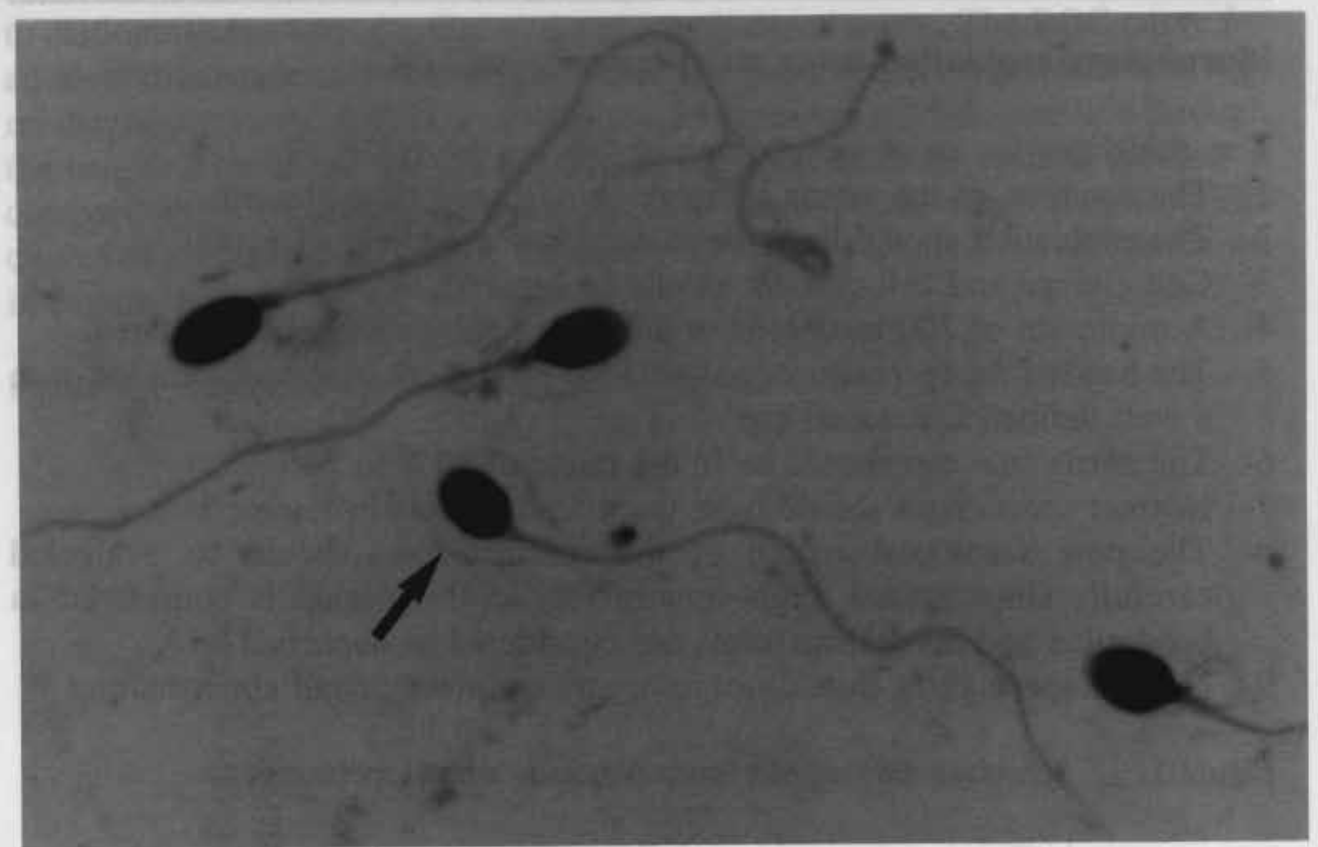

Arrow indicates morphologically normal spermatozoon using strict criteria.

\section{Reliability of morphology evaluation}

Spearman's rank correlation coefficients and kappa values were calculated to determine the reliability of the morphology evaluation. The intraobserver correlation coefficiencies were between 0.68 to $0.89(\mathrm{P}<0.001)$ and interobserver correlation coefficients between four observers were between 0.58 to $0.74(\mathrm{P}<0.001)$, which is comparable with the literature $(2)$. The intraobserver kappa values were between 0.75 to 0.86 , and interobserver kappa values were 0.62 to 0.81 .

\section{References}

1. Kruger TF, Menkveld R, Stander FSH, Lombard CJ, Van der. Merwe JP, Van Zyl JA, Smith KA (1986): Sperm morphologic features as a prognostic factor in in vitro fertilization. Fertil Steril, 46: 1118-1123.

2. Menkveld R, Kruger TF, Oettle EE, Swanson RJ, Oehninger S, Acosta A (1991): Basic principals and practical aspects. In Atlas of human sperm morphology. Menkveld R, Oettle EE, Kruger TF, Swanson RJ, Acosta A, Oehninger S (eds), Williams and Wilkins, Baltimore, Maryland, USA, pg 1-6. 


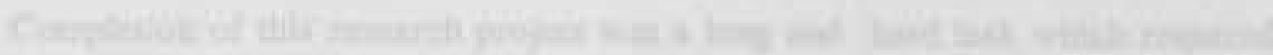

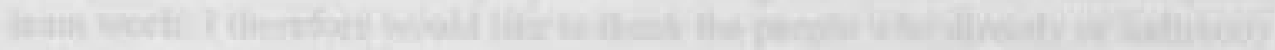

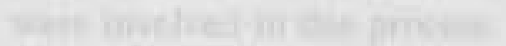

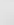

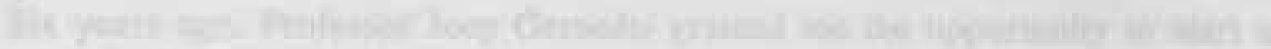

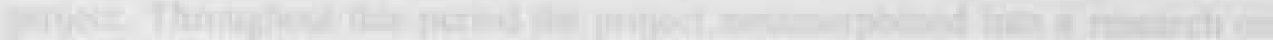

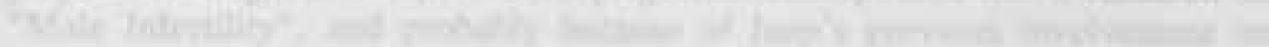

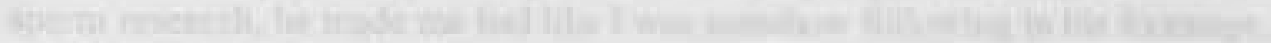

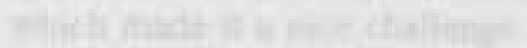

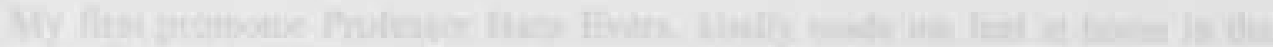

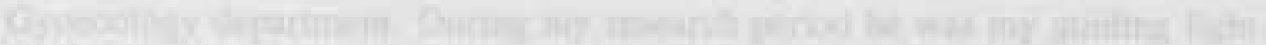

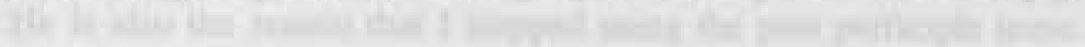

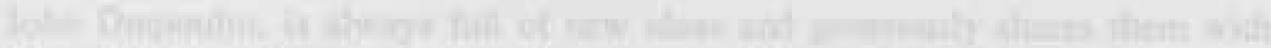
H:

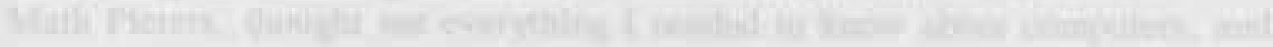

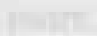

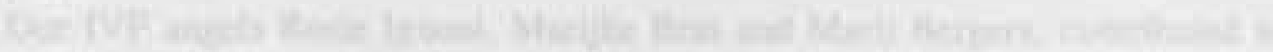

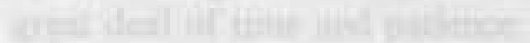

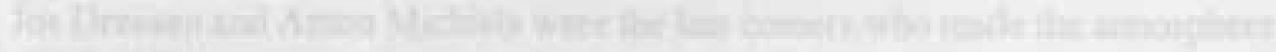

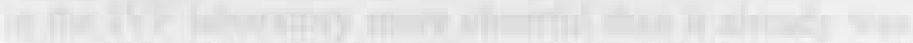

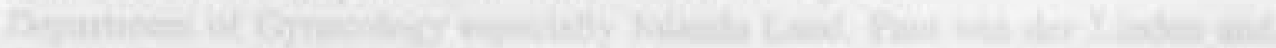

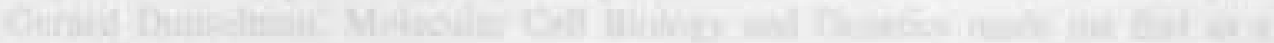

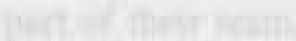




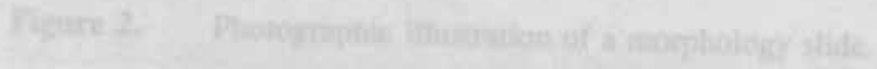

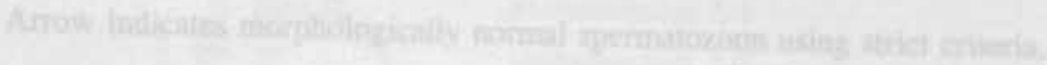

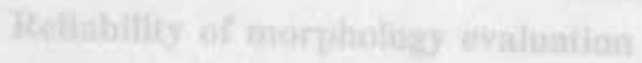

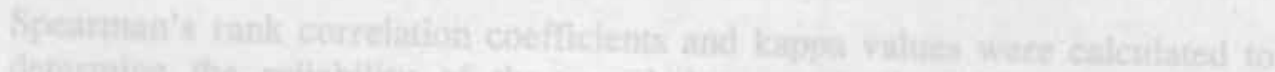

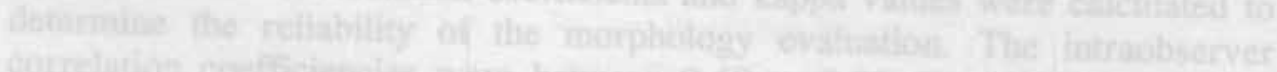

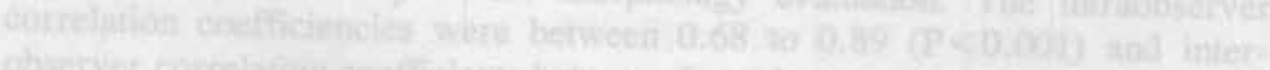

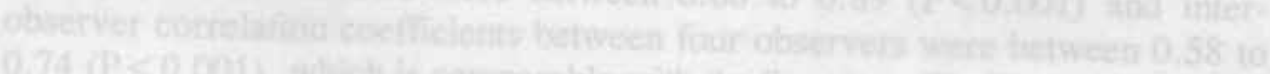

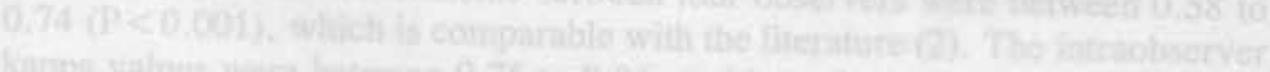

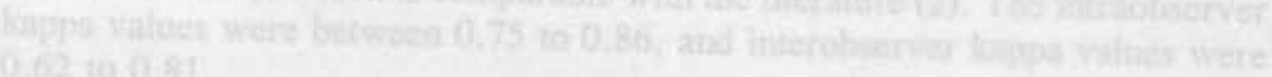
0.02100081

\section{Replenente}

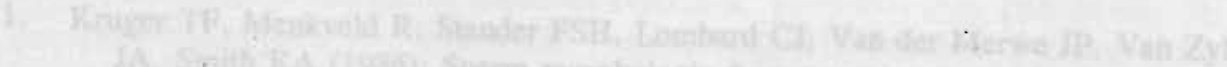

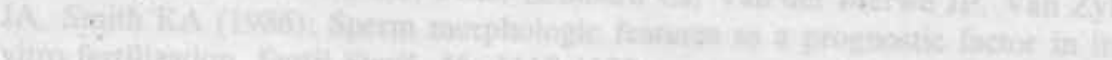

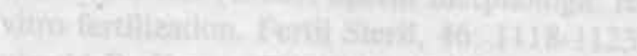

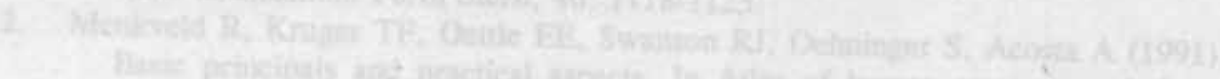

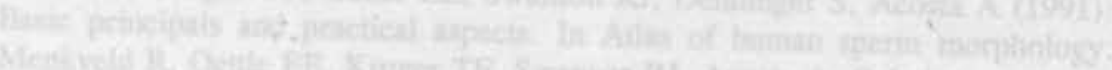

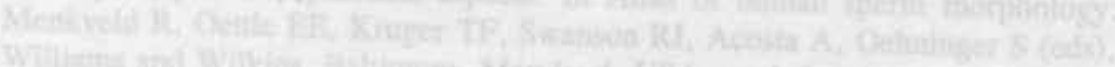

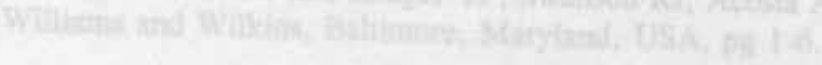




\section{Thank You}

Completion of this research project was a long and hard task which required team work. I therefore would like to thank the people who directly or indirectly were involved in this process.

Six years ago, Professor Joep Geraedts granted me the opportunity to start a project. Throughout this period the project metamorphosed into a research on "Male Infertility", and probably because of Joep's previous involvement on sperm research, he made me feel like I was somehow following in his footsteps, which made it a nice challenge.

My first promotor Professor Hans Evers, kindly made me feel at home in the Gynecology department. During my research period he was my guiding light. $\mathrm{He}$ is also the reason that I stopped using the past participle tense.

John Dumoulin, is always full of new ideas and generously shares them with his colleagues. Learning to conduct a research from him was beneficial.

Math Pieters, thought me everything I needed to know about computers, and more.

Our IVF angels Rosie Ignoul, Marijke Bras and Marij Bergers, contributed a great deal of time and patience.

Jos Dreesen and Anton Michiels were the late comers who made the atmosphere in the IVF laboratory more cheerful than it already was.

Department of Gynecology especially Jolanda Land, Paul van der Linden and Gerard Dunselman, Molecular Cell Biology and Genetics made me feel as a part of their team.

Francis v.d. Lubbe always tried her best and in some magical way succeeded in making pictures perfect. I will always cherish her enthusiasm.

I learned a lot about biochemistry during my involvement with Department of Biochemistry. Paul Menheere, Sylvia Beckers-Jäckle, José Vranken and Jos Wiersma were extremely helpful.

With the help of the personnel from the library, especially Helene Branges, Monique de Haes and Guus Foppen, I was sure to get the required literature on time.

The personnel from AVD, ICA and CPV were very cooperative and helpful.

The Degree Committee kindly spared their precious time and made the finalization possible. 
I also would like to mention the friendship and care that I received from Betty and King Bochardt during this process.

Writing this part of the thesis was the biggest challenge of all. It required a lot of soul searching. I wanted to find the correct words to express my gratitude. No citation was proper. Finally I decided to thank all of you sincerely. It was a wonderful experience and Maastricht will always have a special place in my life. 


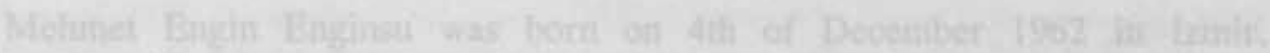

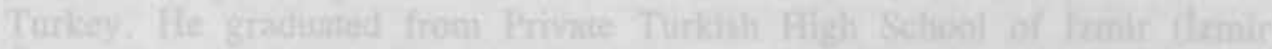

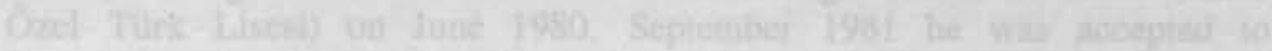

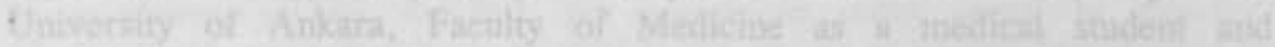

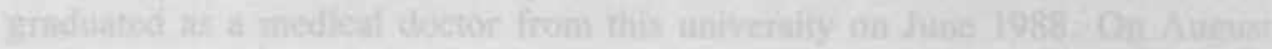

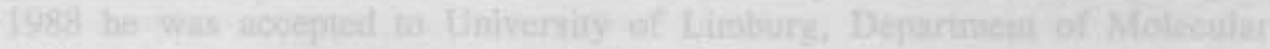

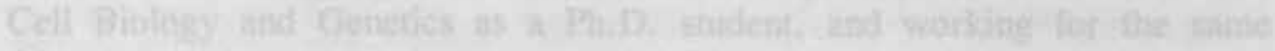

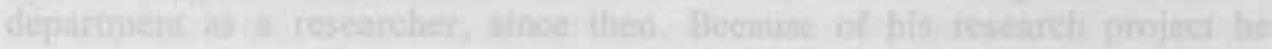

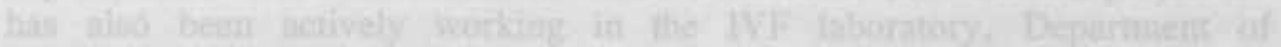

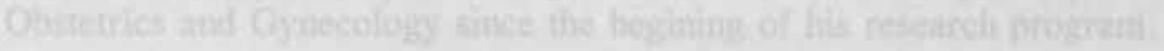




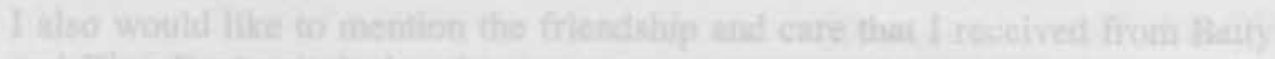

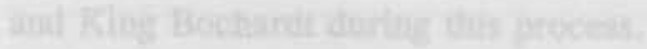

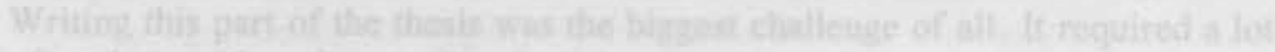

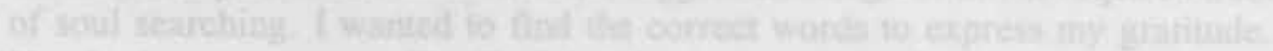

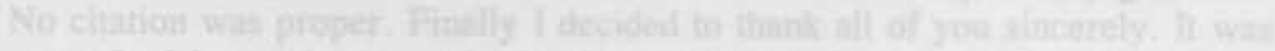

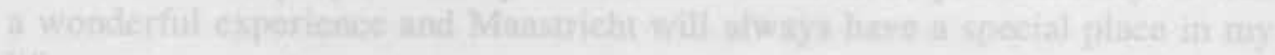
Jifie 


\section{Curriculum Vitae}

Mehmet Engin Enginsu was born on 4th of December 1962 in İzmir, Turkey. He graduated from Private Turkish High School of İzmir (İzmir Özel Türk Lisesi) on June 1980. September 1981 he was accepted to University of Ankara, Faculty of Medicine as a medical student and graduated as a medical doctor from this university on June 1988. On August 1988 he was accepted to University of Limburg, Department of Molecular Cell Biology and Genetics as a Ph.D. student, and working for the same department as a researcher, since then. Because of his research project he has also been actively working in the IVF laboratory, Department of Obstetrics and Gynecology since the begining of his research program. 


\section{Abstracts}

Enginsu ME, Dumoulin JCM, Pieters MHEC, Bras M, Evers JLH, Geraedts JPM: Evaluation of human sperm morphology with Diff-Quik staining: correlation of morphology with fertilization rate in vitro. Reinier de Graaf Symposium, Maastricht; 30 may-2 june 1990.

Dumoulin JCM, Bras M, Land JL, Pieters MHEC, Enginsu ME, Evers JL: A high number of inseminating sperm in human in vitro fertilization is detrimental for the subsequent embryonic development in vitro. Human Reproduction Abstracts from 7th. meeting of ESHRE.

Bras M, Dumoulin JCM, Land JL, Pieters MHEC, Enginsu ME, Evers JL: Effect of the number of inseminating sperm in human in vitro fertilization on the subsequent embryonic development in vitro. 7th ESHRE congress for para-medical's, Paris: 1991

Geraedts JPM, Pieters MHEC, Dumoulin JCM, Menheere PPCA, Land JA, Enginsu ME, Evers JLH. Meiotic immaturity in two sisters. European Society of Human Genetics 24th Annual Meeting, 1992 Copenhagen.

Enginsu ME, Dumoulin JCM, Pieters MHEC, Bras M, Ignoul RCM, Land JA, Evers JLH, Geraedts JPM. The role of male factor in fertility prediction in vitro. International Symposium on Preimplantation Genetics and Assisted Fertilization. 1992, Brussels.

Enginsu ME, Dumoulin JCM, Pieters MHEC, Bras M, Ignoul RCM, Land JL, Evers JL, Geraedts JPM: Strict criteria sperm morphology predicts fertilization in vitro. Human Reproduction 1992: Abstracts from 8th. meeting of ESHRE.

Pieters MHEC, Dumoulin JCM, Menheere PPCA, Land JL, Enginsu ME, Evers JL, Geraedts JPM: Non-germinal vesicle breakdown (NGVB) as a familial cause of infertility. Human Reproduction 1992; Abstracts from 8th. meeting of ESHRE.

Evers JLH, Enginsu ME, Dumoulin JCM, Pieters MHEC, Bras M, Ignoul RCM, Land JL, Evers JL, Geraedts JPM: Strict criteria sperm morphology and IVF result. Journal of Assisted Reproduction and Fertility 1993 (Suppl.); Abstracts of the $8^{\text {th }}$ World Congress on In Vitro Fertilization and Alternate Assisted Reproduction, Kyoto, Japan.

Enginsu ME, Dumoulin JCM, Evers JLH, Geraedts JPM: Morphology evaluation using strict criteria (MEUSC) and IVF failure. Submitted.

Enginsu ME, Menheere PPCA, Dumoulin JCM, Evers JLH, Geraedts JPM: Pentoxifylline and sperm intracellular cAMP concentration. Submitted.

Enginsu ME. Dumoulin JCM, Evers JLH, Geraedts JPM: Strict sperm morphology and IVF failure. Submitted. 


\section{Articles}

Enginsu ME, Dumoulin JCM, Pieters MHEC, Bras M, Evers JLH, Geraedts JPM: Evaluation of human sperm morphology using strict criteria after Diff-Quik staining: correlation of morphology with fertilization in vitro. Human Reprod 1991, Vol 6: 854858

Enginsu ME, Dumoulin JCM, Pieters MHEC, Bergers M, Evers JLH, Geraedts JPM: Comparison between the hypoosmotic swelling test and morphology evaluation using strict criteria in predicting fertilization in vitro. Journal of Assisted Reproduction and Genetics 1992 Vol 9: 259-264

Enginsu ME, Dumoulin JCM, Land JA, Evers JLH, Geraedts JPM: Male factor as determinant of in vitro fertilization outcome. Human Reproduction 1992 7(8): 1136-1140.

Dumoulin JCM, Bras M, Land JL, Pieters MHEC, Enginsu ME, Geraedts JPM, Evers JL: Effect of the number of inseminating sperm in human in vitro fertilization on the subsequent embryonic development in vitro. Human Reproduction 1992 7(7): 1010-1013.

Enginsu ME, Dumoulin JCM, Pieters MHEC, Evers JLH, Geraedts JPM: Predictive value of morphologically normal sperm concentration in the medium for in vitro fertilization. International Journal of Andrology 1993 16(2): 113-120.

Dumoulin JCM, Bergers-Janssen JM, Bras M, Pieters MHEC, Enginsu ME, Geraedts JPM, Evers JLH: The protective effects of polymers on the cryopreservation of human and mouse zonae pellucidae and embryos. Submitted to Fertility and Sterility for publication.

Enginsu ME, Menheere PPCA, Evers JLH, Geraedts JPM: The effect of Pentoxifylline on intracellular sperm cAMP concentration. Submitted for publication.

Enginsu ME: Efficiency and predictive value of different methods of morphological evaluation. In: Diagnosis and therapy of male factor in assisted reproduction. Parthenon Publishing. In print. 
